Pacific

Journal of

Mathematics

SEMICLASSICAL DIFFERENTIAL STRUCTURES

EDWIN J. BEgGS AND SHAHN MAJID 


\title{
SEMICLASSICAL DIFFERENTIAL STRUCTURES
}

\author{
EDWIN J. BEGGS AND SHAHN MAJID
}

\begin{abstract}
We semiclassicalise the standard notion of differential calculus in noncommutative geometry on algebras and quantum groups. We show in the symplectic case that the infinitesimal data for a differential calculus is a symplectic connection, and interpret its curvature as lowest order nonassociativity of the exterior algebra. Semiclassicalisation of the noncommutative torus provides an example with zero curvature. In the Poisson-Lie group case we study left-covariant infinitesimal data in terms of partially defined preconnections. We show that the moduli space of bicovariant infinitesimal data for quasitriangular Poisson-Lie groups has a canonical reference point which is flat in the triangular case. Using a theorem of Kostant, we completely determine the moduli space when the Lie algebra is simple: the canonical preconnection is the unique point other than in the case of $\mathfrak{s l}_{n}$, $n>2$, when the moduli space is 1-dimensional. We relate the canonical preconnection to Drinfeld twists and thereby quantise it to a super coquasiHopf exterior algebra. We also discuss links with Fedosov quantisation.
\end{abstract}

\section{Introduction}

Usually the quantisation problem in physics consists of a commutative algebra of functions equipped with a Poisson bracket and the search for a noncommutative algebra with commutators reproducing this to lowest order in a deformation parameter $\hbar$. It is well known that actually the converse problem is more well posed: given a noncommutative algebra which is a flat deformation one may semiclassicalise its structure and recover the Poisson bracket of which it is a quantisation. Either way Poisson brackets are the semiclassical data for associative noncommutative algebras.

In this second point of view the starting point is the noncommutative algebra itself. In the last two decades the 'geometry' of such algebras has been well developed in different approaches, such as from operator theory [Connes 1994] or

MSC2000: 58B32, 58B34, 81R50.

Keywords: Poisson geometry, symplectic connection, noncommutative geometry, quantum group, differential calculus, nonassociative algebra.

Majid is a University Research Fellow of the Royal Society. 
Hopf algebras [Majid 1995], and in particular the notion of differential structures, quantum bundles, Riemannian structures etc have been fairly well established from an intrinsically noncommutative algebraic point of view. In this paper and its sequel we will semiclassicalise these various notions from noncommutative geometry to elucidate the classical infinitesimal data of which they are quantisations. In this first part we limit ourselves to the differential calculus and differential forms. In a sequel we will proceed to quantum bundles and Riemannian structures at the semiclassical level.

Let $A$ be an algebra. The by-now standard notion of differential calculus in noncommutative geometry is to specify an $A$ - $A$-bimodule $\Omega^{1}$ of '1-forms' and a linear map d : $A \rightarrow \Omega^{1}$ obeying:

(1) Leibniz rule: $\mathrm{d}(a b)=a(\mathrm{~d} b)+(\mathrm{d} a) b$ for all $a, b \in A$.

(2) Surjectivity: $\{a \mathrm{~d} b\}$ span $\Omega^{1}$.

We do not demand that $a \mathrm{~d} b=(\mathrm{d} b) a$, i.e., that 1-forms and functions commute, just as we do not demand that $A$ be commutative. The above is a first order calculus and we will find that its semiclassical data is a compatible partially defined 'preconnection' (a symplectic torsion free connection in the symplectic case when the symplectic form is central to lowest order), see Propositions 2.1 and 3.3.

We can ask further for an entire differential graded 'algebra' of forms of all degree and $\mathrm{d}$ such that $\mathrm{d}^{2}=0$. Usually one demands an associative such exterior algebra and this case corresponds to a flat symplectic connection or preconnection. The formal analysis for both results is in Section 2 and the geometric meaning is in Section 3. We find in particular three super-Jacobi identity obstructions $J_{1}, J_{2}, J_{3}$. The geometric meaning of the $J_{1}$ obstruction turns out to be the curvature of the preconnection. We give geometric conditions for the others also (involving now the torsion). An example of a flat connection is provided by the noncommutative torus at the semiclassical level.

Next, in Section 4, we specialise to the case where $A$ is a Hopf algebra or 'quantum group'. These provide examples of noncommutative geometry which are well controlled though the requirements of the 'group' structure. Their semiclassicalisation was worked out in the 1980 s by V. G. Drinfeld as the notion of a Poisson-Lie group. In this context it is natural to restrict to differential structures that are left, right or bicovariant [Woronowicz 1989]. We analyse the left covariance restrictions on a Poisson-Lie group $G$ at the semiclassical level in Sections 4A and 4B and give a formulation of the data in Lie algebraic terms of a map $\Xi: \mathfrak{g} \rightarrow \mathfrak{g} \otimes \mathfrak{g}$. We give an example on the group manifold $\mathrm{SU}_{2}$ in Section $4 \mathrm{C}$. After that, Section $4 \mathrm{D}$ analyzes the stronger requirement of a bicovariant calculus at the semiclassical level, where Proposition 4.16 together with Corollary 4.7 reduce the classification to Ad-invariant symmetric maps $\hat{\boldsymbol{\Xi}}: \mathfrak{g} \rightarrow \operatorname{Sym}^{2}(\mathfrak{g})$ when the Poisson-Lie group is 
quasitriangular (this includes the standard $q$-deformation quantum groups at the semiclassical level). We conclude in this case (Theorem 4.18 and Proposition 4.19) that there is a canonical choice and it has curvature given by the Schouten bracket $\llbracket r_{-}, r_{-} \rrbracket$, where $r_{-}$is the antisymmetric part of the quasitriangular structure $r \in \mathfrak{g} \otimes \mathfrak{g}$. Thus the classical Yang-Baxter equation has a direct geometric meaning as curvature. We show (Theorem 4.20) that for all simple $\mathfrak{g}$ other than the $\mathfrak{s l}_{n}$ series, $n>2$, it is the only choice for a first order bicovariant semiclassical calculus (i.e., a compatible preconnection). Moreover, our results imply that this extends associatively to lowest order if and only if $\mathfrak{g}$ is triangular. This fits with the 'quantum Lie functor' for a canonical differential calculus on quasitriangular Hopf algebras introduced in [Gomez and Majid 2003], which is known to typically give trivial answers unless the quantum group is triangular. Our results prove indeed that a strictly quasitriangular Poisson-Lie group has no bicovariant calculus that is a deformation (with the same dimensions etc.) of the classical calculus in a high degree of generality. This explains the typical experience in quantum group theory where strictly quasitriangular quantum groups typically do not admit bicovariant differential calculi of the usual classical dimension but require some form of central or other extension. What is remarkable is that this obstruction identified in our analysis is not at the semiclassical first order level where we find the perfectly good canonical preconnection above; it enters at the semiclassical super-Jacobi identity level concerning the associativity in the bimodule and exterior algebra structures.

This last point is taken up in Section 5, where we provide a different point of view on the canonical preconnection for the standard quasitriangular Poisson-Lie groups, now inspired by Drinfeld's theory of quasi-Hopf algebras [1987]. Drinfeld showed (effectively) that the standard quantum groups $C_{\hbar}(G)$ in a suitable deformation setting are isomorphic to the twisting by a cochain of a coquasiHopf algebra structure on the classical group function algebras. Even though the cochain is not a cocycle it happens that the resulting algebra is associative. This accident does not extend to the exterior algebra, i.e., when the classical exterior algebra is similarly twisted as in [Majid and Oeckl 1999] it becomes nonassociative. We semiclassicalise this construction and understand our canonical preconnection in these terms as corresponding to a quasiassociative calculus (Proposition 5.3). Conversely put, we quantise the canonical preconnection to a super coquasi-Hopf exterior algebra $\Omega\left(C_{\hbar}(G)\right)$ by these means.

Finally, we note that our main result that the data for a semiclassical calculus is a pair consisting of a Poisson bracket and a compatible connection or preconnection is, in the symplectic case, precisely the data used by Fedosov [1996] to solve the quantisation problem. We make some remarks about this in Section 6. Our result suggests that in the Fedosov construction there is also for free a noncommutative differential calculus also constructed from the input data and with semiclassical 
limit the Fedosov symplectic connection. If so then whereas the problem of quantising a symplectic structure to an algebra is not unique as one must chose the connection, quantising the pair consisting of a symplectic structure and connection to an algebra and differential calculus would be unique. Also, because of the role played in Hamiltonian mechanics by symplectic structures, we expect a physical role also of the symplectic connection and here its interpretation as controlling the quantisation of the differential structure suggests a possible role.

In this paper we use the following conventions for the torsion and curvature tensors. For vector fields $U, V$ and $W$ we have

$$
\begin{aligned}
T(U, V)^{k} & =T_{i j}^{k} U^{i} V^{j}, & T(U, V) & =\nabla_{U} V-\nabla_{V} U-[U, V], \\
R(U, V)(W)^{l} & =R_{i j k}^{l} W^{i} U^{j} V^{k}, & R(U, V)(W) & =\nabla_{U} \nabla_{V} W-\nabla_{V} \nabla_{U} W-\nabla_{[U, V]} W .
\end{aligned}
$$

In terms of Christoffel symbols we have

$$
\begin{aligned}
\left(\nabla_{V} W\right)^{l} & =V^{k}\left(W_{, k}^{l}+\Gamma_{k i}^{l} W^{i}\right), \quad T_{i j}^{k}=\Gamma_{i j}^{k}-\Gamma_{j i}^{k}, \\
R_{i j k}^{l} & =\frac{\partial \Gamma_{k i}^{l}}{\partial x^{j}}-\frac{\partial \Gamma_{j i}^{l}}{\partial x^{k}}+\Gamma_{k i}^{m} \Gamma_{j m}^{l}-\Gamma_{j i}^{m} \Gamma_{k m}^{l} .
\end{aligned}
$$

Note added in press: In fact the requirement of flatness in the usual associative case has been found earlier in [Hawkins 2004], using a different notion of 'contravariant connections'; this does not affect our main results, which are intended to reach beyond the flat case. We thank E. Hawkins for bringing this work to our attention and for helpful comments.

\section{Deformation analysis of noncommutative differential structures}

In this section we perform the required algebraic deformation analysis and prove some basic lemmas. Their geometric meaning will then be explored in later sections.

As usual, we deform commutative multiplication on $C^{\infty}(M)$ on a classical manifold $M$ to the associative multiplication $x \bullet y$, where $x \bullet y=x y+O(\hbar)$. If we assume that the commutator can be written as $[x, y]_{\bullet}=x \bullet y-y \bullet x=\hbar\{x, y\}+$ $O\left(\hbar^{2}\right)$, we see that $\{x, y\}=-\{y, x\}$ and (by considering the two ways of writing $z \bullet x \bullet y-x \bullet y \bullet z$ to first order in $\hbar),\{z, x y\}=\{z, x\} y+x\{z, y\}$, that is, $\{$,$\} is a Pois-$ son bracket. Formally speaking, the deformed algebra $C_{\hbar}^{\infty}(M)$ can be formulated as an algebra over $\mathbb{C}[\hbar]$, topologically free with $C_{\hbar}^{\infty}(M) / \hbar C_{\hbar}^{\infty}(M) \cong C^{\infty}(M)$. However, for most of the paper we actually need only that structure maps depend on an $\hbar$ parameter permitting of power series expansion and comparison of lower order terms as specified, which is therefore the line that we take. The same remark applies for differential forms in what follows. 
2A. Deformation of the classical bimodule structure. In this section we begin with assumptions on the associativity of the differential calculus, and see what this means in terms of the supercommutators $[$,$] . . We define a formal deformation of$ the differential calculus on $M$ to lowest order in $\hbar$ to be the following:

As sets the functions $C^{\infty}(M)$ and $n$-forms $\Omega^{n}(M)$ take their classical values. The symbol $\bullet$ is used for the deformed multiplication. $\Omega^{1}(M)$ is a bimodule over $\left(C^{\infty}(M), \bullet\right)$ to order $O\left(\hbar^{2}\right)$, i.e., for $x, y \in C^{\infty}(M)$ and $\tau \in \Omega^{1}(M)$

$$
\begin{aligned}
& (x \bullet y) \bullet \tau-x \bullet(y \bullet \tau)=O\left(\hbar^{2}\right), \\
& \tau \bullet(x \bullet y)-(\tau \bullet x) \bullet y=O\left(\hbar^{2}\right), \\
& (x \bullet \tau) \bullet y-x \bullet(\tau \bullet y)=O\left(\hbar^{2}\right) .
\end{aligned}
$$

We also suppose that $x \bullet \tau=x \tau+O(\hbar)$ and $\tau \bullet x=x \tau+O(\hbar)$, and hence define $\gamma$ by

$$
x \bullet \tau-\tau \bullet x=[x, \tau]_{\bullet}=\hbar \gamma(x, \tau)+O\left(\hbar^{2}\right) .
$$

We make the assumption that the deformed exterior multiplication $\wedge_{\bullet}$ is associative to $O\left(\hbar^{2}\right)$. Note that where one of the forms is a zero-degree form, we just use $\bullet$ instead of $\wedge_{\bullet}$. We also assume that $\tau \wedge_{\bullet} \eta=\tau \wedge \eta+O(\hbar)$.

The deformed d operator $\mathrm{d}^{\bullet}: \Omega^{n}(M) \rightarrow \Omega^{n+1}(M)$, is related to the usual $\mathrm{d}$ by $\mathrm{d}^{\bullet} x=\mathrm{d} x+O(\hbar)$. Also $\mathrm{d}^{\bullet}$ is a graded derivation to order $O\left(\hbar^{2}\right)$, i.e.,

$$
\mathrm{d}^{\bullet}\left(\xi \wedge_{\bullet} \eta\right)=\mathrm{d}^{\bullet} \xi \wedge_{\bullet} \eta+(-1)^{\operatorname{deg} \xi} \xi \wedge_{\bullet} \mathrm{d}^{\bullet} \eta+O\left(\hbar^{2}\right) .
$$

Proposition 2.1. The map $\gamma(-, \tau)$ is a derivation on $C^{\infty}(M)$ for all $\tau \in \Omega^{1}(M)$ and obeys

$$
\gamma(x, \tau y)=\gamma(x, \tau) y+\tau\{x, y\} .
$$

We call any map $\gamma$ with these properties a preconnection. Moreover, $\gamma$ is compatible with the Poisson structure in the sense

$$
\mathrm{d}\{x, y\}=\gamma(x, \mathrm{~d} y)-\gamma(y, \mathrm{~d} x) .
$$

Proof. From the approximate bimodule rules (2-1) we have, for $x, y \in C^{\infty}(M)$ and $\tau \in \Omega^{1}(M)$.

$$
[x \bullet y, \tau]_{\bullet}=x \bullet[y, \tau]_{\bullet}+[x, \tau]_{\bullet} \bullet y+O\left(\hbar^{2}\right),
$$

from which we deduce

$$
\gamma(x y, \tau)=x \gamma(y, \tau)+\gamma(x, \tau) y .
$$

The next formula is deduced from

$$
[x, \tau \bullet y]_{\bullet}=[x, \tau]_{\bullet} \bullet y+\tau \bullet[x, y]_{\bullet}+O\left(\hbar^{2}\right),
$$


and the last formula from the approximate derivation rule (2-3)

$$
\mathrm{d}^{\bullet}[x, y]_{\bullet}=\left[\mathrm{d}^{\bullet} x, y\right]_{\bullet}+\left[x, \mathrm{~d}^{\bullet} y\right]_{\bullet}+O\left(\hbar^{2}\right) .
$$

The terminology for $\gamma$ is justified in Section 3 below.

Proposition 2.2. The commutator of a function $x$ with higher forms is given by the following formula, which can also be viewed as the extension of $\gamma$ to higher forms:

$$
[x, \tau \wedge \bullet \eta]_{\bullet}=\hbar \gamma(x, \tau \wedge \eta)+O\left(\hbar^{2}\right)=\hbar(\gamma(x, \tau) \wedge \eta+\tau \wedge \gamma(x, \eta))+O\left(\hbar^{2}\right) .
$$

Proof. This can be seen by rearranging the formula

$$
\left[x, \tau \wedge_{\bullet} \eta\right]_{\bullet}=x \bullet\left(\tau \wedge_{\bullet} \eta\right)-\left(\tau \wedge_{\bullet} \eta\right) \bullet x+O\left(\hbar^{2}\right) .
$$

2B. Supercommutator of forms and Jacobi terms. If the operation $\wedge_{\bullet}$ on $\Omega^{*}$ is associative (up to $O\left(\hbar^{2}\right)$ ) and supercommutative (up to $O(\hbar)$ ), then the superJacobi identities for the supercommutator [, ]. hold up to $O\left(\hbar^{2}\right)$. We now ask what conditions would be necessary for the super-Jacobi identities to hold up to $O\left(\hbar^{3}\right)$.

Definition 2.3. Define $J_{i}: C^{\infty}(M)^{\otimes 3} \rightarrow \Omega^{i}(M)$ by

$$
\begin{aligned}
& {\left[x,[y, \mathrm{~d} z]_{\bullet}\right]_{\bullet}+\left[\mathrm{d} z,[x, y]_{\bullet}\right]_{\bullet}+\left[y,[\mathrm{~d} z, x]_{\bullet}\right]_{\bullet}=\hbar^{2} J_{1}(x, y, z)+O\left(\hbar^{3}\right),} \\
& {\left[x,[\mathrm{~d} y, \mathrm{~d} z]_{\bullet}\right]_{\bullet}-\left[\mathrm{d} z,[x, \mathrm{~d} y]_{\bullet}\right]_{\bullet}+\left[\mathrm{d} y,[\mathrm{~d} z, x]_{\bullet}\right]_{\bullet}=\hbar^{2} J_{2}(x, y, z)+O\left(\hbar^{3}\right),} \\
& {\left[\mathrm{d} x,[\mathrm{~d} y, \mathrm{~d} z]_{\bullet}\right]_{\bullet}+\left[\mathrm{d} z,[\mathrm{~d} x, \mathrm{~d} y]_{\bullet}\right]_{\bullet}+\left[\mathrm{d} y,[\mathrm{~d} z, \mathrm{~d} x]_{\bullet}\right]_{\bullet}=\hbar^{2} J_{3}(x, y, z)+O\left(\hbar^{3}\right) .}
\end{aligned}
$$

Proposition 2.4. (1) If $\mathrm{d} J_{2}$ vanishes identically then so does $J_{3}$.

(2) If $\mathrm{d} J_{1}$ vanishes identically then $J_{2}$ is totally symmetric in its 3 arguments.

Proof. By applying $\mathrm{d}^{\bullet}$ we see that $\mathrm{d} J_{2}(x, y, z)=J_{3}(x, y, z)+O(\hbar)$, so the vanishing of $\mathrm{d} J_{2}$ implies the vanishing of $J_{3}$. For the second statement, note that $\mathrm{d} J_{1}(x, y, z)=J_{2}(x, y, z)-J_{2}(y, z, x)+O(\hbar)$, and by combining this with the more obvious identity $J_{2}(x, z, y)=J_{2}(x, y, z)$ we find that the vanishing of $\mathrm{d} J_{1}$ implies that $J_{2}(x, y, z)$ is totally symmetric in $x, y$ and $z$.

Proposition 2.5. Suppose that the first super-Jacobi identity (for two functions and a 1-form) holds to $O\left(\hbar^{2}\right)$, that $J_{2}(x, x, x)=0$ for all $x \in C^{\infty}(M)$, and that the following conditions are satisfied for all $x, y \in C^{\infty}(M), \tau, \eta \in \Omega^{1}$ and $\pi \in \Omega^{2}$ :

$$
\begin{aligned}
{[\tau, x \eta]_{\bullet} } & =[\tau, x]_{\bullet} \wedge \eta+x[\tau, \eta]_{\bullet}+O\left(\hbar^{2}\right), \\
{[y, x \pi]_{\bullet} } & =[y, x]_{\bullet} \pi+x[y, \pi]_{\bullet}+O\left(\hbar^{2}\right), \\
{[y, \tau \wedge \eta]_{\bullet} } & =[y, \tau]_{\bullet} \wedge \eta+\tau \wedge[y, \eta]_{\bullet}+O\left(\hbar^{2}\right) .
\end{aligned}
$$

Then the second super-Jacobi identity (for a function and two 1-forms) holds to $O\left(\hbar^{2}\right)$. 
Proof. As the super-Jacobi identity for two functions and a 1-form holds to $O\left(\hbar^{2}\right)$, we see that $J_{1}$ vanishes identically. By Proposition $2.4 J_{2}$ is completely symmetric, and as $J_{2}(x, x, x)=0$ for all $x \in C^{\infty}(M)$ we deduce that $J_{2}$ is identically zero. Now use the fact that linear combinations of the form $x \mathrm{~d} y$ span $\Omega^{1}$.

Proposition 2.6. Suppose that the conditions for Proposition 2.5 hold, and that the following conditions hold for all $x \in C^{\infty}(M), \tau, \eta, \xi \in \Omega^{1}$ and $\pi \in \Omega^{2}$ :

$$
\begin{aligned}
{[\tau, \xi \wedge \eta]_{\bullet} } & =[\tau, \xi]_{\bullet} \wedge \eta-\xi \wedge[\tau, \eta]_{\bullet}+O\left(\hbar^{2}\right), \\
{[\tau, x \pi]_{\bullet} } & =[\tau, x]_{\bullet} \wedge \pi+\tau \wedge[y, \pi]_{\bullet}+O\left(\hbar^{2}\right), \\
{[x \tau, \pi]_{\bullet} } & =[x, \pi]_{\bullet} \wedge \tau+x[\tau, \pi]_{\bullet}+O\left(\hbar^{2}\right) .
\end{aligned}
$$

Then the third super-Jacobi identity (for three 1-forms) holds to $O\left(\hbar^{2}\right)$.

Proof. Use the fact that linear combinations of the form $x d y$ are dense in $\Omega^{1}$.

\section{Geometric interpretation of the semiclassical data}

Here we look at the geometric meaning of the map $\gamma$ in the semiclassical data. The full picture emerges in the symplectic case as a symplectic connection, but first some remarks about the general case. For general Poisson bracket we have only a preconnection or 'partially defined connection' which we denote $\hat{\nabla}_{x}$. It should be thought of and sometimes is a usual covariant derivative $\nabla_{\hat{x}}$ along Hamiltonian vector fields $\hat{x}=\{x,-\}$ associated to $x \in C^{\infty}(M)$, and is defined by

$$
\hat{\nabla}_{x} \tau=\gamma(x, \tau) \text { for all } \tau \in \Omega^{1}(M) .
$$

Indeed, the derivation property of a connection on Hamiltonian vector fields is $\hat{\nabla}_{x}(y \tau)=y \hat{\nabla}_{x} \tau+\hat{x}(y) \tau$, which is (2-4) in Proposition 2.1. Meanwhile, writing $\{x y, z\}=y\{x, z\}+x\{y, z\}$ for all $z$ as $\widehat{x y}=y \hat{x}+x \hat{y}$, the derivation property of $\gamma(-, \tau)$ plays the role of the usual tensoriality of a connection with respect to the vector field direction of differentiation.

Similarly, we define the curvature of a preconnection in the usual way but only, morally speaking, on such vector fields, where

$$
R(x, y)=\hat{\nabla}_{x} \hat{\nabla}_{y}-\hat{\nabla}_{y} \hat{\nabla}_{x}-\hat{\nabla}_{\{x, y\}}
$$

given that the Jacobi identity for the Poisson bracket means that $[\hat{x}, \hat{y}]=\widehat{\{x, y\}}$. Its deformation-theoretic meaning is:

Proposition 3.1. For a semiclassical differential calculus,

$$
\left[x,[y, \tau]_{\bullet}\right]_{\bullet}+\left[\tau,[x, y]_{\bullet}\right]_{\bullet}+\left[y,[\tau, x]_{\bullet}\right]_{\bullet}=\hbar^{2} R(x, y)(\tau)+O\left(\hbar^{3}\right) ;
$$

that is, the obstruction to the first super-Jacobi identity is $J_{1}(x, y, z)=R(x, y)(\mathrm{d} z)$. 
Proof. First we calculate

$$
\left[x,[y, \tau]_{\bullet}\right]_{\bullet}=\left[x, \hbar \hat{\nabla}_{y} \tau+O\left(\hbar^{2}\right)\right]_{\bullet}=\hbar^{2} \hat{\nabla}_{x} \hat{\nabla}_{y} \tau+O\left(\hbar^{3}\right) .
$$

Applying this into the first super-Jacobi identity with two functions $x, y$ and a 1form $\tau$ and the definition of $\gamma$ gives the result.

In the same way, we define the torsion tensor again as usual but partially, by

$$
T(x, y)=\hat{\nabla}_{x} \hat{y}-\hat{\nabla}_{y} \hat{x}-\widehat{\{x, y\}}
$$

where $\hat{\nabla}_{x}$ on vector fields is defined as usual via

$$
\left\langle\hat{\nabla}_{x} v, \tau\right\rangle=\hat{x}(\langle v, \tau\rangle)-\left\langle v, \hat{\nabla}_{x} \tau\right\rangle
$$

for any vector field $v$ and all 1-forms $\tau$.

Proposition 3.2. For a compatible preconnection in the sense of (2-4)-(2-5), the torsion obeys

$$
T(x, y)(\mathrm{d} z)+\text { cyclic }=0 \quad \text { for all } x, y, z \in C^{\infty}(M) .
$$

Proof. From the definitions, we have

$$
\langle T(x, y), \mathrm{d} z\rangle=\left\langle\hat{x}, \hat{\nabla}_{y} \mathrm{~d} z\right\rangle-\left\langle\hat{y}, \hat{\nabla}_{x} \mathrm{~d} z\right\rangle
$$

for all $x, y, z \in C^{\infty}(M)$, using the definition of torsion, converting to an operation on forms and using the Jacobi identity for the Poisson bracket. We then take the cyclic sum $x \rightarrow y \rightarrow z \rightarrow x$, use (2-5) three times, and the Jacobi identity again.

This completes our general comments. For further explicit computations, we will suppose that $M$ is finite-dimensional with coordinate patch with coordinate functions $\left(x^{1}, \ldots, x^{n}\right)$. We use the summation convention for repeated indices. Suppose that the Poisson structure is given by

$$
\{y, z\}=\omega^{i j} \frac{\partial y}{\partial x^{i}} \frac{\partial z}{\partial x^{j}},
$$

where $\omega^{i j}=-\omega^{j i}$ and $y, z$ are functions. The Jacobi identity for $\{$,$\} gives$

$$
\omega^{i s} \frac{\partial \omega^{j k}}{\partial x^{s}}+\omega^{j s} \frac{\partial \omega^{k i}}{\partial x^{s}}+\omega^{k s} \frac{\partial \omega^{i j}}{\partial x^{s}}=0 .
$$

From (2-6) we can write

$$
\gamma\left(y, \mathrm{~d} x^{i}\right)=c_{n}^{i k} \frac{\partial y}{\partial x^{k}} \mathrm{~d} x^{n},
$$

or alternatively $\gamma\left(x^{k}, \mathrm{~d} x^{i}\right)=c_{n}{ }^{i k} \mathrm{~d} x^{n}$. Then from (2-4) we get

$$
\gamma\left(y, a_{i} \mathrm{~d} x^{i}\right)=\left(\omega^{k q} \frac{\partial a_{n}}{\partial x^{q}}+c_{n}{ }^{i k} a_{i}\right) \frac{\partial y}{\partial x^{k}} \mathrm{~d} x^{n} .
$$


From (2-5) we get

$$
\frac{\partial \omega^{i j}}{\partial x^{n}}=c_{n}{ }^{j i}-c_{n}{ }^{i j} .
$$

Given a function $y$ the associated Hamiltonian vector field $\hat{y}$ is of course defined by

$$
\hat{y}=\omega^{k q} \frac{\partial y}{\partial x^{k}} \frac{\partial}{\partial x^{q}} .
$$

3A. The symplectic case. To simplify the computations we now specialise to the nondegenerate case, where the matrix $\omega^{i j}$ is invertible. We write its inverse as $\omega_{i j}$, so that $\omega^{i j} \omega_{j k}=\delta_{k}^{i}$. Then equation (3-4) shows that the 2-form $\omega_{i j} \mathrm{~d} x^{i} \wedge \mathrm{d} x^{j}$ is closed, and so is a symplectic form. We now use material from [Gelfand et al. 1998]. We can rewrite the formula for $\gamma$ as

$$
\gamma(y, \tau)=\omega^{k q} \frac{\partial y}{\partial x^{k}} \nabla_{q}(\tau),
$$

where $\nabla$ is now a fully defined covariant connection,

$$
\nabla_{q}\left(a_{n} \mathrm{~d} x^{n}\right)=\left(\frac{\partial a_{n}}{\partial x^{q}}+\omega_{q s} c_{n}{ }^{i s} a_{i}\right) \mathrm{d} x^{n}=\left(\frac{\partial a_{n}}{\partial x^{q}}-\Gamma_{q n}^{i} a_{i}\right) \mathrm{d} x^{n} .
$$

The formula for the Christoffel symbols is

$$
\Gamma_{q n}^{i}=-\omega_{q s} c_{n}^{i s} \quad \text { or } \quad c_{n}^{i k}=-\omega^{k q} \Gamma_{q n}^{i} .
$$

From (3-5) we see that

$$
\frac{\partial \omega^{i j}}{\partial x^{n}}+\omega^{i q} \Gamma_{q n}^{j}+\omega^{q j} \Gamma_{q n}^{i}=0
$$

which can be rewritten using the torsion tensor $T_{q n}^{j}=\Gamma_{q n}^{j}-\Gamma_{n q}^{j}$ as

$$
\nabla_{n} \omega^{i j}+\omega^{i q} T_{q n}^{j}+\omega^{q j} T_{q n}^{i}=0
$$

Proposition 3.3. The 2-form $\omega$ commutes in the $\bullet$ product with all functions to $O\left(\hbar^{2}\right)$ if and only if the connection preserves $\omega$ and is torsion free.

Proof. We use Darboux coordinates where $\omega_{i j}$ is constant. If the 2 -form $\omega$ is central, then we see that the connection preserves $\omega$, i.e.,

$$
\omega^{i q} \Gamma_{n q}^{j}+\omega^{q j} \Gamma_{n q}^{i}=0
$$

From (3-6) we see that

$$
\omega^{i q} \Gamma_{q n}^{j}+\omega^{q j} \Gamma_{q n}^{i}=0
$$

As in [Gelfand et al. 1998] we set $\Gamma_{i k j}=\omega_{i l} \Gamma_{k j}^{l}$, and then the last two equations give $\Gamma_{s n r}=\Gamma_{r n s}$ and $\Gamma_{s r n}=\Gamma_{r s n}$, so $\Gamma_{s n r}$ is totally symmetric and the torsion vanishes. 


\section{B. The second Jacobi identity in the symplectic case.}

Lemma 3.4. For a 1-form $\tau$ and a vector field $Y$,

$$
\mathrm{d}\left(\nabla_{Y} \tau\right)-\nabla_{Y} \mathrm{~d} \tau=\left(\left(T_{j l}^{n} Y^{j}+Y_{; l}^{n}\right) \tau_{i ; n}-N_{j i l}^{k} \tau_{k} Y^{j}\right) \mathrm{d} x^{l} \wedge \mathrm{d} x^{i},
$$

where

$$
N_{j i l}^{k}=T_{j i ; l}^{k}+\frac{1}{2} R_{j l i}^{k}+T_{j l}^{n} T_{i n}^{k}+\frac{1}{2} T_{l i}^{n} T_{j n}^{k} .
$$

Proof.

$$
\begin{aligned}
\mathrm{d}\left(\nabla_{Y} \tau\right)-\nabla_{Y} \mathrm{~d} \tau & =\left(\left(\Gamma_{j l}^{n} \tau_{i, n}-\Gamma_{j i, l}^{k} \tau_{k}\right) Y^{j}+\left(\tau_{i, j}-\Gamma_{j i}^{k} \tau_{k}\right) Y_{, l}^{j}\right) \mathrm{d} x^{l} \wedge \mathrm{d} x^{i} \\
& =\left(\left(\Gamma_{j l}^{n} \tau_{i, n}-T_{j i, l}^{k} \tau_{k}-\Gamma_{i j, l}^{k} \tau_{k}\right) Y^{j}+\tau_{i, j} Y_{, l}^{j}\right) \mathrm{d} x^{l} \wedge \mathrm{d} x^{i} .
\end{aligned}
$$

Now we use

$$
\begin{aligned}
R_{j l i}^{k} \mathrm{~d} x^{l} \wedge \mathrm{d} x^{i} & =\left(\Gamma_{i j, l}^{k}-\Gamma_{l j, i}^{k}+\Gamma_{i j}^{m} \Gamma_{l m}^{k}-\Gamma_{l j}^{m} \Gamma_{i m}^{k}\right) \mathrm{d} x^{l} \wedge \mathrm{d} x^{i} \\
& =2\left(\Gamma_{i j, l}^{k}+\Gamma_{i j}^{m} \Gamma_{l m}^{k}\right) \mathrm{d} x^{l} \wedge \mathrm{d} x^{i}
\end{aligned}
$$

to get

$$
\begin{aligned}
& \mathrm{d}\left(\nabla_{Y} \tau\right)-\nabla_{Y} \mathrm{~d} \tau\left(\left(\Gamma_{j l}^{n} \tau_{i, n}-T_{j i, l}^{k} \tau_{k}-\frac{1}{2} R_{j l i}^{k} \tau_{k}+\Gamma_{i j}^{n} \Gamma_{l n}^{k} \tau_{k}\right) Y^{j}+\tau_{i ; j} Y_{, l}^{j}\right) \mathrm{d} x^{l} \wedge \mathrm{d} x^{i} \\
&=\left(\left(\Gamma_{j l}^{n} \tau_{i, n}-T_{j i, l}^{k} \tau_{k}-\frac{1}{2} R_{j l i}^{k} \tau_{k}+\Gamma_{i j}^{n} \Gamma_{l n}^{k} \tau_{k}-\Gamma_{l j}^{n}\left(\nabla_{n} \tau_{i}\right)\right) Y^{j}\right. \\
&\left.+\left(\nabla_{j} \tau_{i}\right)\left(\nabla_{l} Y^{j}\right)\right) \mathrm{d} x^{l} \wedge \mathrm{d} x^{i} \\
&=\left(\left(T_{j l}^{n} \tau_{i ; n}-T_{j i, l}^{k} \tau_{k}-\frac{1}{2} R_{j l i}^{k} \tau_{k}+\Gamma_{i j}^{n} \Gamma_{l n}^{k} \tau_{k}+\Gamma_{j l}^{n} \Gamma_{n i}^{k} \tau_{k}\right) Y^{j}\right. \\
&\left.+\left(\nabla_{j} \tau_{i}\right)\left(\nabla_{l} Y^{j}\right)\right) \mathrm{d} x^{l} \wedge \mathrm{d} x^{i} \\
&=\left(\left(T_{j l}^{n} \tau_{i ; n}-T_{j i ; l}^{k} \tau_{k}-\frac{1}{2} R_{j l i}^{k} \tau_{k}+\Gamma_{j i}^{n} \Gamma_{l n}^{k} \tau_{k}+\Gamma_{j l}^{n} \Gamma_{n i}^{k} \tau_{k}\right.\right. \\
&\left.\left.-\Gamma_{l j}^{n} T_{n i}^{k} \tau_{k}-\Gamma_{l i}^{n} T_{j n}^{k} \tau_{k}\right) Y^{j}+\left(\nabla_{j} \tau_{i}\right)\left(\nabla_{l} Y^{j}\right)\right) \mathrm{d} x^{l} \wedge \mathrm{d} x^{i} \\
&=\left(\left(T_{j l}^{n} \tau_{i ; n}-T_{j i ; l}^{k} \tau_{k}-\frac{1}{2} R^{k}{ }_{j l i} \tau_{k}-T_{j l}^{n} T_{i n}^{k} \tau_{k}-\frac{1}{2} T_{l i}^{n} T_{j n}^{k} \tau_{k}\right) Y^{j}\right. \\
&=\left(\left(T_{j l}^{n} Y^{j}+Y_{;}^{n}\right) \tau_{i ; n}-N_{j i l}^{k} \tau_{k} Y^{j}\right) \mathrm{d} x^{l} \wedge \mathrm{d} x^{i} .
\end{aligned}
$$

Proposition 3.5. The supercommutator between two 1-forms $\tau$ and $\xi$ is

$$
[\xi, \tau]_{\bullet}=\hbar \omega^{j n} \nabla_{j} \xi \wedge \nabla_{n} \tau+\hbar \omega^{j n} N_{j i l}^{k} \xi_{n} \tau_{k} \mathrm{~d} x^{l} \wedge \mathrm{d} x^{i}+O\left(\hbar^{2}\right) .
$$

By the symmetry of $[\xi, \tau]$ • we must have $\omega^{j n} N_{j i l}^{k}-\omega^{j n} N_{j l i}^{k}$ symmetric in $n k$.

Proof. It is enough to consider $\xi=a \mathrm{~d} b$. Begin with

$$
\begin{aligned}
{[\mathrm{d} b, \tau]_{\bullet} } & =\mathrm{d}[b, \tau]_{\bullet}-[b, \mathrm{~d} \tau]_{\bullet}+O\left(\hbar^{2}\right) \\
& =\hbar\left(\mathrm{d} \nabla_{\hat{b}}-\nabla_{\hat{b}} \mathrm{~d}\right) \tau+O\left(\hbar^{2}\right) \\
& =\hbar\left(\left(T_{j l}^{n} \hat{b}^{j}+\hat{b}_{; l}^{n}\right) \tau_{i ; n}-N_{j i l}^{k} \tau_{k} \hat{b}^{j}\right) \mathrm{d} x^{l} \wedge \mathrm{d} x^{i}+O\left(\hbar^{2}\right) .
\end{aligned}
$$


Now we use $\hat{b}^{n}=\omega^{i n} b_{, i}$ to get

$[\mathrm{d} b, \tau]_{\bullet}=\hbar\left(\left(T_{j l}^{n} \omega^{m j} b_{, m}+\omega_{; l}^{m n} b_{, m}+\omega^{m n}\left(b_{, m}\right)_{; l}\right) \tau_{i ; n}\right.$

$$
\left.-N_{j i l}^{k} \tau_{k} \omega^{m j} b_{, m}\right) \mathrm{d} x^{l} \wedge \mathrm{d} x^{i}+O\left(\hbar^{2}\right)
$$

Using equation (3-7) for the covariant derivative of $\omega$,

$$
\begin{aligned}
{[\mathrm{d} b, \tau] \bullet } & =\hbar\left(\left(T_{j l}^{m} \omega^{n j} b_{, m}+\omega^{m n}\left(b_{, m}\right)_{; l}\right) \tau_{i ; n}-N_{j i l}^{k} \tau_{k} \omega^{m j} b_{, m}\right) \mathrm{d} x^{l} \wedge \mathrm{d} x^{i}+O\left(\hbar^{2}\right) \\
& =\hbar\left(\left(T_{j l}^{m} \omega^{n j} b_{, m}+\omega^{j n}(b, j)_{; l}\right) \tau_{i ; n}-N_{j i l}^{k} \tau_{k} \omega^{n j} b_{, n}\right) \mathrm{d} x^{l} \wedge \mathrm{d} x^{i}+O\left(\hbar^{2}\right) \\
& =\hbar \omega^{n j}\left(\left(T_{j l}^{m} b_{, m}-\left(b_{, j}\right)_{; l}\right) \tau_{i ; n}-N_{j i l}^{k} \tau_{k} b_{, n}\right) \mathrm{d} x^{l} \wedge \mathrm{d} x^{i}+O\left(\hbar^{2}\right) \\
& =\hbar \omega^{n j}\left(-\left(b_{, l}\right)_{; j} \tau_{i ; n}-N_{j i l}^{k} \tau_{k} b_{, n}\right) \mathrm{d} x^{l} \wedge \mathrm{d} x^{i}+O\left(\hbar^{2}\right) .
\end{aligned}
$$

Now using the next equation gives the answer;

$$
[a \mathrm{~d} b, \tau]_{\bullet}=a[\mathrm{~d} b, \tau]_{\bullet}+\mathrm{d} b \wedge[a, \tau]_{\bullet}+O\left(\hbar^{2}\right) .
$$

Proposition 3.6. If the curvature vanishes and $E^{n k}{ }_{l i} \equiv \omega^{j n} N_{j i l}^{k}$ is covariantly constant, then the obstruction $J_{2}$ to the second super-Jacobi identity vanishes.

Proof. First consider the case $E=0$. In Darboux coordinates where $\omega$ is constant,

$$
\begin{aligned}
{\left[a,[\xi, \tau]_{\bullet}\right]_{\bullet}-\left[\tau,[a, \xi]_{\bullet}\right]_{\bullet}+\left[\xi,[\tau, a]_{\bullet}\right]_{\bullet} } \\
=\hbar\left(\left[a, \omega^{j n} \nabla_{j} \xi \wedge \nabla_{n} \tau\right]_{\bullet}-\left[\tau, \nabla_{\hat{a}} \xi\right]_{\bullet}-\left[\xi, \nabla_{\hat{a}} \tau\right]_{\bullet}\right)+O\left(\hbar^{3}\right) \\
\quad=\hbar^{2}\left(\omega^{j n}\left[\nabla_{\hat{a}}, \nabla_{j}\right] \xi \wedge \nabla_{n} \tau+\omega^{j n} \nabla_{j} \xi \wedge\left[\nabla_{\hat{a}}, \nabla_{n}\right] \tau\right)+O\left(\hbar^{3}\right) .
\end{aligned}
$$

If the curvature is zero this becomes

$$
\begin{aligned}
\hbar^{2}\left(\omega^{j n} \nabla_{\{\hat{a}, j\}} \xi \wedge \nabla_{n} \tau+\right. & \left.\omega^{j n} \nabla_{j} \xi \wedge \nabla_{\{\hat{a}, n\}} \tau\right)+O\left(\hbar^{3}\right) \\
& =\hbar^{2}\left(\omega^{m n} \nabla_{\{\hat{a}, m\}} \xi \wedge \nabla_{n} \tau+\omega^{j m} \nabla_{j} \xi \wedge \nabla_{\{\hat{a}, m\}} \tau\right)+O\left(\hbar^{3}\right) \\
& =\hbar^{2}\left(\omega^{m n}\left\{\hat{a}, \partial_{m}\right\}^{j}+\omega^{j m}\left\{\hat{a}, \partial_{m}\right\}^{n}\right) \nabla_{j} \xi \wedge \nabla_{n} \tau+O\left(\hbar^{3}\right) .
\end{aligned}
$$

Now we calculate

$$
\begin{aligned}
\omega^{m n}\left\{\hat{a}, \partial_{m}\right\}^{j} & =-\omega^{m n}\left(\hat{a}^{j}\right)_{, m}=\omega^{n m} \omega^{i j} a_{, i m}=\omega^{n m} \omega^{i j} a_{, m i} \\
& =\omega^{n i} \omega^{m j} a_{, i m}=-\omega^{j m} \omega^{n i} a_{, i m}=-\omega^{j m}\left\{\hat{a}, \partial_{m}\right\}^{n} .
\end{aligned}
$$

Now we consider the general case, where

$$
\begin{aligned}
& {\left[a,[\xi, \tau]_{\bullet}\right]_{\bullet}-\left[\tau,[a, \xi]_{\bullet}\right]_{\bullet}+\left[\xi,[\tau, a]_{\bullet}\right]_{\bullet}} \\
& =\hbar\left[a, E^{n k}{ }_{l i} \xi_{n} \tau_{k} \mathrm{~d} x^{l} \wedge \mathrm{d} x^{i}\right]_{\bullet}-E^{n k}{ }_{l i} \tau_{n}\left(\nabla_{\hat{a}} \xi\right)_{k} \mathrm{~d} x^{l} \wedge \mathrm{d} x^{i} \\
& -E^{n k}{ }_{l i} \xi_{n}\left(\nabla_{\hat{a}} \tau\right)_{k} \mathrm{~d} x^{l} \wedge \mathrm{d} x^{i}+O\left(\hbar^{3}\right),
\end{aligned}
$$

which leads to $\nabla_{\hat{a}} E_{l i}^{n k}=0$. 
3C. The third Jacobi identity in the symplectic case. We already know from Section 2 that if $J_{1}=J_{2}=0$, then the third part $J_{3}$ of the super-Jacobi identity, for three 1-forms, vanishes. In view of the above, this is the case when the curvature and torsion both vanish. Here we give, as a check, a direct proof of this fact. We consider the super-Jacobi identity with three 1-forms, $\phi, \tau$ and $\eta$ :

$$
\begin{aligned}
{\left[\phi,[\tau, \eta]_{\bullet}\right]_{\bullet} } & =\left[\phi, \hbar \omega^{k q} \nabla_{k} \tau \wedge \nabla_{q} \eta+O\left(\hbar^{2}\right)\right]_{\bullet} \\
& =\hbar\left[\phi, \omega^{k q} \nabla_{k} \tau \wedge \nabla_{q} \eta\right]_{\bullet}+O\left(\hbar^{3}\right) \\
& =\hbar \omega^{k q}\left(\left[\phi, \nabla_{k} \tau\right]_{\bullet} \wedge \nabla_{q} \eta-\nabla_{k} \tau \wedge\left[\phi, \nabla_{q} \eta\right]_{\bullet}\right)+O\left(\hbar^{3}\right) \\
& =\hbar^{2} \omega^{k q} \omega^{n m}\left(\nabla_{n} \phi \wedge \nabla_{m} \nabla_{k} \tau \wedge \nabla_{q} \eta-\nabla_{k} \tau \wedge \nabla_{n} \phi \wedge \nabla_{m} \nabla_{q} \eta\right)+O\left(\hbar^{3}\right) \\
& =\hbar^{2} \omega^{k q} \omega^{n m}\left(\nabla_{n} \phi \wedge \nabla_{m} \nabla_{k} \tau \wedge \nabla_{q} \eta+\nabla_{k} \tau \wedge \nabla_{m} \nabla_{q} \eta \wedge \nabla_{n} \phi\right)+O\left(\hbar^{3}\right) .
\end{aligned}
$$

To show that the cyclic sum vanishes to $O\left(\hbar^{2}\right)$ we need the following 3-form to be zero:

$$
\begin{gathered}
\omega^{k q} \omega^{n m}\left(\nabla_{n} \phi \wedge \nabla_{m} \nabla_{k} \tau \wedge \nabla_{q} \eta+\nabla_{k} \tau \wedge \nabla_{m} \nabla_{q} \eta \wedge \nabla_{n} \phi+\nabla_{n} \tau \wedge \nabla_{m} \nabla_{k} \eta \wedge \nabla_{q} \phi\right. \\
\left.+\nabla_{k} \eta \wedge \nabla_{m} \nabla_{q} \phi \wedge \nabla_{n} \tau+\nabla_{n} \eta \wedge \nabla_{m} \nabla_{k} \phi \wedge \nabla_{q} \tau+\nabla_{k} \phi \wedge \nabla_{m} \nabla_{q} \tau \wedge \nabla_{n} \eta\right) \\
=\left(\omega^{k q} \omega^{n m}+\omega^{n k} \omega^{q m}\right) \nabla_{n} \phi \wedge \nabla_{m} \nabla_{k} \tau \wedge \nabla_{q} \eta \\
+\left(\omega^{k q} \omega^{n m}+\omega^{q n} \omega^{k m}\right) \nabla_{k} \tau \wedge \nabla_{m} \nabla_{q} \eta \wedge \nabla_{n} \phi \\
+\left(\omega^{k q} \omega^{n m}+\omega^{q n} \omega^{k m}\right) \nabla_{k} \eta \wedge \nabla_{m} \nabla_{q} \phi \wedge \nabla_{n} \tau
\end{gathered}
$$

3D. The generalised braiding associated to $\nabla$. If $A$ is an algebra equipped with a calculus and a left covariant derivative $\nabla: \Omega^{1} \rightarrow \Omega^{1} \otimes_{A} \Omega^{1}$ on it (meaning $\nabla(a \bullet \tau)=\mathrm{d} a \otimes_{A} \tau+a \bullet \nabla(\tau)$ for all $\left.a \in A, \tau \in \Omega^{1}\right)$ it may be possible to define a 'generalised braiding' [Madore 1999]:

$$
\bar{\sigma}: \Omega^{1} \otimes_{A} \Omega^{1} \rightarrow \Omega^{1} \otimes_{A} \Omega^{1}, \quad \sigma(\tau \otimes \mathrm{d} a)=\nabla(\tau \bullet a)-\nabla(\tau) \bullet a,
$$

where $\sigma$ is given on exact forms in the second argument and assumes that ker $\mathrm{d}$ is spanned by 1 . It is already a left module map and in well-behaved cases it extends also as a right module map to $\Omega^{1} \otimes \Omega^{1}$, in which case it follows using the Leibniz rule that it descends to an operator $\bar{\sigma}$ on $\Omega^{1} \otimes_{A} \Omega^{1}$.

In our case $\bullet$ is the deformed module structure on $\Omega^{1}(M)$, and we suppose a connection coinciding at lowest order with a given connection (such as the symplectic 
one above).

$$
\sigma(\tau \otimes \mathrm{d} x)=\mathrm{d} x \bar{\otimes} \tau-\nabla\left([x, \tau]_{\bullet}\right)+[x, \nabla \tau]_{\bullet}=\mathrm{d} x \bar{\otimes} \tau+\hbar \rho(\mathrm{d} x \otimes \tau)+O\left(\hbar^{2}\right),
$$

where we further suppose an expansion as shown. We write $\bar{\otimes}$ for the tensor product over $C^{\infty}(M)$.

Proposition 3.7. A left module map $\sigma$ defining a generalised braiding corresponds at the semiclassical level to $\rho$ obeying

$$
\eta \bar{\otimes} \gamma(x, \tau)+\rho(x \eta \otimes \tau)=x \rho(\eta \otimes \tau)=\rho(\eta \otimes \tau x)-\gamma(x, \eta) \bar{\otimes} \tau
$$

for all $x \in C^{\infty}(M)$ and $\tau, \eta \in \Omega^{1}(M)$. Moreover, when $\nabla$ is given by our symplectic connection in Section $3 A$, we have

$$
\rho(\eta \otimes \tau)=\omega^{j q} \omega^{i s} \eta_{j} \tau_{i} R_{s m k q} \mathrm{~d} x^{k} \bar{\otimes} \mathrm{d} x^{m}-\omega^{s q} \nabla_{s} \eta \bar{\otimes} \nabla_{q} \tau
$$

in a coordinate chart where $\nabla=\mathrm{d} x^{k} \bar{\otimes} \nabla_{k}$.

Proof. We suppose similarly that $\sigma(\eta \otimes \tau)=\tau \bar{\otimes} \eta+\hbar \rho(\tau \otimes \eta)+O(\hbar)$. That $\sigma$ is a left module map translates to

$$
\tau \bar{\otimes} a \cdot \eta+\hbar \rho(\tau \otimes a \bullet \eta)+O\left(\hbar^{2}\right)=a \bullet \tau \bar{\otimes} \eta+\hbar a \bullet \rho(\tau \otimes \eta)+O\left(\hbar^{2}\right) .
$$

Since $[a, \tau]_{\bullet}=\hbar \gamma(a, \tau)+O\left(\hbar^{2}\right)$ and $a$ passes through $\bar{\otimes}$, and since everything commutes at lowest order, we obtain the first equality. Similarly, that $\sigma$ is a right module map implies the second equality. That $\sigma$ descends to $\bar{\otimes}$ gives nothing new, appearing as equality of the first and last expressions.

Moreover, we compute

$$
\begin{aligned}
\rho\left(\mathrm{d} x^{j} \otimes \tau\right) & =-\mathrm{d} x^{k} \bar{\otimes} \nabla_{k} \gamma\left(x^{j}, \tau\right)+\gamma\left(x^{j}, \mathrm{~d} x^{k} \bar{\otimes} \nabla_{k}(\tau)\right) \\
& =-\mathrm{d} x^{k} \bar{\otimes}\left(\nabla_{k} \gamma\left(x^{j}, \tau\right)-\gamma\left(x^{j}, \nabla_{k}(\tau)\right)\right)+\gamma\left(x^{j}, \mathrm{~d} x^{k}\right) \bar{\otimes} \nabla_{k}(\tau) .
\end{aligned}
$$

Now set $X=\omega^{j q} \partial_{q}$, and then

$$
\rho\left(\mathrm{d} x^{j} \otimes \tau\right)=-\mathrm{d} x^{k} \bar{\otimes}\left(\nabla_{k} \nabla_{X}-\nabla_{X} \nabla_{k}\right)(\tau)+\nabla_{X}\left(\mathrm{~d} x^{k}\right) \bar{\otimes} \nabla_{k}(\tau) .
$$

In Darboux coordinates $\left[\partial_{k}, X\right]=0$, and

$$
\rho\left(\mathrm{d} x^{j} \otimes \tau\right)=-\mathrm{d} x^{k} \bar{\otimes} R\left(\partial_{k}, X\right)\left(\mathrm{d} x^{i}\right)+\nabla_{X}\left(\mathrm{~d} x^{k}\right) \bar{\otimes} \nabla_{k}(\tau) .
$$

Now we calculate the terms as

$$
\begin{aligned}
\mathrm{d} x^{k} \bar{\otimes} R\left(\partial_{k}, X\right)(\tau) & =\omega^{j q} \mathrm{~d} x^{k} \bar{\otimes} R\left(\partial_{k}, \partial_{q}\right)(\tau)=-\omega^{j q} \tau_{i} \mathrm{~d} x^{k} \bar{\otimes} R_{m k q}^{i} \mathrm{~d} x^{m}, \\
\nabla_{X}\left(\mathrm{~d} x^{k}\right) \bar{\otimes} \nabla_{k}(\tau) & =\omega^{j q} \nabla_{q}\left(\mathrm{~d} x^{k}\right) \bar{\otimes} \nabla_{k}(\tau) .
\end{aligned}
$$

Using these, from (3-6) we have

$$
\rho\left(\mathrm{d} x^{j} \otimes \tau\right)=\omega^{j q} \tau_{i} R_{m k q}^{i} \mathrm{~d} x^{k} \bar{\otimes} \mathrm{d} x^{m}-\omega^{s q} \nabla_{s}\left(\mathrm{~d} x^{j}\right) \bar{\otimes} \nabla_{q} \tau .
$$


Finally, we deduce the general form of $\rho$ from its properties above as $\rho\left(\eta_{j} \mathrm{~d} x^{j} \otimes \tau\right)=\omega^{j q} \tau_{i} \eta_{j} R_{m k q}^{i} \mathrm{~d} x^{k} \bar{\otimes} \mathrm{d} x^{m}-\omega^{s q} \eta_{j} \nabla_{s}\left(\mathrm{~d} x^{j}\right) \bar{\otimes} \nabla_{q} \tau-\mathrm{d} x^{j} \otimes \gamma\left(\eta_{j}, \tau\right)$.

Putting $\eta=\eta_{j} \mathrm{~d} x^{j}$ and assuming that $\nabla$ is the symplectic connection defined by $\gamma$ in Section 3A gives the result stated. We use the notations from [Gelfand et al. 1998].

It is also instructive to look at the braid relations, which justifies the term 'generalised braiding'. Writing $\bar{\sigma}_{12}$ for $\bar{\sigma}$ acting in the first and second tensor factors, and so on, the expression

$$
\left(\bar{\sigma}_{12} \bar{\sigma}_{23} \bar{\sigma}_{12}-\bar{\sigma}_{23} \bar{\sigma}_{12} \bar{\sigma}_{23}\right)(\xi \bar{\otimes} \eta \bar{\otimes} \tau)
$$

can be calculated to leading order in $\hbar$ as the quotient in $\left(\Omega^{1}\right)^{\bar{\otimes} 3}$ of

$$
\left(\tilde{\rho}_{12} \tilde{\rho}_{23}+\tilde{\rho}_{13} \tilde{\rho}_{23}+\tilde{\rho}_{12} \tilde{\rho}_{13}-\tilde{\rho}_{13} \tilde{\rho}_{12}-\tilde{\rho}_{23} \tilde{\rho}_{12}-\tilde{\rho}_{23} \tilde{\rho}_{13}\right)(\tau \otimes \eta \otimes \xi),
$$

where $\tilde{\rho}: \Omega^{1} \otimes \Omega^{1} \rightarrow \Omega^{1} \otimes \Omega^{1}$ is any lift of the output of $\rho$. The left-hand side is the 'classical Yang-Baxter expression'. In our case the natural lift determined by our coordinate system of the $\rho$ in Proposition 3.7 is

$$
\tilde{\rho}(\tau \otimes \eta)=\omega^{j q} \omega^{i s} \tau_{j} \eta_{i} R_{s m k q} \mathrm{~d} x^{k} \otimes \mathrm{d} x^{m}-\omega^{s q} \nabla_{s} \tau \otimes \nabla_{q} \eta .
$$

On substituting this into the classical Yang-Baxter expression, the part coming from the $\omega^{s q} \nabla_{s} \eta \otimes \nabla_{q} \xi$ term is

$$
\omega^{s q} \omega^{i j}\left(\nabla_{s} \otimes\left[\nabla_{q}, \nabla_{i}\right] \otimes \nabla_{j}+\nabla_{s} \otimes \nabla_{i} \otimes\left[\nabla_{q}, \nabla_{j}\right]+\left[\nabla_{s}, \nabla_{i}\right] \otimes \nabla_{q} \otimes \nabla_{j}\right)
$$

applied to $\zeta \otimes \eta \otimes \xi$. We deduce that if the curvature vanishes, then the classical Yang-Baxter equation holds for $\tilde{\rho}$ (even before we quotient), and hence $\bar{\sigma}$ satisfies the braid relation to $O\left(\hbar^{3}\right)$. We can also calculate

$$
\begin{aligned}
\left(\tilde{\rho}+\tilde{\rho}_{21}\right)(\tau \otimes \eta)= & \omega^{j q} \omega^{i s} R_{s m k q}\left(\tau_{j} \eta_{i} \mathrm{~d} x^{k} \otimes \mathrm{d} x^{m}+\eta_{j} \tau_{i} \mathrm{~d} x^{m} \otimes \mathrm{d} x^{k}\right) \\
& -\omega^{s q}\left(\nabla_{s} \tau \otimes \nabla_{q} \eta+\nabla_{q} \tau \otimes \nabla_{s} \eta\right) \\
= & \omega^{j q} \omega^{i s}\left(R_{s m k q}+R_{q k m s}\right) \tau_{j} \eta_{i} \mathrm{~d} x^{k} \otimes \mathrm{d} x^{m},
\end{aligned}
$$

so if the curvature vanishes, then $\tilde{\rho}$ is antisymmetric, and $\bar{\sigma}^{2}$ is the identity to $O\left(\hbar^{2}\right)$.

3E. Example: the noncommutative torus. Here we give an elementary example of the above, namely the noncommutative torus in an algebraic form generated by invertible $u, v$ with $v u=q u v$. The natural noncommutative calculus here is generated by

$$
\tau^{1}=u^{-1} \mathrm{~d} u, \quad \tau^{2}=v^{-1} \mathrm{~d} v
$$


with relations

$$
\left[x, \tau^{i}\right]=0, \quad \tau^{1} \wedge \tau^{2}+\tau^{2} \wedge \tau^{1}=0, \quad \tau^{i} \wedge \tau^{i}=0
$$

for all functions $x$ (this is not as trivial as it seems, it implies $v \mathrm{~d} u=q(\mathrm{~d} u) v$, etc.)

For our purposes we take $q=e^{-\hbar}$ as a formal expansion parameter, which we stress is only the 'trivial' part of the theory from the usual point of view (where one would have $\hbar=2 \pi \imath \theta$ with $\theta \in[0,1]$ and complete to a $C^{*}$-algebra). Next, in the classical limit we identify $u=e^{i \theta^{1}}$ and $v=e^{i \theta^{2}}$ in terms of local angle coordinates on $S^{1} \times S^{1}$. Along with their inverses they generate a subalgebra inside $C^{\infty}\left(S^{1} \times S^{2}\right)$ and induce a formal deformation of the whole algebra via a Moyal-type product. For our purposes all structures can be extracted just from the subalgebra with the above generators. Note that $\tau^{i}=\imath \mathrm{d} \theta^{i}$.

We start with the Poisson structure which is clearly

$$
\{u, v\}=1=-\{v, u\}, \quad\{u, u\}=\{v, v\}=0
$$

which means $\left(\omega^{i j}\right)=\left(\begin{array}{rr}0 & 1 \\ -1 & 0\end{array}\right)$ in the $\tau^{i}$ basis. To see this, note that

$$
\mathrm{d} x=\left(\partial_{i} x\right) \tau^{i} ; \quad \partial_{1}=u \frac{\partial}{\partial u}, \quad \partial_{2}=v \frac{\partial}{\partial v} .
$$

Then $\{u, y\}=\partial_{2} y$ and $\{v, y\}=-\partial_{1} y$ for all $y$ since $\hat{u}$ and $\hat{v}$ extend as derivations. Then since each $\hat{y}$ is a derivation, we similarly deduce

$$
\{x, y\}=\omega^{i j}\left(\partial_{i} x\right)\left(\partial_{j} y\right)
$$

with $\omega^{i j}$ as above. The symplectic form is $\tau^{1} \wedge \tau^{2}$ up to normalisation.

Finally, since $\tau^{i}$ are central, we know that $\gamma\left(x, \tau^{i}\right)=0$ for $i=1,2$ and all functions $x$. Hence

$$
\nabla_{\hat{x}}\left(\eta_{i} \tau^{i}\right)=\gamma\left(x, \eta_{i} \tau^{i}\right)=\left\{x, \eta_{i}\right\} \tau^{i}
$$

by the connection property. This is our symplectic connection. Compatibility in the sense of (2-5) holds as it must, reducing to

$$
\partial_{i}\{x, y\}=\left\{\partial_{i} x, y\right\}+\left\{x, \partial_{i} y\right\}
$$

which holds because $\omega^{i j}$ are constant. We may also compute its curvature:

$$
\begin{aligned}
R(\hat{x}, \hat{y}) a_{i} \tau^{i} & =\nabla_{\hat{x}}\left\{y, a_{i}\right\} \tau^{i}-\nabla_{\hat{y}}\left\{x, a_{i}\right\} \tau^{i}-\nabla_{\widehat{\{x, y\}}} a_{i} \tau^{i} \\
& =\left\{x,\left\{y, a_{i}\right\}\right\} \tau^{i}-\left\{y,\left\{x, a_{i}\right\}\right\} \tau^{i}-\left\{\{x, y\}, a_{i}\right\} \tau^{i}=0
\end{aligned}
$$

as it must since the above quantum differential calculus is associative to all orders. 
Moreover,

$$
\begin{aligned}
\langle T(\hat{x}, \hat{y}), \mathrm{d} u\rangle & =\left\langle\hat{x}, \nabla_{\hat{y}} \mathrm{~d} u\right\rangle-\left\langle\hat{y}, \nabla_{\hat{x}} \mathrm{~d} u\right\rangle \\
& =\left\langle\hat{x},\left\{y, \partial_{i} u\right\} \tau^{i}\right\rangle-\left\langle\hat{y},\left\{x, \partial_{i} u\right\} \tau^{i}\right\rangle \\
& =\{y, u\}\left\langle\hat{x}, \tau^{1}\right\rangle-\{x, u\}\left\langle\hat{y}, \tau^{1}\right\rangle \\
& =\{y, u\} u^{-1}\{x, u\}-\{x, u\} u^{-1}\{y, u\}=0,
\end{aligned}
$$

since $\left\langle\hat{x}, u^{-1} \mathrm{~d} u\right\rangle=u^{-1} \hat{x}(u)=u^{-1}\{x, u\}$. Similarly $\langle T(\hat{x}, \hat{y}), \mathrm{d} v\rangle=0$, and hence $T(\hat{x}, \hat{y})=0$. Hence the torsion vanishes as it must by Proposition 3.3. So we have a flat torsion free (and symplectic) connection on the standard torus induced by noncommutative geometry.

Finally, the semiclassical braiding in Proposition 3.7 for this connection, using the fact that the curvature is zero, and (3-8), comes out to be $\rho\left(\tau^{i} \otimes \tau^{j}\right)=0$ so that

$$
\rho\left(\eta_{i} \tau^{i} \otimes \xi_{j} \tau^{j}\right)=-\left\{\eta_{i}, \xi_{j}\right\} \tau^{i} \bar{\otimes} \tau^{j}
$$

on general 1-forms with coefficients $\eta_{i}, \xi_{j}$ in the left-invariant basis. Note also that $\bar{\sigma}\left(\tau_{i} \bar{\otimes} \tau_{j}\right)=\tau_{j} \bar{\otimes} \tau_{i}+O\left(\hbar^{2}\right)$ agrees with the quantum case [Beggs 2003] where it was found that the unique braiding on the noncommutative torus which is compatible with interior products for the given differential structure is the usual flip on the $\tau^{i}$.

\section{Application to Poisson-Lie groups}

In this section we are going to analyse the meaning of the deformation results of Section 2 in a different geometrical setting, namely that of Poisson-Lie groups. This is at the other extreme from the previous section in that these are never symplectic (the Poisson bracket being degenerate at the group identity). On the other hand one can impose the group symmetry and reduce expressions to the Lie algebra level which has its own interest.

Poisson-Lie groups are the semiclassical data for Hopf algebras or quantum groups. At the Lie level they correspond to a Lie bialgebra $(\mathfrak{g}, \delta)$ where $\delta: \mathfrak{g} \rightarrow \mathfrak{g} \otimes \mathfrak{g}$ is a Lie cobracket making $\mathfrak{g}^{*}$ a Lie algebra and forming a cocycle in $Z_{\text {ad }}^{1}(\mathfrak{g}, \mathfrak{g} \otimes \mathfrak{g})$. This is like an infinitesimal quantum group. Exponentiating the Lie algebra to a group $G$ and $\delta$ to $D \in Z_{\text {Ad }}^{1}(G, \mathfrak{g} \otimes \mathfrak{g})$ and extending the latter to an invariant bivector defines a Poisson bracket. Working backwards one has axioms of a Poisson-Lie group and its tangent space at the identity is a Lie bialgebra. The notions are due to Drinfeld. An introduction can be found in [Majid 1995].

Now, for a Hopf algebra $H$ we have the following notions of covariance of a differential structure, which we shall aim to semiclassicalise. Note that a Hopf algebra acts and coacts on itself from both sides via the product and coproduct 
respectively. Here the coproduct is a map $\Delta: H \rightarrow H \otimes H$. We will often use the Sweedler notation $\Delta a=a_{(1)} \otimes a_{(2)}$, (id $\left.\otimes \Delta\right) \Delta a=a_{(1)} \otimes a_{(2)} \otimes a_{(3)}$, etc., for it.

Definition 4.1. A differential calculus $\left(\Omega^{1}, \mathrm{~d}\right)$ on a Hopf algebra $H$ is called left covariant if

(1) $\Omega^{1}$ is a left comodule for $H$ with coaction $\Delta_{L}: \Omega^{1} \rightarrow H \otimes \Omega^{1}$.

(2) $\Delta_{L}: \Omega^{1} \rightarrow H \otimes \Omega^{1}$ is a bimodule map (with the tensor bimodule structure on $\left.H \otimes \Omega^{1}\right)$.

(3) The map d:H $\rightarrow \Omega^{1}$ is a comodule map.

Similarly for a right-covariant calculus with structure map $\Delta_{R}: \Omega^{1} \rightarrow \Omega^{1} \otimes H$.

A calculus is called bicovariant if it is both left and right covariant and

(4) The left and right coactions on $\Omega^{1}$ commute (a bicomodule).

Condition (3) fully determines the left coaction (similarly the right) if they exist, since every element of $\Omega^{1}$ is a linear combination of ones of the form $a \mathrm{~d} b$, so the coations are induced canonically from the 'group translation' or coproduct in the invariant case.

4A. Semiclassical left covariance condition. The functions on a Poisson Lie group $G$ typically deform to a noncommutative Hopf algebra $H$, with the commutator of two functions being given by the Poisson bracket to first order in $\hbar$. We suppose that the differential calculus on $H$ is a deformation of the standard (commutative) differential calculus in the sense that we have discussed earlier. We now ask what left, right (and later bi) covariance of the differential calculus means in terms of the connection $\gamma$. To this end we use the following lemma for left (similarly right) covariant calculi on a Hopf algebra $H$ :

Lemma 4.2. Write $\Delta_{L}(\tau)=\tau_{(1)} \otimes \tau_{(\infty)}$ and $\Delta_{R}(\tau)=\tau_{(0)} \otimes \tau_{(1)}$. Then for $a \in H$ and $\tau \in \Omega^{1}$,

$$
\begin{aligned}
& \left((\mathrm{id} \otimes[,]) \circ \Delta_{L}-\Delta_{L} \circ[,]\right)(a \otimes \tau)=-\left[a_{(1)}, \tau_{(1)}\right] \otimes \tau_{(\infty)} a_{(2)} \in H \otimes \Omega^{1}, \\
& \left(([,] \otimes \mathrm{id}) \circ \Delta_{R}-\Delta_{R} \circ[,]\right)(a \otimes \tau)=\tau_{(0)} a_{(1)} \otimes\left[\tau_{(1)}, a_{(2)}\right] \in \Omega^{1} \otimes H .
\end{aligned}
$$

Proof. These results follow from the equations

$$
\Delta_{L}[a, \tau]=\Delta_{L}(a \tau-\tau a)=a_{(1)} \tau_{(1)} \otimes a_{(2)} \tau_{(\infty)}-\tau_{(1)} a_{(1)} \otimes \tau_{(\infty)} a_{(2)},
$$

$(\mathrm{id} \otimes[],) \Delta_{L}(a \otimes \tau)=(\mathrm{id} \otimes[]),\left(a_{(1)} \tau_{(1)} \otimes a_{(2)} \otimes \tau_{(\infty)}\right)$

$$
\begin{aligned}
& =a_{(1)} \tau_{(1)} \otimes\left(a_{(2)} \tau_{(\infty)}-\tau_{(\infty)} a_{(2)}\right), \\
\Delta_{R}[a, \tau] & =\Delta_{R}(a \tau-\tau a)=a_{(1)} \tau_{(0)} \otimes a_{(2)} \tau_{(1)}-\tau_{(0)} a_{(1)} \otimes \tau_{(1)} a_{(2)},
\end{aligned}
$$

$([,] \otimes \mathrm{id}) \Delta_{R}(a \otimes \tau)=([,] \otimes \mathrm{id})\left(a_{(1)} \otimes \tau_{(0)} \otimes a_{(2)} \tau_{(1)}\right)$

$$
=\left(a_{(1)} \tau_{(0)}-\tau_{(0)} a_{(1)}\right) \otimes a_{(2)} \tau_{(1)} .
$$


For a Lie group $G$, there is a left $G$-action on functions on $G$ given by

$$
(g \triangleright a)(h)=a\left(g^{-1} h\right) .
$$

The left multiplication map $L_{g}: G \rightarrow G$ has a derivative $L_{g *}: T_{h} G \rightarrow T_{g h} G$ at all $h \in G$, and the dual of this map is $L_{g}^{*}: T_{g h}^{*} G \rightarrow T_{h}^{*} G$. There is a left action on $\tau \in T^{*} G$ given by $(g \triangleright \tau)(h)=L_{g^{-1}}^{*}\left(\tau\left(g^{-1} h\right)\right)$ for all $h$. In terms of the algebra of functions on $G$, we have (in an appropriate setting) a left coaction $\left(\Delta_{L} a\right)(g, h)=a(g h)=\left(g^{-1} \triangleright a\right)(h)$, and a left coaction on the 1-forms $\left(\Delta_{L} \tau\right)(g, h)=L_{g}^{*}(\tau(g h))=\left(g^{-1} \triangleright \tau\right)(h)$. In our case we work with $C^{\infty}(G)$ rather than algebraically, hence we work directly with actions and group multiplication rather than such coactions and coproduct. When working abstractly on the group (or any other) manifold we employ the notations

$$
v(a)(g)=D_{(g ; v(g))} a=a^{\prime}(g ; v(g))=\left.\frac{\mathrm{d}}{\mathrm{d} t}\right|_{0} a(g(t))
$$

for the action of a vector field $v$, where $g(t)$ is a curve with $g(0)=g$ and tangent $v(g)$ there. Also, if $v \in \mathfrak{g}=T_{e} G$ we write the associated left invariant vector field $L_{* v}$ with values $L_{g *} v \in T_{g} G$, or simply $g v$ in a shorthand. We similarly have right multiplication $R_{g}: G \rightarrow G$, etc., and right invariant vector field $R_{* v}$ generated by $v \in \mathfrak{g}$.

To interpret the left invariance formula in Lemma 4.2 for a Poisson Lie group $G$, we remember that, up to $O(\hbar)$, we have the same coaction formulae as in the Lie group case. We also need to trivialise the cotangent bundle by a map $T^{*} G \rightarrow G \times \mathfrak{g}^{*}$ defined by $(g ; \xi) \mapsto\left(g, L_{g}^{*} \xi\right)$ for $\xi \in T_{g}^{*} G$. Given a section $\tau$ of $T^{*} G$ we define a section $\tilde{\tau}$ of the trivial $\mathfrak{g}^{*}$ bundle (a function on $G$ with values in $\mathfrak{g}^{*}$ ) by

$$
\tilde{\tau}(g)=L_{g}^{*}(\tau(g))=\left(g^{-1} \triangleright \tau\right)(e)
$$

where $e$ is the group identity.

Lemma 4.3. The semiclassicalisation $\gamma$ of a left-invariant calculus on $G$ obeys

$$
\tilde{\gamma}(a, \tilde{\tau})(g h)-\tilde{\gamma}\left(g^{-1} \triangleright a, \widehat{g^{-1} \triangleright \tau}\right)(h)=a^{\prime}\left(g h ; \omega^{[1]}(g) h\right) \tilde{\tau}^{\prime}\left(g h ; \omega^{[2]}(g) h\right)
$$

as the lowest order part of the condition in Lemma 4.2.

Proof. To calculate $\left[a_{(1)}, \tau_{(1)}\right] \otimes \tau_{(\infty)} a_{(2)}$ we use the Poisson bracket with bivector $\omega=\omega^{[1]} \otimes \omega^{[2]}$ whereby $\{a, b\}=D_{\left(g ; \omega^{[1]}(g)\right)}(a) D_{\left(g ; \omega^{[2]}(g)\right)}(b)$ for functions $a, b$. Then

$$
\begin{aligned}
\left(\left[a_{(1)}, \tau_{(1)}\right] \otimes \tau_{(\infty)} a_{(2)}\right)(g, h) & =\hbar\left(D_{\left(g ; \omega^{[1]}(g)\right)} a(g h)\right)\left(D_{\left(g ; \omega^{[2]}(g)\right)} L_{g}^{*}(\tau(g h))\right)+O\left(\hbar^{2}\right) \\
\Delta_{L}[a, \tau](g, h) & =\hbar L_{g}^{*}(\gamma(a, \tau)(g h))+O\left(\hbar^{2}\right),
\end{aligned}
$$

$(\mathrm{id} \otimes[],) \Delta_{L}(a \otimes \tau)(g, h)=\hbar \gamma\left(g^{-1} \triangleright a, g^{-1} \triangleright \tau\right)(h)+O\left(\hbar^{2}\right)$. 
Now we can evaluate the first half of Lemma 4.2 at $(g, h)$ to order $\hbar$ and apply $L_{h}^{*}$ to both sides to get

$$
\begin{aligned}
L_{g h}^{*}(\gamma(a, \tau)(g h))-L_{h}^{*}\left(\gamma \left(g^{-1} \triangleright a,\right.\right. & \left.\left.g^{-1} \triangleright \tau\right)(h)\right) \\
& =\left(D_{\left(g ; \omega^{[1]}(g)\right)} a(g h)\right)\left(D_{\left(g ; \omega^{[2]}(g)\right)} L_{g h}^{*}(\tau(g h))\right) .
\end{aligned}
$$

This is

$$
\begin{aligned}
\left((g h)^{-1} \triangleright \gamma(a, \tau)\right)(e)-\left(h^{-1} \triangleright\right. & \left.\gamma\left(g^{-1} \triangleright a, g^{-1} \triangleright \tau\right)\right)(e) \\
& \left.=\left(D_{\left(g ; \omega^{[1]}(g)\right)} a(g h)\right)\left(D_{\left(g ; \omega^{[2]}(g)\right)}\left((g h)^{-1} \triangleright \tau\right)(e)\right)\right),
\end{aligned}
$$

which is the equation stated. Note that by definition $\tilde{\gamma}(a, \tilde{\tau})=\widetilde{\gamma(a, \tau)}$, so that everything in the ${ }^{\sim}$ notation is referred to functions on $G$.

Conversely, if we have only a preconnection as semiclassical datum for a calculus, we say that it is left-invariant if the condition in the lemma holds. We now give a different characterisation.

Definition 4.4. On the trivial bundle $G \times \mathfrak{g}^{*} \rightarrow G$ we define the bilinear-valued function $\Xi: G \times \mathfrak{g}^{*} \times \mathfrak{g}^{*} \rightarrow \mathfrak{g}^{*}$ by

$$
\begin{aligned}
\tilde{\gamma}(a, s)(g) & =a^{\prime}\left(g ; \omega^{[1]}(g)\right) s^{\prime}\left(g ; \omega^{[2]}(g)\right)+\Xi\left(g ; \hat{L}_{a}(g), s(g)\right) \\
& =\{a, s\}(g)+\Xi\left(g, \hat{L}_{a}(g), s(g)\right),
\end{aligned}
$$

where $a \in C^{\infty}(G), s \in C^{\infty}\left(G, \mathfrak{g}^{*}\right)$ and $\hat{L}_{a}: G \rightarrow \mathfrak{g}^{*}$ is the left invariant derivative $\hat{L}_{a}(g)(v)=a^{\prime}(g ; g v)$.

In the second expression we have extended the notation for the Poisson bracket to include $\mathfrak{g}^{*}$-valued functions, and also note that $\hat{L}_{a}=\widetilde{\mathrm{d} a}$.

Proposition 4.5. A preconnection $\gamma$ is left invariant if and only if $\Xi: G \times \mathfrak{g}^{*} \times \mathfrak{g}^{*} \rightarrow$ $\mathfrak{g}^{*}$ is independent of the $G$ variable.

Proof. To apply the condition in Lemma 4.3, we need to note that $\left.\widetilde{\left(g^{-1} \triangleright \tau\right.}\right)(h)=$ $\tilde{\tau}(g h)$, and then

$$
\begin{aligned}
& a^{\prime}\left(g h ; \omega^{[1]}(g h)\right) \tilde{\tau}^{\prime}\left(g h ; \omega^{[2]}(g h)\right)+\Xi\left(g h, \hat{L}_{a}(g h), \tilde{\tau}(g h)\right) \\
& -a^{\prime}\left(g h ; g \omega^{[1]}(h)\right) \tilde{\tau}^{\prime}\left(g h ; g \omega^{[2]}(h)\right)-\Xi\left(h, \hat{L}_{g^{-1} \triangleright a}(h), \tilde{\tau}(g h)\right) \\
& \quad=a^{\prime}\left(g h ; \omega^{[1]}(g) h\right) \tilde{\tau}^{\prime}\left(g h ; \omega^{[2]}(g) h\right) .
\end{aligned}
$$

In $T G \otimes T G$ we have $\omega(g h)-g \omega(h)-\omega(g) h=0$, so the last equation reduces to

$$
\Xi\left(g h, \hat{L}_{a}(g h), \tilde{\tau}(g h)\right)=\Xi\left(h, \hat{L}_{g^{-1} \triangleright a}(h), \tilde{\tau}(g h)\right) .
$$

A bit of calculation shows that $\hat{L}_{g^{-1} \triangleright a}(h)=\hat{L}_{a}(g h)$, so we are left with

$$
\Xi(g h, \phi, \psi)=\Xi(h, \phi, \psi) \text { for all } \phi, \psi \in \mathfrak{g}^{*} \text { and all } g, h \in G .
$$


We deduce that $\Xi(h, \phi, \psi)$ is independent of $h \in G$.

Proposition 4.6. A left invariant preconnection $\tilde{\gamma}$ corresponding to $\Xi: \mathfrak{g}^{*} \otimes \mathfrak{g}^{*} \rightarrow \mathfrak{g}^{*}$ is compatible in the sense of (2-5) if and only if

$$
\Xi(\phi, \psi)-\Xi(\psi, \phi)=[\phi, \psi]_{\mathfrak{g}^{*}}
$$

Proof. The left invariant trivialisation of $T^{*} G$ gives the following form of (2-5):

$$
\tilde{\gamma}\left(x, \hat{L}_{y}\right)-\tilde{\gamma}\left(y, \hat{L}_{x}\right)=\hat{L}_{\{x, y\}} .
$$

This can be rearranged to give

$$
\Xi\left(\hat{L}_{x}, \hat{L}_{y}\right)-\Xi\left(\hat{L}_{y}, \hat{L}_{x}\right)=\hat{L}_{\{x, y\}}-\left\{x, \hat{L}_{y}\right\}+\left\{y, \hat{L}_{x}\right\} .
$$

We only have to evaluate this equation at the identity $e \in G$. All Poisson brackets evaluated at the identity vanish as $\omega(e)=0$, so to find the right-hand side of (4-2) we only need

$$
\begin{aligned}
& \hat{L}_{\{x, y\}}(g)(v)=D_{(g ; g v)}\{x, y\}, \\
& \hat{L}_{\{x, y\}}(e)(v)=x^{\prime}\left(e ; \omega^{[1]^{\prime}}(e ; v)\right) y^{\prime}\left(e ; \omega^{[2]}(e)\right)+x^{\prime}\left(e ; \omega^{[1]}(e)\right) y^{\prime}\left(e ; \omega^{[2]^{\prime}}(e ; v)\right) .
\end{aligned}
$$

Setting $\phi(v)=x^{\prime}(e ; v)$ and $\psi(v)=y^{\prime}(e ; v)$, we see that evaluating (4-2) at $e$ gives

$$
\Xi(\phi, \psi)(v)-\Xi(\psi, \phi)(v)=(\phi \otimes \psi)\left(\omega^{\prime}(e ; v)\right)=(\phi \otimes \psi)(\delta(v))=[\phi, \psi]_{\mathfrak{g}^{*}}(v) .
$$

4B. Left covariance in the quasitriangular case. The quasitriangular case is the most important one, because the standard $q$-deformation quantum groups quantise Poisson-Lie groups of this type. In this case there are some useful simplifications which will be needed in the next section. We recall that a Lie bialgebra $\mathfrak{g}$ is called quasitriangular if the Lie cobracket $\delta$ is of the form $\delta v=\operatorname{ad}_{v}(r)$ for all $v \in \mathfrak{g}$, where $r=r^{[1]} \otimes r^{[2]} \in \mathfrak{g} \otimes \mathfrak{g}$ obeys the Classical Yang-Baxter equations

$$
\llbracket r, r \rrbracket \equiv\left[r_{12}, r_{13}\right]+\left[r_{12}, r_{23}\right]+\left[r_{13}, r_{23}\right]=0 .
$$

The expression here is the Schouten bracket and the numbers refer to the position in $\mathfrak{g} \otimes \mathfrak{g} \otimes \mathfrak{g}$. In this case $\omega(g)=g r-r g$ for all $g \in G$. Set $r_{ \pm}=\left(r \pm r_{21}\right) / 2$, where $r_{21}=r^{[2]} \otimes r^{[1]}$. Then $r_{+}$is necessarily ad-invariant, as is the element

$$
n \equiv \llbracket r_{-}, r_{-} \rrbracket=-\llbracket r_{+}, r_{+} \rrbracket=\left[r_{+12}, r_{+23}\right] .
$$

A quasitriangular Lie bialgebra is called triangular if $r_{+}=0$.

Corollary 4.7. If $\mathfrak{g}$ is quasitriangular, a left invariant preconnection $\tilde{\gamma}$ is compatible if and only if the map $\hat{\Xi}: \mathfrak{g}^{*} \otimes \mathfrak{g}^{*} \rightarrow \mathfrak{g}^{*}$ defined by

$$
\hat{\mathrm{\Xi}}(\phi, \psi)=\Xi(\phi, \psi)-\phi\left(r_{-}^{[1]}\right) \mathrm{ad}_{r_{-}[2]}^{*} \psi
$$

is symmetric. Here $\operatorname{ad}_{v}^{*} \psi(w)=\psi([w, v])$. 
Proof. Because $r_{+}$is ad-invariant, the result of Proposition 4.6 can be written as $\Xi(\phi, \psi)(v)-\Xi(\psi, \phi)(v)=(\phi \otimes \psi)\left(\left[v, r_{-}{ }^{[1]}\right] \otimes r_{-}{ }^{[2]}+r_{-}{ }^{[1]} \otimes\left[v, r_{-}{ }^{[2]}\right]\right)$.

Then we have the following, which is zero by the antisymmetry of $r_{-}$: $\hat{\Xi}(\phi, \psi)(v)-\hat{\Xi}(\psi, \phi)(v)=\phi\left(\left[v, r_{-}{ }^{[1]}\right]\right) \psi\left(r_{-}{ }^{[2]}\right)+\phi\left(r_{-}{ }^{[1]}\right) \psi\left(\left[v, r_{-}{ }^{[2]}\right]\right)$

$$
-\phi\left(r_{-}{ }^{[1]}\right) \psi\left(\left[v, r_{-}{ }^{[2]}\right]\right)+\psi\left(r_{-}{ }^{[1]}\right) \phi\left(\left[v, r_{-}{ }^{[2]}\right]\right) .
$$

Proposition 4.8. In the quasitriangular case, the first super-Jacobi identity is equivalent to the following equation for all $\phi, \psi, \zeta \in \mathfrak{g}^{*}$ :

$\Xi(\phi, \Xi(\psi, \zeta))-\Xi(\psi, \Xi(\phi, \zeta))=\phi\left(r_{-}^{[1]}\right) \Xi\left(\operatorname{ad}_{r_{-}[2]}^{*} \psi, \zeta\right)+\psi\left(r_{-}^{[2]}\right) \Xi\left(\operatorname{ad}_{r_{-}[1]}^{*} \phi, \zeta\right)$.

Proof. The first super-Jacobi identity reduces to showing that

$$
\tilde{\gamma}(x, \tilde{\gamma}(y, s))-\tilde{\gamma}(y, \tilde{\gamma}(x, s))=\tilde{\gamma}(\{x, y\}, s) .
$$

If we use the Poisson bracket notation, we can write

$$
\begin{aligned}
\tilde{\gamma}(x, \tilde{\gamma}(y, s))(g)=\{x,\{y, s\}\} & (g)+\left\{x, \Xi\left(\hat{L}_{y}(g), s(g)\right)\right\} \\
& +\Xi\left(\hat{L}_{x}(g),\{y, s\}(g)\right)+\Xi\left(\hat{L}_{x}(g), \Xi\left(\hat{L}_{y}(g), s(g)\right)\right) .
\end{aligned}
$$

Then the Jacobi identity for the Poisson bracket shows that all the double Poisson bracket terms in (4-3) cancel, so we get

$$
\begin{aligned}
\Xi\left(\hat{L}_{\{x, y\}}(g), s(g)\right) \\
=\left\{x, \Xi\left(\hat{L}_{y}(g), s(g)\right)\right\}+\Xi\left(\hat{L}_{x}(g),\{y, s\}(g)\right)+\Xi\left(\hat{L}_{x}(g), \Xi\left(\hat{L}_{y}(g), s(g)\right)\right) \\
\quad-\left\{y, \Xi\left(\hat{L}_{x}(g), s(g)\right)\right\}-\Xi\left(\hat{L}_{y}(g),\{x, s\}(g)\right)-\Xi\left(\hat{L}_{y}(g), \Xi\left(\hat{L}_{x}(g), s(g)\right)\right) .
\end{aligned}
$$

Using the equation

$$
\left\{x, \Xi\left(\hat{L}_{y}(g), s(g)\right)\right\}=\Xi\left(\left\{x, \hat{L}_{y}\right\}(g), s(g)\right)+\Xi\left(\hat{L}_{y}(g),\{x, s\}(g)\right),
$$

we find

$$
\begin{array}{r}
\Xi\left(\hat{L}_{\{x, y\}}(g), s(g)\right)=\Xi\left(\left\{x, \hat{L}_{y}\right\}(g), s(g)\right)+\Xi\left(\hat{L}_{x}(g), \Xi\left(\hat{L}_{y}(g), s(g)\right)\right) \\
-\Xi\left(\left\{y, \hat{L}_{x}\right\}(g), s(g)\right)-\Xi\left(\hat{L}_{y}(g), \Xi\left(\hat{L}_{x}(g), s(g)\right)\right) .
\end{array}
$$

Now calculate

$$
\begin{aligned}
\hat{L}_{\{x, y\}}(g)(v) & =D_{(g ; g v)}\left(x^{\prime}\left(g ; \omega^{[1]}(g)\right) y^{\prime}\left(g ; \omega^{[2]}(g)\right)\right), \\
\left\{x, \hat{L}_{y}\right\}(g)(v) & =x^{\prime}\left(g ; \omega^{[1]}(g)\right) D_{\left(g ; \omega^{[2]}(g)\right)} y^{\prime}(g ; g v),
\end{aligned}
$$

so using the antisymmetry of $\omega$ and the Lie bracket of vector fields,

$$
\begin{aligned}
\hat{L}_{\{x, y\}}(g)(v) & -\left\{x, \hat{L}_{y}\right\}(g)(v)+\left\{y, \hat{L}_{x}\right\}(g)(v) \\
& =x^{\prime}\left(g ; \omega^{[1]}(g)\right) y^{\prime}\left(g ;\left[g v, \omega^{[2]}(g)\right]\right)+x^{\prime}\left(g ;\left[g v, \omega^{[1]}(g)\right]\right) y^{\prime}\left(g ; \omega^{[2]}(g)\right) .
\end{aligned}
$$


Using the Ad-invariance of $r_{+}$we write $\omega=g r_{-}-r_{-} g$, which gives the answer.

We end with some lemmas to be used later.

Lemma 4.9. In terms of $\hat{\boldsymbol{E}}$, the first super-Jacobi identity is equivalent to the vanishing of the following expression for all $\phi, \psi, \zeta \in \mathfrak{g}^{*}$, where $n=\llbracket r_{-}, r_{-} \rrbracket$ :

$$
\begin{aligned}
\hat{\Xi}(\phi, \hat{\Xi}(\psi, \zeta))-\hat{\Xi} & (\psi, \hat{\Xi}(\phi, \zeta))+\phi\left(n^{[1]}\right) \psi\left(n^{[2]}\right) \operatorname{ad}_{n^{[3]}}^{*} \zeta \\
+\phi\left(r_{-}{ }^{[1]}\right) & \left(\operatorname{ad}_{r_{-}{ }^{[2]}}^{*} \hat{\Xi}(\psi, \zeta)-\hat{\Xi}\left(\psi, \operatorname{ad}_{r_{-}[2]}^{*} \zeta\right)-\hat{\Xi}\left(\operatorname{ad}_{r_{-}[2]}^{*} \psi, \zeta\right)\right) \\
& -\psi\left(r_{-}{ }^{[1]}\right)\left(\operatorname{ad}_{r_{-}[2]}^{*} \hat{\Xi}(\phi, \zeta)-\hat{\Xi}\left(\phi, \operatorname{ad}_{r_{-}[2]}^{*} \zeta\right)-\hat{\Xi}\left(\operatorname{ad}_{r_{-}[2]}^{*} \phi, \zeta\right)\right) .
\end{aligned}
$$

Proof. Let $t$ be an independent copy of $r$. By the definition of $\hat{\Xi}$,

$$
\begin{aligned}
\Xi(\phi, \Xi(\psi, \zeta))= & \hat{\Xi}(\phi, \hat{\Xi}(\psi, \zeta))+\psi\left(r_{-}{ }^{[1]}\right) \hat{\Xi}\left(\phi, \operatorname{ad}_{r_{-}{ }^{[2]}}^{*} \zeta\right) \\
& +\phi\left(r_{-}{ }^{[1]}\right) \operatorname{ad}_{r_{-}\left[{ }^{[2]}\right.}^{*} \hat{\Xi}(\psi, \zeta)+\phi\left(r_{-}{ }^{[1]}\right) \psi\left(t_{-}{ }^{[1]}\right) \operatorname{ad}_{r_{-}[2]}^{*} \operatorname{ad}_{t_{-}[2]}^{*} \zeta,
\end{aligned}
$$

$\phi\left(r_{-}{ }^{[1]}\right) \Xi\left(\operatorname{ad}_{r_{-}[2]}^{*} \psi, \zeta\right)=\phi\left(r_{-}{ }^{[1]}\right) \hat{\Xi}\left(\operatorname{ad}_{r_{-}[2]}^{*} \psi, \zeta\right)+\phi\left(r_{-}{ }^{[1]}\right)\left(\operatorname{ad}_{r_{-}[2]}^{*} \psi\right)\left(t_{-}^{[1]}\right) \operatorname{ad}_{t_{-}\left[{ }^{[2]}\right.}^{*} \zeta$.

By Proposition 4.8, we get the vanishing of

$$
\begin{aligned}
& \hat{\Xi}(\phi, \hat{\Xi}(\psi, \zeta))-\hat{\Xi}(\psi, \hat{\Xi}(\phi, \zeta))+\psi\left(r_{-}{ }^{[1]}\right) \hat{\Xi}\left(\phi, \operatorname{ad}_{r_{-}{ }^{[2]}}^{*} \zeta\right) \\
& \quad-\phi\left(r_{-}{ }^{[1]}\right) \hat{\Xi}\left(\psi, \operatorname{ad}_{r_{-}{ }^{[2]}}^{*} \zeta\right)+\phi\left(r_{-}{ }^{[1]}\right) \operatorname{ad}_{r_{-}[2]}^{*} \hat{\Xi}(\psi, \zeta)-\psi\left(r_{-}{ }^{[1]}\right) \operatorname{ad}_{r_{-}{ }^{[2]}} \hat{\Xi}(\phi, \zeta) \\
& \quad-\phi\left(r_{-}{ }^{[1]}\right) \hat{\Xi}\left(\operatorname{ad}_{r_{-}[2]}^{*} \psi, \zeta\right)+\psi\left(r_{-}{ }^{[1]}\right) \hat{\Xi}\left(\operatorname{ad}_{r_{-}[2]}^{*} \phi, \zeta\right)+\phi\left(n^{[1]}\right) \psi\left(n^{[2]}\right) \operatorname{ad}_{n^{[3]}}^{*} \zeta,
\end{aligned}
$$

and this can be rearranged to give the answer.

Lemma 4.10. Given $\hat{\boldsymbol{\Xi}}$, we recover $\gamma$ by

$$
\gamma(x, \mathrm{~d} y)(g)=x^{\prime}\left(g ; \omega^{[1]}(g)\right) \mathrm{d} y^{\prime}\left(g ; \omega^{[2]}(g)\right)+L_{g^{-1}}^{*} \hat{\Xi}\left(\hat{L}_{x}(g), \hat{L}_{y}(g)\right) .
$$

Proof. Evaluating Definition 4.4 against $v \in \mathfrak{g}$ we have

$$
\begin{aligned}
\tilde{\gamma}\left(x, \hat{L}_{y}\right)(g)(v) & =\left\{x, \hat{L}_{y}\right\}(g)(v)+\Xi\left(\hat{L}_{x}(g), \hat{L}_{y}(g)\right)(v) \\
= & x^{\prime}\left(g ; \omega^{[1]}(g)\right) D_{(g ; g v)} y^{\prime}\left(g ; \omega^{[2]}(g)\right)+x^{\prime}\left(g ; g r_{-}{ }^{[1]}\right) y^{\prime}\left(g ; g\left[r_{-}{ }^{[2]}, v\right]\right) \\
& +\Xi\left(\hat{L}_{x}(g), \hat{L}_{y}(g)\right)(v) \\
= & x^{\prime}\left(g ; \omega^{[1]}(g)\right) D_{(g ; g v)} y^{\prime}\left(g ; \omega^{[2]}(g)\right)+\hat{\Xi}\left(L_{x}(g), L_{y}(g)\right)(v) .
\end{aligned}
$$

We then convert back from $\tilde{\gamma}$ to $\gamma$ via (4-1).

For the remaining lemmas, as well as elsewhere, it is convenient to switch to an alternative notation for the bilinear functions $\Xi$ and $\hat{\Xi}$ from $\mathfrak{g}^{*} \otimes \mathfrak{g}^{*}$ to $\mathfrak{g}^{*}$. We can consider them as linear functions from $\mathfrak{g}$ to $\mathfrak{g} \otimes \mathfrak{g}$ with notation

$$
\Xi(\phi, \psi)(v)=(\phi \otimes \psi)(\Xi(v)), \quad \hat{\Xi}(\phi, \psi)(v)=(\phi \otimes \psi)(\hat{\Xi}(v))
$$


where $\Xi(v)=\Xi^{[1]}(v) \otimes \Xi^{[2]}(v)$ and $\hat{\Xi}(v)=\hat{\Xi}^{[1]}(v) \otimes \hat{\Xi}^{[2]}(v)$. Then we see that $\hat{\Xi}(v)=\Xi(v)+r_{-}{ }^{[1]} \otimes\left[r_{-}{ }^{[2]}, v\right]$.

Lemma 4.11. The commutator between a 2-form $\tilde{\tau}$ (expressed in the left trivialisation) and a function $y$ is given by

$$
\begin{aligned}
\tilde{\gamma}(y, \tilde{\tau}) & (g)(v, w) \\
& =y^{\prime}\left(g ; \omega^{[1]}(g)\right) \tilde{\tau}^{\prime}\left(g ; \omega^{[2]}(g)\right)+\left(\hat{L}_{y}(g) \otimes \tilde{\tau}(g)\right)(\Xi(v) \otimes w-\Xi(w) \otimes v) .
\end{aligned}
$$

Proof. Setting $\tau=\xi \wedge \eta$ for two 1-forms $\xi$ and $\eta$,

$$
\begin{aligned}
& \tilde{\gamma}(y, \tau)=\tilde{\gamma}(y, \xi) \wedge \eta+\xi \wedge \tilde{\gamma}(y, \eta) \\
& \tilde{\gamma}(y, \tau)(g)=y^{\prime}\left(g ; \omega^{[1]}(g)\right)\left(\xi^{\prime}\left(g ; \omega^{[2]}(g)\right) \wedge \eta(g)+\xi(g) \wedge \eta^{\prime}\left(g ; \omega^{[2]}(g)\right)\right) \\
& +\Xi\left(\hat{L}_{y}(g), \xi(g)\right) \wedge \eta(g)+\xi(g) \wedge \Xi\left(\hat{L}_{y}(g), \eta(g)\right) .
\end{aligned}
$$

Applying this to $(v, w)$ gives

$$
\begin{aligned}
& \tilde{\gamma}(y, \tau)(g)(v, w)=y^{\prime}\left(g ; \omega^{[1]}(g)\right) \tau^{\prime}\left(g ; \omega^{[2]}(g)\right)(v, w) \\
& \quad+\hat{L}_{y}(g)\left(\Xi^{[1]}(v)\right) \xi(g)\left(\Xi^{[2]}(v)\right) \eta(g)(w)-\hat{L}_{y}(g)\left(\Xi^{[1]}(w)\right) \xi(g)\left(\Xi^{[2]}(w)\right) \eta(g)(v) \\
& \quad+\xi(g)(v) \hat{L}_{y}(g)\left(\Xi^{[1]}(w)\right) \eta(g)\left(\Xi^{[2]}(w)\right)-\xi(g)(w) \hat{L}_{y}(g)\left(\Xi^{[1]}(v)\right) \eta(g)\left(\Xi^{[2]}(v)\right),
\end{aligned}
$$

which can be rearranged to give the answer.

Lemma 4.12. For a 1-form $\xi$ expressed in the left trivialisation, the $\mathrm{d}$ operation is given by

$$
\widetilde{\mathrm{d} \xi}(g)(v, w)=D_{(g ; g v)} \tilde{\xi}(g)(w)-D_{(g ; g w)} \tilde{\xi}(g)(v)+\tilde{\xi}(g)([w, v]) .
$$

Also we have

$$
\begin{aligned}
\mathrm{d} D_{(g ; X)} \tilde{\xi}(v, w) & -D_{(g ; X)} \mathrm{d} \tilde{\xi}(v, w) \\
& =\tilde{\xi}^{\prime}\left(g ; D_{(g ; g v)} X(g)-X v\right)(w)-\tilde{\xi}^{\prime}\left(g ; D_{(g ; g w)} X(g)-X w\right)(v) .
\end{aligned}
$$

Proof. By definition, $\mathrm{d} \xi(g)(g v, g w)=\xi^{\prime}(g ; g v)(g w)-\xi^{\prime}(g ; g w)(g v)$. However,

$$
D_{(g ; g v)} \tilde{\xi}(g)(w)=D_{(g ; g v)}(\xi(g)(g w))=\xi^{\prime}(g ; g v)(g w)+\xi(g)(g v w) .
$$

Lemma 4.13. The commutator between a 2-form $\widetilde{\mathrm{d} \xi}$ (expressed in the left trivialisation) and a function $y$ is given by

$$
\begin{aligned}
\tilde{\gamma}(x, \mathrm{~d} \tilde{\xi}) & (v, w)=x^{\prime}\left(g ; \omega^{[1]}(g)\right) \mathrm{d} D_{\left(g ; \omega^{[2]}(g)\right)} \tilde{\xi}(v, w) \\
& +\hat{L}_{x}\left(r_{-}{ }^{[1]}\right) \mathrm{d}\left(\operatorname{ad}_{r_{-}[2]}^{*}(\tilde{\xi})\right)(g)(v, w)+\left(\hat{L}_{x} \otimes \mathrm{d} \tilde{\xi}\right)(\hat{\Xi}(v) \otimes w-\hat{\Xi}(w) \otimes v) .
\end{aligned}
$$


Proof.

$$
\begin{aligned}
& \tilde{\gamma}(x, \mathrm{~d} \tilde{\xi})(v, w) \\
& =x^{\prime}\left(g ; \omega^{[1]}(g)\right) D_{\left(g ; \omega^{[2]}(g)\right)} \mathrm{d} \tilde{\xi}(v, w)+\left(\hat{L}_{x} \otimes \mathrm{d} \tilde{\xi}\right)(\Xi(v) \otimes w-\Xi(w) \otimes v) \\
& =x^{\prime}\left(g ; \omega^{[1]}(g)\right) \mathrm{d} D_{\left(g ; \omega^{[2]}(g)\right)} \tilde{\xi}(v, w)-x^{\prime}\left(g ; \omega^{[1]}(g)\right) \tilde{\xi}^{\prime}\left(g ; D_{(g ; g v)} \omega^{[2]}(g)\right. \\
& \left.-\omega^{[2]}(g) v\right)(w)+x^{\prime}\left(g ; \omega^{[1]}(g)\right) \tilde{\xi}^{\prime}\left(g ; D_{(g ; g w)} \omega^{[2]}(g)-\omega^{[2]}(g) w\right)(v) \\
& +\left(\hat{L}_{x} \otimes \mathrm{d} \tilde{\xi}\right)(\Xi(v) \otimes w-\Xi(w) \otimes v) \\
& =x^{\prime}\left(g ; \omega^{[1]}(g)\right) \mathrm{d} D_{\left(g ; \omega^{[2]}(g)\right)} \tilde{\xi}(v, w)-\hat{L}_{x}\left(r_{-}{ }^{[1]}\right) \tilde{\xi}^{\prime}\left(g ; g\left[v, r_{-}{ }^{[2]}\right]\right)(w) \\
& +\hat{L}_{x}\left(r_{-}{ }^{[1]}\right) \tilde{\xi}^{\prime}\left(g ; g\left[w, r_{-}{ }^{[2]}\right]\right)(v)+\left(\hat{L}_{x} \otimes \mathrm{d} \tilde{\xi}\right)(\Xi(v) \otimes w-\Xi(w) \otimes v) \\
& =x^{\prime}\left(g ; \omega^{[1]}(g)\right) \mathrm{d} D_{\left(g ; \omega^{[2]}(g)\right)} \tilde{\xi}(v, w)-\hat{L}_{x}\left(r_{-}{ }^{[1]}\right) \tilde{\xi}^{\prime}\left(g ; g\left[v, r_{-}{ }^{[2]}\right]\right)(w) \\
& +\hat{L}_{x}\left(r_{-}{ }^{[1]}\right) \tilde{\xi}^{\prime}\left(g ; g\left[w, r_{-}{ }^{[2]}\right]\right)(v)+\left(\hat{L}_{x} \otimes \mathrm{d} \tilde{\xi}\right)(\hat{\Xi}(v) \otimes w-\hat{\Xi}(w) \otimes v) \\
& +\hat{L}_{x}\left(r_{-}{ }^{[1]}\right) \mathrm{d} \tilde{\xi}\left(\left[v, r_{-}{ }^{[2]}\right], w\right)-\hat{L}_{x}\left(r_{-}{ }^{[1]}\right) \mathrm{d} \tilde{\xi}\left(\left[w, r_{-}{ }^{[2]}\right], v\right) \\
& =x^{\prime}\left(g ; \omega^{[1]}(g)\right) \mathrm{d} D_{\left(g ; \omega^{[2]}(g)\right)} \tilde{\xi}(v, w)-\hat{L}_{x}\left(r_{-}{ }^{[1]}\right) \tilde{\xi}^{\prime}(g ; g w)\left(\left[v, r_{-}{ }^{[2]}\right]\right) \\
& +\hat{L}_{x}\left(r_{-}{ }^{[1]}\right) \tilde{\xi}^{\prime}(g ; g v)\left(\left[w, r_{-}{ }^{[2]}\right]\right)+\left(\hat{L}_{x} \otimes \mathrm{d} \tilde{\xi}\right)(\hat{\Xi}(v) \otimes w-\hat{\Xi}(w) \otimes v) \\
& -\hat{L}_{x}\left(r_{-}{ }^{[1]}\right) \tilde{\xi}(g)\left(\left[\left[v, r_{-}{ }^{[2]}\right], w\right]-\left[\left[w, r_{-}{ }^{[2]}\right], v\right]\right) \\
& =x^{\prime}\left(g ; \omega^{[1]}(g)\right) \mathrm{d} D_{\left(g ; \omega^{[2]}(g)\right)} \tilde{\xi}(v, w)+\hat{L}_{x}\left(r_{-}{ }^{[1]}\right) \mathrm{d}\left(\operatorname{ad}_{r_{-}{ }^{[2]}}(\tilde{\xi})\right)(g)(v, w) \\
& +\left(\hat{L}_{x} \otimes \mathrm{d} \tilde{\xi}\right)(\hat{\Xi}(v) \otimes w-\hat{\Xi}(w) \otimes v) .
\end{aligned}
$$

4C. Example: quantum $\mathrm{SU}_{2}$ with its left-covariant calculus. Here we pause to compute a quantum group example, namely the 3-dimensional calculus on $C_{\hbar}\left(\mathrm{SU}_{2}\right)$ found in [Woronowicz 1989]. As with our previous example, we do not need the full $C^{*}$ theory but just a subalgebra generated by a matrix of generators $\left(\begin{array}{ll}a & b \\ c & d\end{array}\right)$, which in the classical limit become the matrix element coordinate functions on $\mathrm{SU}_{2}$. We use the standard relations

$b a=q a b, \quad c a=q a c, \quad d b=q b d, \quad d c=q c d, \quad c b=b c$,

$$
a d=1+q^{-1} b c, \quad d a=1+q b c .
$$

For our purposes $q=e^{\frac{\hbar}{2}}$ rather than $q$ real as needed for completion to a $C^{*}$ algebra. For the rest of the Hopf algebra structure we refer to any textbook on quantum groups. The 3 -d calculus has a basis of left-invariant 1-forms

$$
\tau^{+}=d \mathrm{~d} b-q b \mathrm{~d} d, \quad \tau^{-}=q a \mathrm{~d} c-c \mathrm{~d} a, \quad \tau^{3}=d \mathrm{~d} a-q b \mathrm{~d} c
$$

with commutation relations

$$
\tau^{ \pm}\left(\begin{array}{ll}
a & b \\
c & d
\end{array}\right)=\left(\begin{array}{ll}
q a & q^{-1} b \\
q c & q^{-1} d
\end{array}\right) \tau^{ \pm}, \quad \tau^{3}\left(\begin{array}{ll}
a & b \\
c & d
\end{array}\right)=\left(\begin{array}{ll}
q^{2} a & q^{-2} b \\
q^{2} c & q^{-2} d
\end{array}\right) \tau^{3} .
$$


One has

$$
\begin{array}{ll}
\mathrm{d} a=a \tau^{3}+q^{-1} b \tau^{-}, & \mathrm{d} b=a \tau^{+}-q^{-2} b \tau^{3}, \\
\mathrm{~d} c=c \tau^{3}+q^{-1} d \tau^{-}, & \mathrm{d} d=c \tau^{+}-q^{-2} d \tau^{3} .
\end{array}
$$

The Poisson bracket can be read off from the algebra relations as generated by the nonzero values $\{a, b\}=-\frac{1}{2} a b, \quad\{a, c\}=-\frac{1}{2} a c, \quad\{a, d\}=-b c, \quad\{b, d\}=-\frac{1}{2} b d, \quad\{c, d\}=-\frac{1}{2} c d$. Classically the $\tau^{ \pm}, \tau^{3}$ are dual to the left invariant vector fields $\partial_{ \pm}, \partial_{3}$ generated by the Lie algebra $\mathfrak{s u}_{2}$ and

$$
\mathrm{d} x=\left(\partial_{i} x\right) \tau^{i},
$$

where the sum is over $i= \pm, 3$. Explicitly here,

$$
\partial_{+}\left(\begin{array}{ll}
a & b \\
c & d
\end{array}\right)=\left(\begin{array}{ll}
0 & a \\
0 & c
\end{array}\right), \quad \partial_{-}\left(\begin{array}{ll}
a & b \\
c & d
\end{array}\right)=\left(\begin{array}{ll}
b & 0 \\
d & 0
\end{array}\right), \quad \partial_{3}\left(\begin{array}{ll}
a & b \\
c & d
\end{array}\right)=\left(\begin{array}{ll}
a & -b \\
c & -d
\end{array}\right)
$$

computed by right multiplication in the usual representation of the Chevalley basis of $\mathfrak{s u}_{2}$. Meanwhile, from the bimodule commutation relations we read off

$$
\gamma\left(\left(\begin{array}{ll}
a & b \\
c & d
\end{array}\right), \tau^{i}\right)=\frac{1}{2} \lambda_{i}\left(\begin{array}{ll}
a & -b \\
c & -d
\end{array}\right) \tau^{i}, \quad \lambda_{ \pm}=-1, \quad \lambda_{3}=-2
$$

and since $\gamma\left(, \tau^{i}\right)$ acts as derivation, comparing values on these generators, one finds

$$
\gamma\left(x, \tau^{i}\right)=\frac{1}{2} \lambda_{i}\left(\partial_{3} x\right) \tau^{i}
$$

for all functions $x$. Hence for all $\eta=\eta_{i} \tau^{i}$,

$$
\hat{\nabla}_{x}(\eta)=\gamma(x, \eta)=\left\{x, \eta_{i}\right\} \tau^{i}+\frac{1}{2} \lambda_{i}\left(\partial_{3} x\right) \eta_{i} \tau^{i} .
$$

From this and the compatibility condition (2-5) one may verify that the curvature $R(x, y)\left(\tau^{i}\right)=0$ and hence $R(x, y)=0$ as it must since $\Omega^{1}$ for the quantum calculus is a bimodule. We are not in the symplectic case so the torsion need not vanish.

We can understand this example using our above results as follows. The standard quasitriangular structure is

$$
r=e_{+} \otimes e_{-}+\frac{1}{4} e_{3} \otimes e_{3}
$$

in the Chevalley basis (so that $\left[e_{3}, e_{ \pm}\right]= \pm 2 e_{ \pm}$and $\left[e_{+}, e_{-}\right]=e_{3}$ ) and one may check that this reproduces the above Poisson-bracket with $\omega=g r-r g$. This is the usual picture for $\mathrm{SU}_{2}$ with its standard Drinfeld-Sklyanin Poisson bracket.

Next, the Lie cobracket is

$$
\delta e_{i}=\frac{1}{2}\left(e_{i} \otimes e_{3}-e_{3} \otimes e_{i}\right),
$$


so

$$
\Xi\left(e_{i}\right)=\frac{1}{2} \lambda_{i} e_{3} \otimes e_{i}, \quad \lambda_{ \pm}=-1 .
$$

We will solve the condition in Proposition 4.6 for any $\lambda_{3}$, giving a compatible preconnection. In the left trivialisation, suppose (as above) that $\tilde{\tau}^{i} \in \mathfrak{g}^{*}$ are constant and dual to the basis $e_{i}$. Then

$$
\tilde{\gamma}\left(a, \tilde{\tau}^{i}\right)=\Xi\left(\hat{L}_{a}(g), \tilde{\tau}^{i}\right)=\tilde{\tau}^{j} a^{\prime}\left(g ; g \Xi^{[1]}\left(e_{j}\right)\right)\left\langle\Xi^{[2]}\left(e_{j}\right), \tilde{\tau}^{i}\right\rangle=\frac{1}{2} \lambda_{i}\left(\partial_{3} a\right) \tilde{\tau}^{i} .
$$

Since $\tau^{i}$ are the left invariant extensions of $\tilde{\tau}^{i}$ (the Maurer-Cartan form) we have exactly (4-5) except that for us $\lambda_{3}$ is arbitrary. For this form of connection the compatibility condition reduces to $\partial_{i}\{x, y\}=\left\{\partial_{i} x, y\right\}+\left\{x, \partial_{i} y\right\}$ which holds as it must. The curvature $R$ then vanishes by this equation, for all $\lambda_{3}$. There is a similar natural form for $\Xi$ for all simple $\mathfrak{g}$ with their standard Poisson-Lie group structures according to the form of $\delta$.

The Poisson tensor is

$$
c b\left(\partial_{-} \otimes \partial_{+}-\partial_{+} \otimes \partial_{-}\right)+\frac{b d}{2}\left(\partial_{+} \otimes \partial_{3}-\partial_{3} \otimes \partial_{+}\right)+\frac{c a}{2}\left(\partial_{-} \otimes \partial_{3}-\partial_{3} \otimes \partial_{-}\right) .
$$

Now (summing over repeated indices), (4-4) and (4-6) give

$$
\hat{\nabla}_{x}(\mathrm{~d} z)=\left\{x, \partial_{i} z\right\} \tau^{i}+\frac{1}{2} \lambda_{i}\left(\partial_{3} x\right)\left(\partial_{i} z\right) \tau^{i},
$$

so we get

$$
\begin{aligned}
\left\langle\hat{y}, \hat{\nabla}_{x}(\mathrm{~d} z)\right\rangle & =\omega^{j i}\left(\partial_{j} y\right)\left(\left\{x, \partial_{i} z\right\}+\frac{1}{2} \lambda_{i}\left(\partial_{3} x\right)\left(\partial_{i} z\right)\right) \\
& =\omega^{j i} \omega^{n m}\left(\partial_{j} y\right)\left(\partial_{n} x\right)\left(\partial_{m} \partial_{i} z\right)+\frac{1}{2} \lambda_{i} \omega^{j i}\left(\partial_{j} y\right)\left(\partial_{3} x\right)\left(\partial_{i} z\right) .
\end{aligned}
$$

Then from (3-3) we can write the torsion as

$$
\langle d z, T(x, y)\rangle=\frac{1}{2} \lambda_{i} \omega^{j i}\left(\partial_{j} y\right)\left(\partial_{3} x\right)\left(\partial_{i} z\right)-\frac{1}{2} \lambda_{i} \omega^{j i}\left(\partial_{j} x\right)\left(\partial_{3} y\right)\left(\partial_{i} z\right),
$$

which is nonzero.

4D. Bicovariant calculi on Poisson-Lie groups. We now assume that the differential calculus is bicovariant, resulting in important simplifications and our main results.

Theorem 4.14. A left invariant preconnection $\tilde{\gamma}$ given by $\Xi$ is bicovariant if, for all $g \in G$ and $\phi, \psi \in \mathfrak{g}^{*}$,

$$
\Xi(\phi, \psi)-\operatorname{Ad}_{g^{-1}}^{*} \Xi\left(\operatorname{Ad}_{g}^{*} \phi, \operatorname{Ad}_{g}^{*} \psi\right)=\phi\left(g^{-1} \omega^{[1]}(g)\right) \operatorname{ad}_{g^{-1} \omega^{[2]}(g)}^{*} \psi .
$$

Proof. The section $t$ of the trivial $\mathfrak{g}^{*}$ bundle given by trivialising $T^{*} G$ by the right action is related to the section $s$ of the left trivialisation by $t(g)=\left(\operatorname{Ad}_{g^{-1}}\right)^{*} s(g)$, and the corresponding right invariant connection would be, for some $\Psi: \mathfrak{g}^{*} \otimes \mathfrak{g}^{*} \rightarrow \mathfrak{g}^{*}$,

$$
\left(\operatorname{Ad}_{g^{-1}}\right)^{*} \tilde{\gamma}(y, s)(g)=\{y, t\}+\Psi\left(\hat{R}_{y}(g), t(g)\right) .
$$


Here we have $\hat{R}_{y}(g)=\left(\operatorname{Ad}_{g-1}\right)^{*}\left(\hat{L}_{y}(g)\right)$, as can be seen from

$$
\hat{R}_{y}(g)(v)=y^{\prime}(g ; v g)=y^{\prime}\left(g ; g \operatorname{Ad}_{g^{-1}} v\right) .
$$

From now we will use the coadjoint action, $\operatorname{Ad}_{g}^{*}=\left(\operatorname{Ad}_{g-1}\right)^{*}$. Then

$$
t^{\prime}(g ; v)=\operatorname{Ad}_{g}^{*}\left(\operatorname{ad}_{g^{-1} v}^{*} s(g)+s^{\prime}(g ; v)\right)
$$

so (4-7) becomes

$$
\begin{aligned}
\tilde{\gamma}(y, s)(g)=y^{\prime}\left(g ; \omega^{[1]}(g)\right)\left(\operatorname{ad}_{g^{-1} \omega^{[2]}(g)}^{*} s(g)+s^{\prime}\right. & \left.\left(g ; \omega^{[2]}(g)\right)\right) \\
& +\operatorname{Ad}_{g^{-1}}^{*} \Psi\left(\operatorname{Ad}_{g}^{*} \hat{L}_{y}(g), \operatorname{Ad}_{g}^{*} s(g)\right) .
\end{aligned}
$$

Substituting Definition 4.4 into this gives

$$
\Xi\left(\hat{L}_{y}(g), s(g)\right)-\operatorname{Ad}_{g^{-1}}^{*} \Psi\left(\operatorname{Ad}_{g}^{*} \hat{L}_{y}(g), \operatorname{Ad}_{g}^{*} s(g)\right)=y^{\prime}\left(g ; \omega^{[1]}(g)\right) \operatorname{ad}_{g^{-1} \omega^{[2]}(g)}^{*} s(g) .
$$

We can use the definition of $\hat{L}_{y}(g)$ to rewrite this as

$$
\begin{aligned}
& \Xi\left(\hat{L}_{y}(g), s(g)\right)-\operatorname{Ad}_{g^{-1}}^{*} \Psi\left(\operatorname{Ad}_{g}^{*} \hat{L}_{y}(g), \operatorname{Ad}_{g}^{*} s(g)\right) \\
& \quad=\hat{L}_{y}(g)\left(g^{-1} \omega^{[1]}(g)\right) \operatorname{ad}_{g^{-1} \omega^{[2]}(g)}^{*} s(g) .
\end{aligned}
$$

Now we set $\phi=\hat{L}_{y}(g) \in \mathfrak{g}^{*}$ and $\psi=s(g) \in \mathfrak{g}^{*}$,

$$
\Xi(\phi, \psi)-\operatorname{Ad}_{g^{-1}}^{*} \Psi\left(\operatorname{Ad}_{g}^{*} \phi, \operatorname{Ad}_{g}^{*} \psi\right)=\phi\left(g^{-1} \omega^{[1]}(g)\right) \operatorname{ad}_{g^{-1} \omega^{[2]}(g)}^{*} \psi .
$$

Setting $g=e$ now tells us that $\Xi=\Psi$.

Proposition 4.15. In the case of $\mathfrak{g}$ quasitriangular, a left-invariant preconnection $\tilde{\gamma}$ given by $\hat{\Xi}$ is bicovariant if and only if $\hat{\Xi}$ is Ad-invariant. The infinitesimal form of this is

$$
\hat{\Xi}([v, w])=\left[\hat{\Xi}^{[1]}(v), w\right] \otimes \hat{\Xi}^{[2]}(v)+\hat{\Xi}^{[1]}(v) \otimes\left[\hat{\Xi}^{[2]}(v), w\right] .
$$

Proof. Use Theorem 4.14 and $\omega(g)=g r_{-}-r_{-} g$. Ad-invariance means of course $\hat{\Xi}(\phi, \psi)=\operatorname{Ad}_{g^{-1}}^{*} \hat{\Xi}\left(\operatorname{Ad}_{g}^{*} \phi, \operatorname{Ad}_{g}^{*} \psi\right)$ for all $g \in G$, which we then write as stated.

Proposition 4.16. In the quasitriangular bicovariant case, the first super-Jacobi identity corresponds to the vanishing of

$$
(\mathrm{id} \otimes \hat{\Xi}) \hat{\Xi}(v)-(\tau \otimes \mathrm{id})(\mathrm{id} \otimes \hat{\Xi}) \hat{\Xi}(v)+n^{[1]} \otimes n^{[2]} \otimes\left[v, n^{[3]}\right]
$$

where $n=\llbracket r_{-}, r_{-} \rrbracket$ and $\tau$ is the usual flip map.

Proof. We use the result of Lemma 4.9 and $\hat{\Xi}$ now ad-invariant.

In other words, this expression corresponds to the curvature of the preconnection in the geometric picture of Section 3. The second Jacobi identity is much more painful but turn out to also be controlled by this element $n$ : 
Proposition 4.17. In the quasitriangular bicovariant case if the first super-Jacobi identity holds, the second super-Jacobi identity corresponds to the vanishing of

$$
\begin{aligned}
2 & D_{(g ; g w)}\left(\hat{L}_{x}\left(e^{[1]}(v)\right)\right) \hat{L}_{x}\left(e^{[2]}(v)\right) \hat{L}_{x}\left(e^{[3]}(v)\right) \\
& -2 D_{(g ; g v)}\left(\hat{L}_{x}\left(e^{[1]}(w)\right)\right) \hat{L}_{x}\left(e^{[2]}(w)\right) \hat{L}_{x}\left(e^{[3]}(w)\right) \\
& +\hat{L}_{x}\left(\left[e^{[1]}(v), w\right]\right) \hat{L}_{x}\left(e^{[2]}(v)\right) \hat{L}_{x}\left(e^{[3]}(v)\right)-\hat{L}_{x}\left(\left[e^{[1]}(w), v\right]\right) \hat{L}_{x}\left(e^{[2]}(w)\right) \hat{L}_{x}\left(e^{[3]}(w)\right)
\end{aligned}
$$

for all $x$ and $v, w \in \mathfrak{g}$, where $e(v)=-n^{[1]} \otimes n^{[2]} \otimes\left[v, n^{[3]}\right]$.

Proof. For brevity we write Lemma 4.10 as

$$
\begin{aligned}
{[y, \mathrm{~d} x]_{\bullet} } & =\hbar\left(p_{y}^{1} \mathrm{~d} p_{x}^{2}+h_{y x}\right)+O\left(\hbar^{2}\right), \\
{[\mathrm{d} y, \mathrm{~d} x]_{\bullet} } & =\hbar\left(\mathrm{d} p_{y}^{1} \wedge \mathrm{d} p_{x}^{2}+\mathrm{d} h_{y x}\right)+O\left(\hbar^{2}\right),
\end{aligned}
$$

where $p_{y}^{1}(g)=y^{\prime}\left(g ; \omega^{[1]}(g)\right), p_{x}^{2}(g)=x^{\prime}\left(g ; \omega^{[2]}(g)\right)$,

$$
h_{y x}(g ; g v)=\hat{\Xi}\left(\hat{L}_{y}(g), \hat{L}_{x}(g)\right)(v),
$$

and the second commutator result is just the differential of the first. Then

$$
\begin{aligned}
{\left[z,[\mathrm{~d} y, \mathrm{~d} x]_{\bullet}\right]_{\bullet} } & =\hbar\left(\left[z, \mathrm{~d} p_{y}^{1}\right]_{\bullet} \wedge \mathrm{d} p_{x}^{2}+\mathrm{d} p_{y}^{1} \wedge\left[z, \mathrm{~d} p_{x}^{2}\right]_{\bullet}+\left[z, \mathrm{~d} h_{y x}\right]_{\bullet}\right)+O\left(\hbar^{3}\right) \\
& =\hbar\left(\left[z, \mathrm{~d} p_{y}^{1}\right]_{\bullet} \wedge \mathrm{d} p_{x}^{2}+\left[z, \mathrm{~d} p_{x}^{1}\right]_{\bullet} \wedge \mathrm{d} p_{y}^{2}+\left[z, \mathrm{~d} h_{y x}\right]_{\bullet}\right)+O\left(\hbar^{3}\right), \\
{\left[\mathrm{d} x,[z, \mathrm{~d} y]_{\bullet}\right]_{\bullet} } & =\hbar\left(\left[\mathrm{d} x, p_{z}^{1} \mathrm{~d} p_{y}^{2}+h_{z y}\right]_{\bullet}\right)+O\left(\hbar^{3}\right) \\
& =\hbar\left(-\left[p_{z}^{1}, \mathrm{~d} x\right]_{\bullet} \wedge \mathrm{d} p_{y}^{2}+p_{z}^{1}\left[\mathrm{~d} x, \mathrm{~d} p_{y}^{2}\right]_{\bullet}+\left[\mathrm{d} x, h_{z y}\right]_{\bullet}\right)+O\left(\hbar^{3}\right) .
\end{aligned}
$$

Now we can calculate

$$
\begin{aligned}
& \hbar J_{2}(x, x, x)+O\left(\hbar^{2}\right) \\
& \quad=\hbar^{-1}\left(\left[x,[\mathrm{~d} x, \mathrm{~d} x]_{\bullet}\right]_{\bullet}-\left[\mathrm{d} x,[x, \mathrm{~d} x]_{\bullet}\right]_{\bullet}-\left[\mathrm{d} x,[x, \mathrm{~d} x]_{\bullet}\right]_{\bullet}\right) \\
& \quad=2\left(\left[x, \mathrm{~d} p_{x}^{1}\right]_{\bullet}+\left[p_{x}^{1}, \mathrm{~d} x\right]_{\bullet}\right) \wedge \mathrm{d} p_{x}^{2}-2 p_{x}^{1}\left[\mathrm{~d} x, \mathrm{~d} p_{x}^{2}\right]_{\bullet}-2\left[\mathrm{~d} x, h_{x x}\right]_{\bullet}+\left[x, \mathrm{~d} h_{x x}\right]_{\bullet} .
\end{aligned}
$$

Now we can use an independent copy $q$ of $p$ to obtain

$$
\begin{aligned}
{\left[p_{x}^{1}, \mathrm{~d} x\right]_{\bullet} } & =\hbar\left(q_{p_{x}^{1}}^{1} \mathrm{~d} q_{x}^{2}+h_{p_{x}^{1} x}\right)+O\left(\hbar^{2}\right), \\
{\left[x, \mathrm{~d} p_{x}^{1}\right]_{\bullet} } & =\hbar\left(q_{x}^{1} \mathrm{~d} q_{p_{x}^{1}}^{2}+h_{p_{x}^{1} x}\right)+O\left(\hbar^{2}\right), \\
{\left[\mathrm{d} q_{x}^{2}, \mathrm{~d} x\right]_{\bullet} } & =\hbar\left(\mathrm{d} p_{q_{x}^{2}}^{1} \wedge \mathrm{d} p_{x}^{2}+\mathrm{d} h_{q_{x}^{2} x}\right)+O\left(\hbar^{2}\right) .
\end{aligned}
$$

From this we get

$$
\begin{aligned}
J_{2}(x, x, x)=2\left(q_{p_{x}^{1}}^{1} \mathrm{~d} q_{x}^{2}+\right. & \left.2 h_{p_{x}^{1} x}+q_{x}^{1} \mathrm{~d} q_{p_{x}^{1}}^{2}-q_{x}^{1} \mathrm{~d} p_{q_{x}^{2}}^{1}\right) \wedge \mathrm{d} p_{x}^{2} \\
& -2 p_{x}^{1} \mathrm{~d} h_{p_{x}^{2} x}-2\left[\mathrm{~d} x, h_{x x}\right]_{\bullet} / \hbar+\left[x, \mathrm{~d} h_{x x}\right]_{\bullet} / \hbar+O(\hbar) .
\end{aligned}
$$


Suppose that $p$ corresponds to $\omega$ and $q$ to $\pi$, another copy of $\omega$. Then

$$
\begin{aligned}
q_{p_{x}^{1}}^{2}-p_{q_{x}^{2}}^{1} & =x^{\prime}\left(g ; D_{\left(g ; \pi^{2}(g)\right)} \omega^{[1]}(g)-D_{\left(g ; \omega^{[1]}(g)\right)} \pi^{2}(g)\right), \\
q_{p_{x}^{1}}^{1} \mathrm{~d} q_{x}^{2} \wedge \mathrm{d} p_{x}^{2} & =p_{q_{x}^{1}}^{1} \mathrm{~d} p_{x}^{2} \wedge \mathrm{d} q_{x}^{2}=-p_{q_{x}^{1}}^{1} \mathrm{~d} q_{x}^{2} \wedge \mathrm{d} p_{x}^{2}, \\
2 q_{p_{x}^{1}}^{1} \mathrm{~d} q_{x}^{2} \wedge \mathrm{d} p_{x}^{2} & =\left(q_{p_{x}^{1}}^{1}-p_{q_{x}^{1}}^{1}\right) \mathrm{d} q_{x}^{2} \wedge \mathrm{d} p_{x}^{2} \\
& =x^{\prime}\left(g ; D_{\left(g ; \pi^{1}(g)\right)} \omega^{[1]}(g)-D_{\left(g ; \omega^{[1]}(g)\right)} \pi^{1}(g)\right) \mathrm{d} q_{x}^{2} \wedge \mathrm{d} p_{x}^{2} .
\end{aligned}
$$

For $t$ an independent copy of $r$, we have

$$
\begin{aligned}
2 q_{p_{x}^{1}}^{1} \mathrm{~d} q_{x}^{2} \wedge \mathrm{d} p_{x}^{2}=x^{\prime}\left(g ; g\left[t_{-}{ }^{[1]}\right.\right. & \left.\left., r_{-}{ }^{[1]}\right]\right) \mathrm{d} x^{\prime}\left(g ; g t_{-}{ }^{[2]}\right) \wedge \mathrm{d} x^{\prime}\left(g ; g r_{-}{ }^{[2]}\right) \\
& -x^{\prime}\left(g ;\left[t_{-}{ }^{[1]}, r_{-}{ }^{[1]}\right] g\right) \mathrm{d} x^{\prime}\left(g ; t_{-}{ }^{[2]} g\right) \wedge \mathrm{d} x^{\prime}\left(g ; r_{-}{ }^{[2]} g\right)
\end{aligned}
$$

and

$$
\begin{aligned}
q_{x}^{1} \mathrm{~d}\left(q_{p_{x}^{1}}^{2}-p_{q_{x}^{2}}^{1}\right) \wedge \mathrm{d} p_{x}^{2}=x^{\prime}\left(g ; g t_{-}{ }^{[1]}\right) \mathrm{d} x^{\prime}\left(g ; g\left[t_{-}{ }^{[2]}, r_{-}{ }^{[1]}\right]\right) \wedge \mathrm{d} x^{\prime}\left(g ; g r_{-}{ }^{[2]}\right) \\
-x^{\prime}\left(g ; t_{-}{ }^{[1]} g\right) \mathrm{d} x^{\prime}\left(g ;\left[t_{-}{ }^{[2]}, r_{-}{ }^{[1]}\right] g\right) \wedge \mathrm{d} x^{\prime}\left(g ; r_{-}{ }^{[2]} g\right) \\
=-x^{\prime}\left(g ; g t_{-}{ }^{[1]}\right) \mathrm{d} x^{\prime}\left(g ; g r_{-}{ }^{[2]}\right) \wedge \mathrm{d} x^{\prime}\left(g ; g\left[t_{-}{ }^{[2]}, r_{-}{ }^{[1]}\right]\right) \\
+x^{\prime}\left(g ; t_{-}{ }^{[1]} g\right) \mathrm{d} x^{\prime}\left(g ; r_{-}{ }^{[2]} g\right) \wedge \mathrm{d} x^{\prime}\left(g ;\left[t_{-}{ }^{[2]}, r_{-}{ }^{[1]}\right] g\right) \\
=+x^{\prime}\left(g ; g t_{-}{ }^{[1]}\right) \mathrm{d} x^{\prime}\left(g ; g r_{-}{ }^{[1]}\right) \wedge \mathrm{d} x^{\prime}\left(g ; g\left[t_{-}{ }^{[2]}, r_{-}{ }^{[2]}\right]\right) \\
\\
\quad-x^{\prime}\left(g ; t_{-}{ }^{[1]} g\right) \mathrm{d} x^{\prime}\left(g ; r_{-}{ }^{[1]} g\right) \wedge \mathrm{d} x^{\prime}\left(g ;\left[t_{-}{ }^{[2]}, r_{-}{ }^{[2]}\right] g\right) .
\end{aligned}
$$

Using this,

$$
\begin{aligned}
2\left(q_{p_{x}^{1}}^{1} \mathrm{~d} q_{x}^{2}+q_{x}^{1} \mathrm{~d} q_{p_{x}^{1}}^{2}-q_{x}^{1} \mathrm{~d} p_{q_{x}^{2}}^{1}\right) \wedge \mathrm{d} p_{x}^{2}= & +x^{\prime}\left(g ; g n^{[1]}\right) \mathrm{d} x^{\prime}\left(g ; g n^{[2]}\right) \wedge \mathrm{d} x^{\prime}\left(g ; g n^{[3]}\right) \\
& -x^{\prime}\left(g ; n^{[1]} g\right) \mathrm{d} x^{\prime}\left(g ; n^{[2]} g\right) \wedge \mathrm{d} x^{\prime}\left(g ; n^{[3]} g\right),
\end{aligned}
$$

where $n=\llbracket r_{-}, r_{-} \rrbracket$. As $n$ is Ad-invariant, we have shown that

$$
\begin{aligned}
J_{2}(x, x, x) & =4 h_{p_{x}^{1} x} \wedge \mathrm{d} p_{x}^{2}-2 p_{x}^{1} \mathrm{~d} h_{p_{x}^{2} x}-2\left[\mathrm{~d} x, h_{x x}\right]_{\bullet} / \hbar+\left[x, \mathrm{~d} h_{x x}\right]_{\bullet} / \hbar+O(\hbar) \\
& =4 h_{p_{x}^{1} x} \wedge \mathrm{d} p_{x}^{2}+2 p_{x}^{2} \mathrm{~d} h_{p_{x}^{1} x}-2 \mathrm{~d}\left[x, h_{x x}\right]_{\bullet} / \hbar+3\left[x, \mathrm{~d} h_{x x}\right]_{\bullet} / \hbar+O(\hbar) .
\end{aligned}
$$

Next, using the equation

$$
D_{\left(g ; \omega^{[2]}(g)\right)} \hat{L}_{x}(v)-\hat{L}_{p_{x}^{2}}(v)=x^{\prime}\left(g ; \omega^{[2]}(g) v-D_{(g ; g v)} \omega^{[2]}(g)\right),
$$

we can write

$$
\begin{aligned}
h_{x x}^{\prime}\left(g ; \omega^{[2]}(g)\right) & =2 \hat{\Xi}\left(D_{\left(g ; \omega^{[2]}(g)\right)} \hat{L}_{x}, \hat{L}_{x}\right) \\
& =2 h_{p_{x}^{2} x}+2 \hat{\Xi}\left(v \mapsto x^{\prime}\left(g ; \omega^{[2]}(g) v-D_{(g ; g v)} \omega^{[2]}(g)\right), \hat{L}_{x}\right)
\end{aligned}
$$


and

$$
\begin{aligned}
p_{y}^{1}(g) h_{x x}^{\prime}\left(g ; \omega^{[2]}(g)\right) & \\
& =2 p_{y}^{1}(g) h_{p_{x}^{2} x}(g)+2 y^{\prime}\left(g ; g r_{-}{ }^{[1]}\right) \hat{\Xi}\left(v \mapsto x^{\prime}\left(g ; g\left[r_{-}{ }^{[2]}, v\right]\right), \hat{L}_{x}\right) \\
& =2 p_{y}^{1}(g) h_{p_{x}^{2} x}(g)-2 \hat{L}_{y}(g)\left(r_{-}{ }^{[1]}\right) \hat{\Xi}\left(\operatorname{ad}_{r_{-}}^{*}{ }^{[2]} \hat{L}_{x}, \hat{L}_{x}\right) .
\end{aligned}
$$

Then, using the ad-invariance of $\hat{\Xi}$,

$$
\begin{aligned}
{\left[y, h_{x x}\right]_{\bullet} / \hbar=} & x^{\prime}\left(g ; \omega^{[1]}(g)\right) h_{x x}^{\prime}\left(g ; \omega^{[2]}(g)\right)+\Xi\left(\hat{L}_{x}, h_{x x}\right)+O(\hbar) \\
= & 2 p_{y}^{1}(g) h_{p_{x}^{2} x}(g)+\hat{\Xi}\left(\hat{L}_{y}, h_{x x}\right) \\
& \quad+\hat{L}_{y}(g)\left(r_{-}{ }^{[1]}\right)\left(\operatorname{ad}_{r_{-}[2]}^{*} \hat{\Xi}\left(\hat{L}_{x}, \hat{L}_{x}\right)-2 \hat{\Xi}\left(\operatorname{ad}_{r_{-}[2]}^{*} \hat{L}_{x}, \hat{L}_{x}\right)\right)+O(\hbar) \\
= & 2 p_{y}^{1}(g) h_{p_{x}^{2} x}(g)+\hat{\Xi}\left(\hat{L}_{y}, h_{x x}\right)+O(\hbar) .
\end{aligned}
$$

This gives

$$
\begin{gathered}
J_{2}(x, x, x)=4 h_{p_{x}^{1} x} \wedge \mathrm{d} p_{x}^{2}+2 p_{x}^{2} \mathrm{~d} h_{p_{x}^{1} x}-2 \mathrm{~d}\left(2 p_{x}^{1}(g) h_{p_{x}^{2} x}(g)+\hat{\Xi}\left(\hat{L}_{x}, h_{x x}\right)\right) \\
+3\left[x, \mathrm{~d} h_{x x}\right]_{\bullet} / \hbar+O(\hbar) \\
=4 h_{p_{x}^{1} x} \wedge \mathrm{d} p_{x}^{2}+2 p_{x}^{2} \mathrm{~d} h_{p_{x}^{1} x}+4 \mathrm{~d}\left(p_{x}^{2}(g) h_{p_{x}^{1} x}(g)\right) \\
-2 \mathrm{~d} \hat{\Xi}\left(\hat{L}_{x}, h_{x x}\right)+3\left[x, \mathrm{~d} h_{x x}\right]_{\bullet} / \hbar+O(\hbar) \\
=6 p_{x}^{2} \mathrm{~d} h_{p_{x}^{1} x}-2 \mathrm{~d} \hat{\Xi}\left(\hat{L}_{x}, h_{x x}\right)+3\left[x, \mathrm{~d} h_{x x}\right]_{\bullet} / \hbar+O(\hbar) .
\end{gathered}
$$

Next, setting

$$
E=\hat{L}_{x}(g)\left(r_{-}{ }^{[1]}\right) \hat{\Xi}\left(\operatorname{ad}_{r_{-}[2]}^{*} \hat{L}_{x}, \hat{L}_{x}\right),
$$

we find

$$
p_{x}^{1}(g) h_{x x}^{\prime}\left(g ; \omega^{[2]}(g)\right)=2 p_{x}^{1}(g) h_{p_{x}^{2} x}(g)-2 E
$$

and

$$
\begin{aligned}
p_{x}^{1} \mathrm{~d} D_{\left(g ; \omega^{[2]}(g)\right)} & h_{x x} \\
= & \mathrm{d}\left(p_{x}^{1} D_{\left(g ; \omega^{[2]}(g)\right)} h_{x x}\right)-\mathrm{d} p_{x}^{1} \wedge D_{\left(g ; \omega^{[2]}(g)\right)} h_{x x} \\
= & 2 \mathrm{~d}\left(p_{x}^{1}(g) h_{p_{x}^{2} x}(g)\right)-2 \mathrm{~d} E-\mathrm{d} p_{x}^{1} \wedge D_{\left(g ; \omega^{[2]}(g)\right)} h_{x x} \\
= & \mathrm{d} p_{x}^{1}(g) \wedge\left(2 h_{p_{x}^{2} x}(g)-D_{\left(g ; \omega^{[2]}(g)\right)} h_{x x}\right)+2 p_{x}^{1}(g) \mathrm{d} h_{p_{x}^{2} x}(g)-2 \mathrm{~d} E \\
= & 2 \mathrm{~d}\left(\hat{L}_{x}(g)\left(r_{-}{ }^{[1]}\right)\right) \wedge \hat{\Xi}\left(\operatorname{ad}_{r_{-}{ }^{[2]}} \hat{L}_{x}, \hat{L}_{x}\right)+2 p_{x}^{1}(g) \mathrm{d} h_{p_{x}^{2} x}(g)-2 \mathrm{~d} E \\
& =2 p_{x}^{1}(g) \mathrm{d} h_{p_{x}{ }^{2}}(g)-2 \hat{L}_{x}(g)\left(r_{-}{ }^{[1]}\right) \mathrm{d} \hat{\Xi}\left(\operatorname{ad}_{r_{-}{ }^{[2]}} \hat{L}_{x}, \hat{L}_{x}\right) .
\end{aligned}
$$


Using this with Lemma 4.13 and the ad-invariance of $\hat{\boldsymbol{E}}$ again,

$\left[x, \mathrm{~d} h_{x x}\right]_{\bullet}(v, w) / \hbar$

$=2 p_{x}^{1}(g) \mathrm{d} h_{p_{x}^{2} x}(g)(v, w)+\left(\hat{L}_{x} \otimes \mathrm{d} h_{x x}\right)(\hat{\Xi}(v) \otimes w-\hat{\Xi}(w) \otimes v)$

$+\hat{L}_{x}\left(r_{-}{ }^{[1]}\right) \mathrm{d}\left(\operatorname{ad}_{r_{-}[2]}^{*}\left(h_{x x}\right)-2 \hat{\Xi}\left(\operatorname{ad}_{r_{-}[2]}^{*} \hat{L}_{x}, \hat{L}_{x}\right)\right)(g)(v, w)+O(\hbar)$

$=2 p_{x}^{1}(g) \mathrm{d} h_{p_{x}^{2} x}(g)(v, w)+G(v, w)+O(\hbar)$,

where $G(v, w)=\left(\hat{L}_{x} \otimes \mathrm{d} h_{x x}\right)(\hat{\Xi}(v) \otimes w-\hat{\Xi}(w) \otimes v)$. This results in

$$
J_{2}(x, x, x)=3 G-2 \mathrm{~d} \hat{\Xi}\left(\hat{L}_{x}, h_{x x}\right) .
$$

Remember that $h_{x x}(g)(v)=x^{\prime}\left(g ; g \hat{\Xi}^{[1]}(v)\right) x^{\prime}\left(g ; g \hat{\Xi}^{[2]}(v)\right)$, so using Lemma 4.12, $\mathrm{d} h_{x x}(g)(w, v)=2 x^{\prime \prime}\left(g ; g \hat{\Xi}^{[1]}(v), g w\right) x^{\prime}\left(g ; g \hat{\Xi}^{[2]}(v)\right)$

$+2 x^{\prime}\left(g ; g w \hat{\Xi}^{[1]}(v)\right) x^{\prime}\left(g ; g \hat{\Xi}^{[2]}(v)\right)-2 x^{\prime \prime}\left(g ; g \hat{\Xi}^{[1]}(w), g v\right) x^{\prime}\left(g ; g \hat{\Xi}^{[2]}(w)\right)$

$-2 x^{\prime}\left(g ; g v \hat{\Xi}^{[1]}(w)\right) x^{\prime}\left(g ; g \hat{\Xi}^{[2]}(w)\right)-x^{\prime}\left(g ; g \hat{\Xi}^{[1]}([w, v])\right) x^{\prime}\left(g ; g \hat{\Xi}^{[2]}([w, v])\right)$.

Then, using the symmetry of $\hat{\Xi}$ and an independent copy $\hat{\Xi}^{\prime}$,

$$
\begin{aligned}
G(v, w)= & \hat{L}_{x}\left(\hat{\Xi}^{[1]}(v)\right) \mathrm{d} h_{x x}\left(\hat{\Xi}^{[2]}(v), w\right)-\hat{L}_{x}\left(\hat{\Xi}^{[1]}(w)\right) \mathrm{d} h_{x x}\left(\hat{\Xi}^{[2]}(w), v\right) \\
= & +2 x^{\prime \prime}\left(g ; g \hat{\Xi}^{\prime[1]}\left(\hat{\Xi}^{[2]}(w)\right), g v\right) \hat{L}_{x}\left(\hat{\Xi}^{\prime[2]}\left(\hat{\Xi}^{[2]}(w)\right)\right) \hat{L}_{x}\left(\hat{\Xi}^{[1]}(w)\right) \\
& -2 x^{\prime \prime}\left(g ; g \hat{\Xi}^{\prime[1]}\left(\hat{\Xi}^{[2]}(v)\right), g w\right) \hat{L}_{x}\left(\hat{\Xi}^{\prime[2]}\left(\hat{\Xi}^{[2]}(v)\right)\right) \hat{L}_{x}\left(\hat{\Xi}^{[1]}(v)\right) \\
& +2 \hat{L}_{x}\left(\hat{\Xi}^{[2]}(v) \hat{\Xi}^{\prime[1]}(w)\right) \hat{L}_{x}\left(\hat{\Xi}^{\prime[2]}(w)\right) \hat{L}_{x}\left(\hat{\Xi}^{[1]}(v)\right) \\
& -2 \hat{L}_{x}\left(w \hat{\Xi}^{\prime[1]}\left(\hat{\Xi}^{[2]}(v)\right)\right) \hat{L}_{x}\left(\hat{\Xi}^{\prime[2]}\left(\hat{\Xi}^{[2]}(v)\right)\right) \hat{L}_{x}\left(\hat{\Xi}^{[1]}(v)\right) \\
& -2 \hat{L}_{x}\left(\hat{\Xi}^{[2]}(w) \hat{\Xi}^{\prime[1]}(v)\right) \hat{L}_{x}\left(\hat{\Xi}^{\prime[2]}(v)\right) \hat{L}_{x}\left(\hat{\Xi}^{[1]}(w)\right) \\
& +2 \hat{L}_{x}\left(v \hat{\Xi}^{\prime[1]}\left(\hat{\Xi}^{[2]}(w)\right)\right) \hat{L}_{x}\left(\hat{\Xi}^{\prime[2]}\left(\hat{\Xi}^{[2]}(w)\right)\right) \hat{L}_{x}\left(\hat{\Xi}^{[1]}(w)\right) \\
& -\hat{L}_{x}\left(\hat{\Xi}^{\prime[1]}\left(\left[\hat{\Xi}^{[2]}(v), w\right]\right)\right) \hat{L}_{x}\left(\hat{\Xi}^{\prime[2]}\left(\left[\hat{\Xi}^{[2]}(v), w\right]\right)\right) \hat{L}_{x}\left(\hat{\Xi}^{[1]}(v)\right) \\
& +\hat{L}_{x}\left(\hat{\Xi}^{\prime[1]}\left(\left[\hat{\Xi}^{[2]}(w), v\right]\right)\right) \hat{L}_{x}\left(\hat{\Xi}^{\prime[2]}\left(\left[\hat{\Xi}^{[2]}(w), v\right]\right)\right) \hat{L}_{x}\left(\hat{\Xi}^{[1]}(w)\right) \\
= & +2 D_{(g ; g v)}\left(\hat{L}_{x}\left(\hat{\Xi}^{\prime[1]}\left(\hat{\Xi}^{[2]}(w)\right)\right)\right) \hat{L}_{x}\left(\hat{\Xi}^{\prime[2]}\left(\hat{\Xi}^{[2]}(w)\right)\right) \hat{L}_{x}\left(\hat{\Xi}^{[1]}(w)\right) \\
& -2 D_{(g ; g w)}\left(\hat{L}_{x}\left(\hat{\Xi}^{\prime[1]}\left(\hat{\Xi}^{[2]}(v)\right)\right)\right) \hat{L}_{x}\left(\hat{\Xi}^{\prime[2]}\left(\hat{\Xi}^{[2]}(v)\right)\right) \hat{L}_{x}\left(\hat{\Xi}^{[1]}(v)\right) \\
& +2 \hat{L}_{x}\left(\left[\hat{\Xi}^{[2]}(v), \hat{\Xi}^{\prime[1]}(w)\right]\right) \hat{L}_{x}\left(\hat{\Xi}^{\prime[2]}(w)\right) \hat{L}_{x}\left(\hat{\Xi}^{[1]}(v)\right) \\
& -\hat{L}_{x}\left(\hat{\Xi}^{\prime[1]}\left(\left[\hat{\Xi}^{[2]}(v), w\right]\right)\right) \hat{L}_{x}\left(\hat{\Xi}^{\prime[2]}\left(\left[\hat{\Xi}^{[2]}(v), w\right]\right)\right) \hat{L}_{x}\left(\hat{\Xi}^{[1]}(v)\right) \\
& +\hat{L}_{x}\left(\hat{\Xi}^{\prime[1]}\left(\left[\hat{\Xi}^{[2]}(w), v\right]\right)\right) \hat{L}_{x}\left(\hat{\Xi}^{\prime[2]}\left(\left[\hat{\Xi}^{[2]}(w), v\right]\right)\right) \hat{L}_{x}\left(\hat{\Xi}^{[1]}(w)\right) .
\end{aligned}
$$

Using Lemma 4.12, we obtain

$\mathrm{d} \hat{\boldsymbol{\Xi}}\left(\hat{L}_{x}, h_{x x}\right)(v, w)=D_{(g ; g v)}\left(\hat{L}_{x}\left(\hat{\boldsymbol{\Xi}}^{[1]}(w)\right) h_{x x}\left(\hat{\boldsymbol{\Xi}}^{[2]}(w)\right)\right)$

$$
-D_{(g ; g w)}\left(\hat{L}_{x}\left(\hat{\Xi}^{[1]}(v)\right) h_{x x}\left(\hat{\Xi}^{[2]}(v)\right)\right)-\hat{L}_{x}\left(\hat{\Xi}^{[1]}([v, w])\right) h_{x x}\left(\hat{\Xi}^{[2]}([v, w])\right),
$$


so

$$
\hat{L}_{x}\left(\hat{\Xi}^{[1]}(w)\right) h_{x x}\left(\hat{\Xi}^{[2]}(w)\right)=\hat{L}_{x}\left(\hat{\Xi}^{\prime[1]} \hat{\Xi}^{[2]}(w)\right) \hat{L}_{x}\left(\hat{\Xi}^{\prime[2]} \hat{\Xi}^{[2]}(w)\right) \hat{L}_{x}\left(\hat{\Xi}^{[1]}(w)\right)
$$

and

$$
\begin{aligned}
D_{(g ; g v)}\left(\hat{L}_{x}\left(\hat{\Xi}^{[1]}(w)\right) h_{x x}(\right. & \left.\left.\hat{\Xi}^{[2]}(w)\right)\right) \\
=2 D_{(g ; g v)}( & \left.\hat{L}_{x}\left(\hat{\Xi}^{\prime[1]} \hat{\Xi}^{[2]}(w)\right)\right) \hat{L}_{x}\left(\hat{\Xi}^{\prime[2]} \hat{\Xi}^{[2]}(w)\right) \hat{L}_{x}\left(\hat{\Xi}^{[1]}(w)\right) \\
& +\hat{L}_{x}\left(\hat{\Xi}^{\prime[1]} \hat{\Xi}^{[2]}(w)\right) \hat{L}_{x}\left(\hat{\Xi}^{\prime[2]} \hat{\Xi}^{[2]}(w)\right) D_{(g ; g v)}\left(\hat{L}_{x}\left(\hat{\Xi}^{[1]}(w)\right)\right) .
\end{aligned}
$$

Now we get

$$
\begin{aligned}
J_{2}(x, x, x)(v, w)= & +2 D_{(g ; g v)}\left(\hat{L}_{x}\left(\hat{\Xi}^{\prime[1]}\left(\hat{\Xi}^{[2]}(w)\right)\right)\right) \hat{L}_{x}\left(\hat{\Xi}^{\prime[2]}\left(\hat{\Xi}^{[2]}(w)\right)\right) \hat{L}_{x}\left(\hat{\Xi}^{[1]}(w)\right) \\
& -2 D_{(g ; g w)}\left(\hat{L}_{x}\left(\hat{\Xi}^{\prime[1]}\left(\hat{\Xi}^{[2]}(v)\right)\right)\right) \hat{L}_{x}\left(\hat{\Xi}^{\prime[2]}\left(\hat{\Xi}^{[2]}(v)\right)\right) \hat{L}_{x}\left(\hat{\Xi}^{[1]}(v)\right) \\
& +6 \hat{L}_{x}\left(\left[\hat{\Xi}^{[2]}(v), \hat{\Xi}^{\prime[1]}(w)\right]\right) \hat{L}_{x}\left(\hat{\Xi}^{\prime[2]}(w)\right) \hat{L}_{x}\left(\hat{\Xi}^{[1]}(v)\right) \\
& -3 \hat{L}_{x}\left(\hat{\Xi}^{\prime[1]}\left(\left[\hat{\Xi}^{[2]}(v), w\right]\right)\right) \hat{L}_{x}\left(\hat{\Xi}^{[2]}\left(\left[\hat{\Xi}^{[2]}(v), w\right]\right)\right) \hat{L}_{x}\left(\hat{\Xi}^{[1]}(v)\right) \\
& +3 \hat{L}_{x}\left(\hat{\Xi}^{\prime[1]}\left(\left[\hat{\Xi}^{[2]}(w), v\right]\right)\right) \hat{L}_{x}\left(\hat{\Xi}^{\prime[2]}\left(\left[\hat{\Xi}^{[2]}(w), v\right]\right)\right) \hat{L}_{x}\left(\hat{\Xi}^{[1]}(w)\right) \\
& -2 \hat{L}_{x}\left(\hat{\Xi}^{\prime[1]} \hat{\Xi}^{[2]}(w)\right) \hat{L}_{x}\left(\hat{\Xi}^{\prime[2]} \hat{\Xi}^{[2]}(w)\right) D_{(g ; g v)}\left(\hat{L}_{x}\left(\hat{\Xi}^{[1]}(w)\right)\right) \\
& +2 \hat{L}_{x}\left(\hat{\Xi}^{\prime[1]} \hat{\Xi}^{[2]}(v)\right) \hat{L}_{x}\left(\hat{\Xi}^{\prime[2]} \hat{\Xi}^{[2]}(v)\right) D_{(g ; g w)}\left(\hat{L}_{x}\left(\hat{\Xi}^{[1]}(v)\right)\right) \\
& +2 \hat{L}_{x}\left(\hat{\Xi}^{[1]}([v, w])\right) h_{x x}\left(\hat{\Xi}^{[2]}([v, w])\right) .
\end{aligned}
$$

By the ad-invariance of $\hat{\boldsymbol{\Xi}}^{\prime}$, we have

$\hat{\Xi}^{\prime}\left(\left[w, \hat{\Xi}^{[2]}(v)\right]\right)=\left[\hat{\Xi}^{\prime[1]}(w), \hat{\Xi}^{[2]}(v)\right] \otimes \hat{\Xi}^{\prime[2]}(w)+\hat{\Xi}^{\prime[1]}(w) \otimes\left[\hat{\Xi}^{\prime[2]}(w), \hat{\Xi}^{[2]}(v)\right]$ and

$$
\begin{aligned}
& \hat{L}_{x}\left(\hat{\Xi}^{[1]}([v, w])\right) h_{x x}\left(\hat{\Xi}^{[2]}([v, w])\right) \\
& =\hat{L}_{x}\left(\left[\hat{\Xi}^{[1]}(v), w\right]\right) \hat{L}_{x}\left(\hat{\Xi}^{\prime[1]} \hat{\Xi}^{[2]}(v)\right) \hat{L}_{x}\left(\hat{\Xi}^{\prime[2]} \hat{\Xi}^{[2]}(v)\right) \\
& \quad+2 \hat{L}_{x}\left(\hat{\Xi}^{[1]}(v)\right) \hat{L}_{x}\left(\left[\hat{\Xi}^{[2]}(v), \hat{\Xi}^{\prime[1]}(w)\right]\right) \hat{L}_{x}\left(\hat{\Xi}^{[2]}(w)\right),
\end{aligned}
$$

so we rewrite

$$
\begin{aligned}
J_{2}(x, x, x)(v, w)= & 2 D_{(g ; g v)}\left(\hat{L}_{x}\left(\hat{\Xi}^{[1]}\left(\hat{\Xi}^{[2]}(w)\right)\right)\right) \hat{L}_{x}\left(\hat{\Xi}^{\prime[2]}\left(\hat{\Xi}^{[2]}(w)\right)\right) \hat{L}_{x}\left(\hat{\Xi}^{[1]}(w)\right) \\
& -2 D_{(g ; g w)}\left(\hat{L}_{x}\left(\hat{\Xi}^{\prime[1]}\left(\hat{\Xi}^{[2]}(v)\right)\right)\right) \hat{L}_{x}\left(\hat{\Xi}^{\prime[2]}\left(\hat{\Xi}^{[2]}(v)\right)\right) \hat{L}_{x}\left(\hat{\Xi}^{[1]}(v)\right) \\
& -2 \hat{L}_{x}\left(\left[\hat{\Xi}^{[2]}(v), \hat{\Xi}^{\prime[1]}(w)\right]\right) \hat{L}_{x}\left(\hat{\Xi}^{\prime[2]}(w)\right) \hat{L}_{x}\left(\hat{\Xi}^{[1]}(v)\right) \\
& -2 \hat{L}_{x}\left(\hat{\Xi}^{\prime[1]} \hat{\Xi}^{[2]}(w)\right) \hat{L}_{x}\left(\hat{\Xi}^{\prime[2]} \hat{\Xi}^{[2]}(w)\right) D_{(g ; g v)}\left(\hat{L}_{x}\left(\hat{\Xi}^{[1]}(w)\right)\right) \\
& +2 \hat{L}_{x}\left(\hat{\Xi}^{\prime[1]} \hat{\Xi}^{[2]}(v)\right) \hat{L}_{x}\left(\hat{\Xi}^{\prime[2]} \hat{\Xi}^{[2]}(v)\right) D_{(g ; g w)}\left(\hat{L}_{x}\left(\hat{\Xi}^{[1]}(v)\right)\right) \\
& +\hat{L}_{x}\left(\left[\hat{\Xi}^{[1]}(v), w\right]\right) \hat{L}_{x}\left(\hat{\Xi}^{\prime[1]} \hat{\Xi}^{[2]}(v)\right) \hat{L}_{x}\left(\hat{\Xi}^{\prime[2]} \hat{\Xi}^{[2]}(v)\right) \\
& -\hat{L}_{x}\left(\left[\hat{\Xi}^{[1]}(w), v\right]\right) \hat{L}_{x}\left(\hat{\Xi}^{\prime[1]} \hat{\Xi}^{[2]}(w)\right) \hat{L}_{x}\left(\hat{\Xi}^{\prime[2]} \hat{\Xi}^{[2]}(w)\right) .
\end{aligned}
$$




\section{Setting}

$$
e(v)=\hat{\Xi}^{[1]}(v) \otimes \hat{\Xi}^{\prime[1]} \hat{\Xi}^{[2]}(v) \otimes \hat{\Xi}^{\prime[2]} \hat{\Xi}^{[2]}(v)-\hat{\Xi}^{\prime[1]} \hat{\Xi}^{[2]}(v) \otimes \hat{\Xi}^{[1]}(v) \otimes \hat{\Xi}^{\prime[2]} \hat{\Xi}^{[2]}(v),
$$

this becomes

$$
\begin{aligned}
J_{2}(x, x, x)(v, w)= & +2 D_{(g ; g w)}\left(\hat{L}_{x}\left(e^{[1]}(v)\right)\right) L_{x}\left(e^{[2]}(v)\right) L_{x}\left(e^{[3]}(v)\right) \\
& -2 D_{(g ; g v)}\left(\hat{L}_{x}\left(e^{[1]}(w)\right)\right) L_{x}\left(e^{[2]}(w)\right) L_{x}\left(e^{[3]}(w)\right) \\
& -2 \hat{L}_{x}\left(\left[\hat{\Xi}^{[2]}(v), \hat{\Xi}^{\prime[1]}(w)\right]\right) \hat{L}_{x}\left(\hat{\Xi}^{\prime[2]}(w)\right) \hat{L}_{x}\left(\hat{\Xi}^{[1]}(v)\right) \\
& +\hat{L}_{x}\left(\left[e^{[1]}(v), w\right]\right) \hat{L}_{x}\left(e^{[2]}(v)\right) \hat{L}_{x}\left(e^{[3]}(v)\right) \\
& -\hat{L}_{x}\left(\left[e^{[1]}(w), v\right]\right) \hat{L}_{x}\left(e^{[2]}(w)\right) \hat{L}_{x}\left(e^{[3]}(w)\right) \\
& +\hat{L}_{x}\left(\left[\hat{\Xi}^{\prime[1]} \hat{\Xi}^{[2]}(v), w\right]\right) \hat{L}_{x}\left(\hat{\Xi}^{[1]}(v)\right) \hat{L}_{x}\left(\hat{\Xi}^{\prime[2]} \hat{\Xi}^{[2]}(v)\right) \\
& -\hat{L}_{x}\left(\left[\hat{\Xi}^{\prime[1]} \hat{\Xi}^{[2]}(w), v\right]\right) \hat{L}_{x}\left(\hat{\Xi}^{[1]}(w)\right) \hat{L}_{x}\left(\hat{\Xi}^{\prime[2]} \hat{\Xi}^{[2]}(w)\right) .
\end{aligned}
$$

Now, using symmetry and ad-invariance,

$$
\begin{aligned}
& 2 \hat{L}_{x}\left(\left[\hat{\Xi}^{\prime[1]} \hat{\Xi}^{[2]}(v), w\right]\right) \hat{L}_{x}\left(\hat{\Xi}^{\prime[2]} \hat{\Xi}^{[2]}(v)\right) \\
& \quad=\hat{L}_{x}\left(\left[\hat{\Xi}^{\prime[1]} \hat{\Xi}^{[2]}(v), w\right]\right) \hat{L}_{x}\left(\hat{\Xi}^{\prime[2]} \hat{\Xi}^{[2]}(v)\right)+\hat{L}_{x}\left(\hat{\Xi}^{\prime[1]} \hat{\Xi}^{[2]}(v)\right) \hat{L}_{x}\left(\left[\hat{\Xi}^{\prime[2]} \hat{\Xi}^{[2]}(v), w\right]\right) \\
& \quad=\hat{L}_{x}\left(p^{[1]}\right) \hat{L}_{x}\left(p^{[2]}\right),
\end{aligned}
$$

where

$$
\begin{aligned}
p^{[1]} \otimes p^{[2]} & =\hat{\Xi}^{\prime}\left(\left[\hat{\Xi}^{[2]}(v), w\right]\right) \\
& =\left[\hat{\Xi}^{[2]}(v), \hat{\Xi}^{\prime[1]}(w)\right] \otimes \hat{\Xi}^{\prime[2]}(w)+\hat{\Xi}^{\prime[1]}(w) \otimes\left[\hat{\Xi}^{[2]}(v), \hat{\Xi}^{\prime[2]}(w)\right] .
\end{aligned}
$$

It follows that

$$
\hat{L}_{x}\left(\left[\hat{\Xi}^{\prime[1]} \hat{\Xi}^{[2]}(v), w\right]\right) \hat{L}_{x}\left(\hat{\Xi}^{\prime[2]} \hat{\Xi}^{[2]}(v)\right)=\hat{L}_{x}\left(\left[\hat{\Xi}^{[2]}(v), \hat{\Xi}^{\prime[1]}(w)\right]\right) \hat{L}_{x}\left(\hat{\Xi}^{\prime[2]}(w)\right),
$$

and so

$$
\begin{aligned}
J_{2}(x, x, x)(v, w)= & +2 D_{(g ; g w)}\left(\hat{L}_{x}\left(e^{[1]}(v)\right)\right) \hat{L}_{x}\left(e^{[2]}(v)\right) \hat{L}_{x}\left(e^{[3]}(v)\right) \\
& -2 D_{(g ; g v)}\left(\hat{L}_{x}\left(e^{[1]}(w)\right)\right) \hat{L}_{x}\left(e^{[2]}(w)\right) \hat{L}_{x}\left(e^{[3]}(w)\right) \\
& +\hat{L}_{x}\left(\left[e^{[1]}(v), w\right]\right) \hat{L}_{x}\left(e^{[2]}(v)\right) \hat{L}_{x}\left(e^{[3]}(v)\right) \\
& -\hat{L}_{x}\left(\left[e^{[1]}(w), v\right]\right) \hat{L}_{x}\left(e^{[2]}(w)\right) \hat{L}_{x}\left(e^{[3]}(w)\right) .
\end{aligned}
$$

Use of the first super-Jacobi result yields $e(v)=-n^{[1]} \otimes n^{[2]} \otimes\left[v, n^{[3]}\right]$, as stated.

From Section 2 we know that if $J_{1}, J_{2}$ vanish then the third super-Jacobi identity also holds. Putting together several of the results above we find as a special case: 
Theorem 4.18. Every quastriangular Poisson-Lie group has a compatible bicovariant preconnection given by $\hat{\Xi}=0$. The corresponding $\tilde{\gamma}$ is given by

$$
\tilde{\gamma}(a, s)(g)=\{a, s\}(g)+a^{\prime}\left(g ; g r_{-}{ }^{[1]}\right) \operatorname{ad}_{r_{-}[2]}^{*}(s(g))
$$

for $a \in C^{\infty}(G)$ and $s \in C^{\infty}\left(G, \mathfrak{g}^{*}\right)$. If $\mathfrak{g}$ is semisimple then the curvature vanishes if and only if the Lie bialgebra is triangular, and in this case all super-Jacobi identities $J_{i}=0$ hold.

Proof. Clearly $\hat{\boldsymbol{E}}=0$ is symmetric and ad*-invariant, hence by Corollary 4.7 and Proposition 4.15 defines a bicovariant preconnection. To work out what it looks like we have only to work backwards from the definitions of $\hat{\Xi}, \Xi$ and Definition 4.4 to find the result stated. If $\hat{\boldsymbol{\Xi}} \neq 0$ we would need an additional term $\hat{\boldsymbol{\Xi}}\left(\hat{L}_{x}(g), s(g)\right)$. For the second part, the Lie bialgebra is triangular precisely when $n=\llbracket r_{-}, r_{-} \rrbracket=$ $\left[r_{+12}, r_{+23}\right]$ vanishes, which is if and only if $r_{+}=0$ since this is either zero or nondegenerate in the semisimple case. Also in this case $e(v)=0$ for all $v$ if and only if $n=0$, which is the case for the curvature obstruction to $J_{1}$ to vanish according to Proposition 4.16 and the interpretation in Section 2. In this case $J_{2}$ also vanishes by the preceding proposition and hence $J_{3}$ by Proposition 2.6.

Proposition 4.19. The above canonical choice of compatible preconnection $\gamma$ on a quasitriangular Poisson-Lie group is given explicitly by

$$
\gamma(a, \tau)=\left(a \triangleleft r_{-}{ }^{[1]}\right)\left(\tau \triangleleft r_{-}{ }^{[2]}\right)-\left(r_{-}{ }^{[1]} \triangleright a\right)\left(r_{-}{ }^{[2]} \triangleright \tau\right)
$$

where $\triangleleft$ is the right action on functions or 1-forms corresponding to $\Delta, \Delta_{R}$ respectively. Its curvature and torsion are

$$
\begin{aligned}
R(x, y) \tau & =\left(n^{[1]} \triangleright x\right)\left(n^{[2]} \triangleright y\right)\left(n^{[3]} \triangleright \tau\right)-\left(x \triangleleft n^{[1]}\right)\left(y \triangleleft n^{[2]}\right)\left(\tau \triangleleft n^{[3]}\right), \\
T(x, y)(\mathrm{d} z) & =\left(m^{[1]} \triangleright x\right)\left(m^{[2]} \triangleright y\right)\left(m^{[3]} \triangleright z\right)-\left(x \triangleleft m^{[1]}\right)\left(y \triangleleft m^{[2]}\right)\left(z \triangleleft m^{[3]}\right),
\end{aligned}
$$

where $n=\llbracket r_{-}, r_{-} \rrbracket$ and $m=\left[r_{-13}, r_{-23}\right]$.

Proof. Here the right actions are defined in the same way as we did for $\triangleright$ at the start of Section 4A, so $(a \triangleleft g)(h)=a\left(h g^{-1}\right)$ on functions and $(\tau \triangleleft g)(h)=$ $R_{g^{-1}}^{*}\left(\tau\left(h g^{-1}\right)\right)$ on forms, and we use the infinitesimal versions. Unwinding our definition of $\tilde{\gamma}$, we have

$$
\begin{aligned}
\gamma(a, & \tau)(g) \\
& =L_{g^{-1}}^{*}(\tilde{\gamma}(a, \tilde{\tau})(g)) \\
& \left.=L_{g^{-1}}^{*}\left(a^{\prime}\left(g ; g r_{-}{ }^{[1]}\right)\left(\tilde{\tau}^{\prime}\left(g ; g r_{-}{ }^{[2]}\right)+\operatorname{ad}_{r_{-}}^{*}(\tilde{c}](g)\right)\right)-a^{\prime}\left(g ; r_{-}{ }^{[1]} g\right) \tilde{\tau}^{\prime}\left(g ; r_{-}{ }^{[2]} g\right)\right) \\
& =a^{\prime}\left(g ; r_{-}{ }^{[1]} g\right)\left(r_{-}{ }^{[2]} \triangleright \tau\right)(g)-a^{\prime}\left(g ; g r_{-}{ }^{[1]}\right)\left(\tau \triangleleft r_{-}{ }^{[2]}\right)(g),
\end{aligned}
$$

as stated. Here 


$$
a^{\prime}(g ; g v)=\left(L_{* v} a\right)(g)=-(a \triangleleft v)(g), \quad a^{\prime}(g ; v g)=\left(R_{* v} a\right)(g)=-(v \triangleright a)(g)
$$

for $v \in \mathfrak{g}$. On forms, we similarly have

$$
\begin{aligned}
L_{g^{-1}}^{*}\left(\tilde{\tau}^{\prime}(g ; v g)\right) & =\left.L_{g^{-1}}^{*} \frac{\mathrm{d}}{\mathrm{d} t}\right|_{0} \tilde{\tau}\left(e^{t v} g\right)=\left.\frac{\mathrm{d}}{\mathrm{d} t}\right|_{0} L_{e^{t v}}^{*}\left(\tau\left(e^{t v} g\right)\right) \\
& =\left.\frac{\mathrm{d}}{\mathrm{d} t}\right|_{0}\left(e^{-t v} \triangleright \tau\right)(g)=-(v \triangleright \tau)(g), \\
L_{g^{-1}}^{*}\left(\tilde{\tau}^{\prime}(g ; g v)+\operatorname{ad}_{v}^{*}(\tilde{\tau}(g))\right) & =\left.L_{g^{-1}}^{*} \frac{\mathrm{d}}{\mathrm{d} t}\right|_{0} \operatorname{Ad}_{e^{t v}}^{*}\left(\tilde{\tau}\left(g e^{t v}\right)\right)=\left.\frac{\mathrm{d}}{\mathrm{d} t}\right|_{0} R_{e^{t v}}^{*}\left(\tau\left(g e^{t v}\right)\right) \\
& =\left.\frac{\mathrm{d}}{\mathrm{d} t}\right|_{0}\left(\tau \triangleleft e^{-t v}\right)(g)=-(\tau \triangleleft v)(g) .
\end{aligned}
$$

This is equivalent to the computation in Lemma 4.10 for exact forms. Next, it is a useful check to compute the curvature directly form the definition (3-1), with result in line with Proposition 4.16. We use the canonical $\gamma$ and its connection property (2-4) to write

$$
\begin{aligned}
R(x, y) \tau= & \gamma(x, \gamma(y, \tau))-\gamma(y, \gamma(x, \tau))-\gamma(\{x, y\}, \tau) \\
= & \gamma\left(x,\left(y \triangleleft r_{-}{ }^{[1]}\right)\left(\tau \triangleleft r_{-}{ }^{[2]}\right)-\left(r_{-}{ }^{[1]} \triangleright y\right)\left(r_{-}{ }^{[2]} \triangleright \tau\right)\right) \\
& \quad-(x \leftrightarrow y)-\left(\{x, y\} \triangleleft r_{-}{ }^{[1]}\right)\left(\tau \triangleleft r_{-}{ }^{[2]}\right)+\left(r_{-}{ }^{[1]} \triangleright\{x, y\}\right)\left(r_{-}{ }^{[2]} \triangleright \tau\right) .
\end{aligned}
$$

We then insert the formulae for $\gamma$ and $\{$,$\} (as discussed below) and expand \triangleright, \triangleleft$ as derivations to obtain 24 terms. Cancelling 12 and using that $\triangleright, \triangleleft$ are mutually commuting actions of $\mathfrak{g}$, together with the antisymmetry of $r_{-}$, we obtain the result. Finally, using the expression for torsion as in Proposition 3.2 we have

$$
\begin{aligned}
\langle\mathrm{d} z, T(x, y)\rangle & =\langle\hat{x}, \gamma(y, \mathrm{~d} z)\rangle-\langle\hat{y}, \gamma(x, \mathrm{~d} z)\rangle \\
& =\left\langle\hat{x},\left(y \triangleleft r_{-}{ }^{[1]}\right) \mathrm{d}\left(z \triangleleft r_{-}{ }^{[2]}\right)-\left(r_{-}{ }^{[1]} \triangleright y\right) \mathrm{d}\left(r_{-}{ }^{[2]} \triangleright z\right)\right\rangle-(x \leftrightarrow y) \\
& =\left(y \triangleleft r_{-}{ }^{[1]}\right)\left\{x, z \triangleleft r_{-}{ }^{[2]}\right\}-\left(r_{-}{ }^{[1]} \triangleright y\right)\left\{x, r_{-}{ }^{[2]} \triangleright z\right\}-(x \leftrightarrow y),
\end{aligned}
$$

where we used $\gamma$ on exact forms as discussed below. We then expand out the Poisson bracket and cancel terms. One may similarly compute $T(x, y)(\tau)$ in general.

Since d commutes with the actions, or by Lemma 4.10, we have in particular

$$
\gamma(a, \mathrm{~d} b)=\left(a \triangleleft r_{-}{ }^{[1]}\right) \mathrm{d}\left(b \triangleleft r_{-}{ }^{[2]}\right)-\left(r_{-}{ }^{[1]} \triangleright a\right) \mathrm{d}\left(r_{-}{ }^{[2]} \triangleright b\right),
$$

while, in the same notation,

$$
\{a, b\}=\left(a \triangleleft r_{-}{ }^{[1]}\right)\left(b \triangleleft r_{-}{ }^{[2]}\right)-\left(r_{-}{ }^{[1]} \triangleright a\right)\left(r_{-}{ }^{[2]} \triangleright b\right)
$$

coincides with the usual formula on a quasitriangular Poisson-Lie group (because one could equally well put $r$ here since $r_{+}$is ad-invariant). Then the antisymmetry 
of $r_{-}$and the Leibniz rule confirm that the canonical connection is compatible in the sense of (2-5). Also, the torsion is consistent with Proposition 3.2 since on cyclic summation, $\left[r_{-13}, r_{-23}\right]$ becomes replaced by $\llbracket r_{-}, r_{-} \rrbracket$ and this now gives zero as the statement that (4-8) obeys the Jacobi identity (usually this is done via $\llbracket r, r \rrbracket=0)$. By contrast, the action of $\llbracket r_{-}, r_{-} \rrbracket$ in the curvature, even on exact forms, is not trivial. We note also that the same formula as for the canonical connection but with $r$ in place of $r_{-}$is what one obtains by semiclassicalising the 'quantum Lie functor' construction in [Gomez and Majid 2003] for a canonical bicovariant quantum differential calculus on any coquasitriangular Hopf algebra. There the coquasitriangular structure $\mathscr{R}$ or 'universal R-matrix' plays the role of $r$. Unfortunately, that functor only gives nontrivial answers in the triangular case and now we can see why: only in this case is $r=r_{-}$, so that it coincides with our result above. Moreover, only this case corresponds to $J_{1}=0$ and hence to a bimodule on quantization.

Finally, we use Corollary 4.7 and Proposition 4.17 to study the entire moduli space of bicovariant semiclassical calculi. These results tell us that the moduli space is an affine space with the above canonical preconnection as reference and others given by the vector space of Ad-invariant symmetric maps $\hat{\Xi}: \mathfrak{g} \rightarrow \operatorname{Sym}^{2}(\mathfrak{g})$. Such maps may be classified for $\mathfrak{g}$ reductive using Kostant's results on harmonic functions in [Kostant 1963]. In particular:

Theorem 4.20. If $\mathfrak{g}$ is simple and not $\mathfrak{s l}_{n}, n>2$, the moduli space is a point, i.e., the canonical preconnection $\hat{\boldsymbol{\Xi}}=0$ is the unique bicovariant one. If $\mathfrak{g}=\mathfrak{s l}_{n}, n>2$, the moduli space is a 1-parameter family given by $\hat{\boldsymbol{\Xi}}$ a multiple of the split cubic Casimir in $\mathfrak{g} \otimes \mathfrak{g} \otimes \mathfrak{g}$.

Proof. Since $\mathfrak{g}$ is simple $\hat{\Xi}$ is zero or an inclusion, so we need the multiplicity of $\mathfrak{g}$ in the symmetric tensor square $\operatorname{Sym}^{2}(\mathfrak{g})$. We freely use the Ad-invariant Killing form to identify $\mathfrak{g}$ and $\mathfrak{g}^{*}$ for our purposes, so this is equivalent to the multiplicity of $\mathfrak{g}$ in the symmetric polynomials $S^{2}(\mathfrak{g})$ of degree 2 on $\mathfrak{g}$. From [Kostant 1963], $S(\mathfrak{g})=J \otimes$ Harm as a vector space, where $J$ denotes the invariant polynomials and Harm the harmonic ones. Hence $S^{2}(\mathfrak{g})=J^{2} \oplus\left(J^{1} \otimes \mathrm{Harm}^{1}\right) \oplus \mathrm{Harm}^{2} . J$ is well-known to be generated by functions $\left\{u_{i}\right\}$ of degrees $m_{i}+1$ where $m_{i}$ are the 'exponents' of the Lie algebra. Clearly $J^{2}$ is 1-dimensional (spanned by the Killing form) and $J^{1}=0$, so we need only to classify embeddings of $\mathfrak{g}$ in $\mathrm{Harm}^{2}$. From [Kostant 1963, Theorem 0.11] the component of Harm transforming as a given highest weight representation $\lambda$ consists of $l_{\lambda}$ copies with certain degrees $m_{i}(\lambda)$. In the case of the adjoint representation these reduce to the usual rank $l$ and exponents $m_{i}$. Finally, we turn to tables and find that only $\mathfrak{s l}_{n}, n>2$ has an exponent with value 2 (a cubic Casimir). Other simple Lie algebras have nothing in Harm of degree 2 and hence have $\hat{\boldsymbol{E}}=0$. In the case of $\mathfrak{s l}_{n}, n>2$ there is one exponent 
with value 2 (namely $m_{2}$ ) so there is a single copy of $\mathfrak{g}$ in the decomposition of $S^{2}(\mathfrak{g})$. It is necessarily given by the corresponding unique totally symmetric invariant element $u_{2} \in J^{3}$, viewed as a map $\mathfrak{g} \rightarrow S^{2}(\mathfrak{g})$. From another point of view this corresponds to the cubic Casimir in the enveloping algebra, or the unique totally symmetric 'cubic split Casimir' in $\operatorname{Sym}^{3}(\mathfrak{g})$. Thus up to normalisation, $\hat{\boldsymbol{E}}$ is necessarily this element viewed as $\mathfrak{g}^{*} \rightarrow \operatorname{Sym}^{2}(\mathfrak{g})$ by evaluation in one input and converted to $\mathfrak{g} \rightarrow \operatorname{Sym}^{2}(\mathfrak{g})$ with the help of the Killing form.

We see that for all but the $\mathfrak{s l}_{n}$ series, the corresponding Poisson-Lie group with any fixed strictly quasitriangular structure (such as the standard one) admits a unique semiclassical bicovariant calculus, and its preconnection has nonzero curvature. Therefore it cannot be quantised even at a bimodule level; that is, there can be no first order bicovariant different calculus on the standard quantum groups of the same dimension as the classical one. This agrees with what is known from quantum group theory by other means [Majid 1998a]. The 1-parameter family for $\mathfrak{s l}_{n}, n>2$ at least generically also has curvature from Proposition 4.17, and hence the same problem.

4E. Example: canonical connection on $\mathrm{SU}_{2}$. We take the same Poisson-Lie group as in Section $4 \mathrm{C}$ and the notations there. This time there is no known quantum calculus to semiclassicalise but rather we use our results above. In this case one can see directly that $\mathfrak{s l}_{2}$ is not to be found in the symmetric part of $\mathfrak{s l}_{2} \otimes \mathfrak{s l}_{2}$ (which is the $1 \oplus 5$ dimensional representation under ad), hence the only bicovariant preconnection by Proposition 4.15 is $\hat{\Xi}=0$, as per Theorem 4.20.

In this case Theorem 4.18 says that $\tilde{\gamma}\left(x, \tilde{\tau}^{i}\right)=\left(L_{* r_{-}[1]} x\right) \operatorname{ad}_{r_{-}[2]}^{*} \tilde{\tau}^{i}$ since the $\tilde{\tau}^{i}$ are constant. Also for this reason, we have the same formula without all the tildes, with the $\tau^{i}$ transforming in the same way under $\mathrm{ad}^{*}$ as the $\tilde{\tau}^{i}$. Putting in the form of $r_{-}$in our case, we have

$$
\gamma\left(x, \tau^{i}\right)=\frac{1}{2}\left(\left(\partial_{+} x\right) \operatorname{ad}_{e_{-}}^{*}\left(\tau^{i}\right)-\left(\partial_{-} x\right) \operatorname{ad}_{e_{+}}^{*}\left(\tau^{i}\right)\right) .
$$

Computing $\operatorname{ad}_{e_{i}}^{*}$ we find

$\gamma\left(x, \tau^{+}\right)=-\left(\partial_{-} x\right) \tau^{3}, \quad \gamma\left(x, \tau^{-}\right)=-\left(\partial_{+} x\right) \tau^{3}, \quad \gamma\left(x, \tau^{3}\right)=\frac{1}{2}\left(\left(\partial_{+} x\right) \tau^{+}+\left(\partial_{-} x\right) \tau^{-}\right)$.

As we expect from the Proposition 4.19, this compatible preconnection has both curvature and torsion. Hence by Sections 2 and 3 it cannot be quantized to an honest bimodule first order quantum calculus let alone higher forms.

\section{Quasiassociative exterior algebras}

Here we give a setting for the quantisation of Poisson-Lie groups where the preconnection has nonzero curvature, such as the canonical one in Theorem 4.18 for the 
strictly quasitriangular case. This case includes the standard Drinfeld-Sklyanin Poisson-Lie groups as demonstrated for $\mathrm{SU}_{2}$ in Section 4E. In such a case of curvature we know that the quantisation must be nonassociative. We show now that this can be controlled nicely in the setting of coquasi-Hopf algebras, where there is a Drinfeld associator $\Phi$. We first work out the relevant noncommutative differential geometry first and then semiclassicalise it. As a result, we succeed to quantise the canonical connection for such quasitriangular Poisson Lie groups.

For general constructions we work over a field $k$, which can also (with care) be replaced by commutative ring such as $\mathbb{C} \llbracket \hbar \rrbracket$ for the formal deformation theory. Our starting point is a class of natural examples obtained by twisting as follows. If $H$ is a Hopf algebra and $F: H \otimes H \rightarrow k$ is a 2-cochain in the sense of convolution invertible in the form $F\left(a_{(1)} \otimes b_{(1)}\right) F^{-1}\left(a_{(2)} \otimes b_{(2)}\right)=\epsilon(a) \epsilon(b)$ (and similarly on the other side) and obeying $F(1 \otimes a)=\epsilon(a)$ then one may define a new object $H_{F}$ with modified product

$$
a \bullet b=F\left(a_{(1)} \otimes b_{(1)}\right) a_{(2)} b_{(2)} F^{-1}\left(a_{(3)} \otimes b_{(3)}\right) \quad \text { for all } a, b \in H .
$$

This is not necessarily associative but rather we have

$$
a \bullet(b \bullet c)=\Phi\left(a_{(1)} \otimes b_{(1)} \otimes c_{(1)}\right)\left(a_{(2)} \bullet b_{(2)}\right) \bullet c_{(2)} \Phi^{-1}\left(a_{(3)} \otimes b_{(3)} \otimes c_{(3)}\right),
$$

where

$$
\Phi(a \otimes b \otimes c)=F\left(b_{(1)} \otimes c_{(1)}\right) F\left(a_{(1)} \otimes b_{(2)} c_{(2)}\right) F^{-1}\left(a_{(2)} b_{(3)} \otimes c_{(3)}\right) F^{-1}\left(a_{(3)} \otimes b_{(4)}\right)
$$

is the coboundary of $F$ in some kind of nonabelian cohomology [Majid 1995]. This makes $H_{F}$ into an example of a coquasi-Hopf algebra. By definition the latter is defined to be a coalgebra which has a product obeying (5-1) with respect to some 3 -cocycle $\Phi$ (not necessarily of the coboundary form). There should also be an antipode $S$ obeying certain axioms. The axioms in a nondual form are due to Drinfeld [1989], who showed what would in our setting be the following assertion: the standard coquasitriangular quantum groups $C_{\hbar}(G)$ are (nontrivially) isomorphic to twists of the classical functions $C(G)$ (in some form) by a formal cochain $F=F_{\hbar}$. Although the $F$ needed here is not a cocycle so that $\Phi$ is not trivial, $\Phi$ turns out in this example to be cocentral and hence disappears from (5-1) with the result that $C_{\hbar}(G)$ is an ordinary Hopf algebra. We do not need the fully precise formulation but only the idea and formulae to lowest order, which we will provide.

The second idea is the Majid-Oeckl theorem for twisting of bicovariant differential calculi on Hopf algebras. It is known [Brzeziński 1993] that in the bicovariant case the Woronowicz exterior algebra $\Omega(H)$ as a whole forms a super-Hopf algebra. It is just $H$ in degree 0 and in higher degree the coproduct is generated by

$$
\Delta=\Delta_{L}+\Delta_{R}
$$


in degree 1 . We will use the notation

$$
\left(\Delta_{L} \otimes \mathrm{id}\right) \Delta_{R} \tau=\left(\mathrm{id} \otimes \Delta_{R}\right) \Delta_{L} \tau=\tau_{(1)} \otimes \tau_{(2)} \otimes \tau_{(3)} \in H \otimes \Omega^{1} \otimes H
$$

for any 1 -form $\tau$. The theorem of [Majid and Oeckl 1999] states that if $F$ is a 2-cocycle (so that $H_{F}$ is again a Hopf algebra) then $H_{F}$ has a natural bicovariant differential calculus given by $\Omega\left(H_{F}\right)=\Omega(H)_{F}$, where we twist $\Omega(H)$ as a superHopf algebra with $F$ extended by zero on higher degrees. The twisted module and wedge product are

$$
\begin{aligned}
a \bullet \tau & =F\left(a_{(1)} \otimes \tau_{(1)}\right) a_{(2)} \tau_{(2)} F^{-1}\left(a_{(3)} \otimes \tau_{(3)}\right) \\
\tau \bullet a & =F\left(\tau_{(1)} \otimes a_{(1)}\right) \tau_{(2)} a_{(2)} F^{-1}\left(\tau_{(3)} \otimes a_{(3)}\right) \\
\tau \wedge \bullet \eta & =F\left(\tau_{(1)} \otimes \eta_{(1)}\right) \tau_{(2)} \wedge \eta_{(2)} F^{-1}\left(\eta_{(3)} \otimes \eta_{(3)}\right)
\end{aligned}
$$

for $a \in H$ and 1-forms $\tau, \eta$. Note that ( $\Delta \otimes$ id) $\Delta \tau$ contains three terms, but only the middle one (5-2) enters in the above expressions since $F$ pairs only in degree zero. The same applies in formulae below.

We can ask what kind of object does one get if $F$ is not a cocycle. According to the above, then $\Omega\left(H_{F}\right)$ given by twisting will now be a super coquasi-Hopf algebra. Looking at such an example we have the following definition and proposition:

Definition 5.1. If $(H, \Phi)$ is a coquasi-Hopf algebra, we define its first order quasi differential calculus $\Omega^{1}(H)$ to be $\left(\Omega^{1}\right.$, d) where $\Omega^{1}$ is a quasibimodule in the sense

$$
\begin{aligned}
& a \bullet(b \bullet \tau)=\Phi\left(a_{(1)} \otimes b_{(1)} \otimes \tau_{(1)}\right)\left(a_{(2)} \bullet b_{(2)}\right) \bullet \tau_{(2)} \Phi^{-1}\left(a_{(3)} \otimes b_{(3)} \otimes \tau_{(3)}\right) \\
& \tau \bullet(b \bullet c)=\Phi\left(\tau_{(1)} \otimes b_{(1)} \otimes c_{(1)}\right)\left(\tau_{(2)} \bullet b_{(2)}\right) \bullet c_{(2)} \Phi^{-1}\left(\tau_{(3)} \otimes b_{(3)} \otimes c_{(3)}\right) \\
& a \bullet(\tau \bullet c)=\Phi\left(a_{(1)} \otimes \tau_{(1)} \otimes c_{(1)}\right)\left(a_{(2)} \bullet \tau_{(2)}\right) \bullet c_{(2)} \Phi^{-1}\left(a_{(3)} \otimes \tau_{(3)} \otimes c_{(3)}\right)
\end{aligned}
$$

and the rest as usual (so $d$ is a derivation with respect to the product $\bullet$, etc and the calculus is bicovariant if coactions $\Delta_{L}, \Delta_{R}$ commute with $\bullet$ and intertwine d).

Proposition 5.2. If $H$ is an ordinary Hopf algebra and $F$ a 2-cochain then $\Omega^{1}\left(H_{F}\right)$ defined by (5-3) and the same $\mathrm{d}$ is a first order quasidifferential calculus on $H_{F}$.

Proof. This is more or less by construction. We define $\Omega\left(H_{F}\right)$ namely as a super coquasi-Hopf algebra given by $\Omega(H)_{F}$. As such it obeys the conditions in Definition 5.1 as these are just the lowest order part of the assertion that $\Omega\left(H_{F}\right)$ is a super coquasi-Hopf algebra. One may check that $d$ remains a derivation, the proof being the same as in [Majid and Oeckl 1999].

We do not go through all the steps of the Woronowicz construction for the exterior algebra $\Omega(H)$ in detail but this may surely be done and is straightforward in view of the category of quasicrossed modules being known [Majid 1998b]. Then the entire $\Omega\left(H_{F}\right)$ will be an example of such a general construction. 
In particular, by the remarks above, all the standard coquasitriangular quantum groups $C_{\hbar}(G)$ have such bicovariant quasidifferential calculi $\Omega^{1}\left(C_{\hbar}(G)\right)$ and indeed an entire super coquasi-Hopf exterior algebra. We now make a semiclassical analysis to lowest order of this example. What we need from Drinfeld's theory is that

$$
F_{\hbar}=1 \otimes 1+\hbar f+O\left(\hbar^{2}\right) \in U(\mathfrak{g}) \otimes U(\mathfrak{g}),
$$

where $\mathfrak{g}$ is the Lie algebra of $G$. Since it is given by twisting from its symmetric part, the quasitriangular structure is

$$
r=f_{21}-f+r_{+}
$$

where $r_{+}$is the split Casimir or inverse Killing form in a suitable normalisation. The antisymmetric part is $r_{-}=f_{21}-f$. Moreover,

$$
\Phi=1 \otimes 1 \otimes 1-\frac{1}{6} \hbar^{2} \llbracket r_{-}, r_{-} \rrbracket+O\left(\hbar^{3}\right) .
$$

The following applies to these standard Poisson-Lie groups and any others where the quasitriangular Poisson-Lie group can be quantized to a coquasitriangular Hopf algebra by a cochain twist, with the induced twisted quantum differential calculus.

Proposition 5.3. The preconnection obtained by semiclassicalizing the quantum differential calculi obtained by cochain-twisting is the canonical one $\hat{\Xi}=0$ in Proposition 4.19.

Proof. In the twisted bicovariant calculus we have

$$
\begin{aligned}
a \bullet \tau & =a \tau+\hbar\left(\left\langle f, a_{(1)} \otimes \tau_{(1)}\right\rangle a_{(2)} \tau_{(\infty)}-a_{(1)} \tau_{(0)}\left\langle f, a_{(2)} \otimes \tau_{(1)}\right\rangle\right)+O\left(\hbar^{2}\right) \\
\tau \bullet a & =\tau a+\hbar\left(\left\langle f, \tau_{(1)} \otimes a_{(1)}\right\rangle \tau_{(\infty)} a_{(2)}-\tau_{(0)} a_{(1)}\left\langle f, \tau_{(1)} \otimes a_{(2)}\right\rangle\right)+O\left(\hbar^{2}\right) \\
{[a, \tau]_{\bullet} } & =\hbar\left(\left\langle f-f_{21}, a_{(1)} \otimes \tau_{(1)}\right\rangle a_{(2)} \tau_{(\infty)}-a_{(1)} \tau_{(0)}\left\langle f-f_{21}, a_{(2)} \otimes \tau_{(1)}\right\rangle\right)+O\left(\hbar^{2}\right) \\
& =\hbar\left(\left(a \triangleleft r_{-}{ }^{[1]}\right)\left(\tau \triangleleft r_{-}{ }^{[2]}\right)-\left(r_{-}{ }^{[1]} \triangleright a\right)\left(r_{-}{ }^{[2]} \triangleright \tau\right)\right)+O\left(\hbar^{2}\right),
\end{aligned}
$$

as follows. First, when we expand $F, F^{-1}$, the $1 \otimes 1$ of one or other of these evaluates against $(\Delta \otimes \mathrm{id}) \Delta \tau$ to yield $\Delta \tau=\Delta_{L} \tau+\Delta_{R} \tau$ and of these two terms only will contribute as $F$ pairs only in degree zero. We recognize $-r_{-}$appearing here. Finally, we note that if $v \in \mathfrak{g}$ then

$$
\left\langle v, a_{(1)}\right\rangle a_{(2)}=-v \triangleright a=R_{* v}(a)
$$

and $\left\langle v, \tau_{(1)}\right\rangle \tau_{(\infty)}=-v \triangleright \tau$ in the conventions of Section 4A, and similarly on the other side. Comparing, we have exactly the formula in Proposition 4.19, which corresponds to $\hat{\Xi}=0$.

In view of Proposition 4.19 we also see the nonassociativity of the exterior algebra reflected in the curvature of the associated preconnection. Also, there is nothing stopping one doing the above with a cochain where $\Phi$ is not central in the 
sense above. Then one obtains a quasidifferential calculus on a quasi-Hopf algebra $H_{F}$. In this case the infinitesimal data even for the function algebra is not a Poisson bracket but something weaker as in [Kosmann-Schwarzbach 1992].

\section{The Fedosov point of view}

The reader may wonder where the Fedosov [1996] method of deformation quantisation fits into this picture of deforming differential calculus that we have developed. Since the initial data in [Fedosov 1996] is a symplectic structure and a symplectic connection, it seems that its correct formulation could be as not only quantising functions but functions and differentials. We first point out that there are indeed flat sections of the bundle of Weyl differential forms, and the wedge product of such sections is flat. All multiplications are associative, and the forms are a left and right module over the functions on the manifold. The reader is reminded that the functions on the manifold are replaced in the Fedosov theory by the flat sections of the 0-form Weyl bundle, $W_{D}$ in the notation of [Fedosov 1996]. Since these flat sections are in a natural 1-1 correspondence with the ordinary functions on the manifold, it seems that we have deformed the product on $C^{\infty}(M)$ itself. The problem with the $q$-forms for $q \geq 1$ is that there are far too many flat sections of the $q$-form Weyl bundle, as we now see:

Proposition 6.1. For $q \geq 1$ there is a 1-1 correspondence

$$
\delta \delta^{-1}:\left\{\tau \in C^{\infty}\left(W \otimes \Lambda^{q}\right): D \tau=0\right\} \longrightarrow\left\{\eta \in C^{\infty}\left(W \otimes \Lambda^{q}\right): \delta \eta=0\right\} .
$$

The inverse map sends $\eta$ to $\tau$, the unique solution of the equation

$$
\tau=\delta^{-1}(D+\delta) \tau+\eta
$$

Proof. That (6-1) has a solution can be seen by an iterative method. Set $\tau_{0}=\eta$, and continue with

$$
\tau_{n+1}=\delta^{-1}(D+\delta) \tau_{n}+\eta
$$

Then we have

$$
\tau_{n+2}-\tau_{n+1}=\delta^{-1}(D+\delta)\left(\tau_{n+1}-\tau_{n}\right),
$$

and use the fact that the operation $\delta^{-1}(D+\delta)$ increases degree to see that this iterative solution converges as $n \rightarrow \infty$ in each degree. To show that (6-1) has a unique solution, take another solution $\tilde{\tau}$ and subtract to get

$$
\tilde{\tau}-\tau=\delta^{-1}(D+\delta)(\tilde{\tau}-\tau) .
$$

By counting degrees again, we see that $\tilde{\tau}=\tau$. Next we have to show that if $\tau$ is a solution of (6-1), then $D \tau=0$. As $\delta^{-1} \delta^{-1}=0$, applying $\delta^{-1}$ to (6-1) gives

$$
\delta^{-1} \tau=\delta^{-1} \eta
$$


Then we have, as $\delta \delta^{-1}+\delta^{-1} \delta$ is the identity on $C^{\infty}\left(W \otimes \Lambda^{q}\right)$,

$$
\begin{aligned}
\delta^{-1} D \tau & =\delta^{-1}(D+\delta) \tau-\delta^{-1} \delta \tau=\tau-\eta-\delta^{-1} \delta \tau \\
& =\delta \delta^{-1} \tau-\eta=\delta \delta^{-1} \eta-\eta=-\delta^{-1} \delta \eta=0 .
\end{aligned}
$$

Then, remembering that $D(D \tau)=0$,

$$
D \tau=\left(\delta^{-1} \delta+\delta \delta^{-1}\right) D \tau=\delta^{-1} \delta D \tau=\delta^{-1}(D+\delta) D \tau .
$$

By the previous degree argument, this implies that $D \tau=0$, and concludes showing that the back map is well defined.

To show that the composition one way round is the identity, apply $\delta \delta^{-1}$ to $(6-1)$ :

$$
\delta \delta^{-1} \tau=\delta \delta^{-1} \eta=\left(\delta^{-1} \delta+\delta \delta^{-1}\right) \eta=\eta .
$$

Finally we show that the composition of the maps the other way round is also the identity. Begin with $\tau$ satisfying $D \tau=0$. Then, as required

$$
\tau=\left(\delta^{-1} \delta+\delta \delta^{-1}\right) \tau=\delta^{-1}(D+\delta) \tau+\delta \delta^{-1} \tau .
$$

Now the classical $q$-forms do give rise to flat Weyl $q$-forms, as $C^{\infty}(M) \otimes \Lambda^{q}$ is contained in the kernel of $\delta$, so we can use (6-1) to find a corresponding flat section. However the kernel of $\delta$ in $C^{\infty}\left(W \otimes \Lambda^{q}\right)$ is much larger than the classical $q$-forms. If we take the wedge product of two flat sections coming from classical $q$-forms, we get a flat section, but not necessarily one coming from a classical $q$-form.

We are then faced with a choice: either to stick with the associative framework given by flat Weyl forms and sacrifice correspondence with the classical forms, or to try to maintain the correspondence but accept that associativity will only be approximately true.

Remember that a function $f \in C^{\infty}(M)$ has a corresponding quantisation beginning $f+y^{k} \nabla_{k} f+\frac{1}{2} y^{k} y^{j} \nabla_{k} \nabla_{j} f+\cdots$, so if we had a 1 -form also expanded in the form $\eta+y^{j} \eta_{[j]}+\cdots$, we would get

$$
\left[f+y^{i} \nabla_{i} f+\cdots, \eta+y^{i} \eta_{[i]}+\cdots\right]=-i \hbar \omega^{k j} \eta_{[j]} \nabla_{k} f+\cdots,
$$

so (using $-i \hbar$ instead of $\hbar$ to fit the usual notation of the Fedosov theory), we would have to have the quantisation of the 1 -form beginning $\eta+y^{k} \nabla_{k} \eta+\cdots$. However a calculation of the leading order expansion of the flat 1-form whose lowest order part is $\eta$ gives $\eta_{n}+\frac{1}{2} y^{k}\left(\eta_{n ; k}-\eta_{k ; n}\right)+\cdots$. We conclude that taking flat 1 -forms is really not the right thing to do. Also note that the supercommutator of two 1-forms is, to leading order,

$$
\left[\eta+y^{j} \nabla_{j} \eta+\cdots, \xi+y^{k} \nabla_{k} \xi+\cdots\right]=-i \hbar \omega^{j k} \nabla_{j} \eta \wedge \nabla_{k} \xi,
$$

also as previously described. 


\section{References}

[Beggs 2003] E. J. Beggs, "Braiding and exponentiating noncommutative vector fields", preprint, University of Swansea, 2003. math.QA/0306094

[Brzeziński 1993] T. Brzeziński, "Remarks on bicovariant differential calculi and exterior Hopf algebras", Lett. Math. Phys. 27:4 (1993), 287-300. MR 94g:58016 Zbl 0782.17009

[Connes 1994] A. Connes, Noncommutative geometry, Academic Press, San Diego, CA, 1994. MR 95j:46063 Zbl 0818.46076

[Drinfeld 1987] V. G. Drinfel'd, “Quantum groups”, pp. 798-820 in Proceedings of the International Congress of Mathematicians (Berkeley, 1986), vol. 2, edited by A. Gleason, Amer. Math. Soc., Providence, RI, 1987. MR 89f:17017 Zbl 0667.16003

[Drinfeld 1989] V. G. Drinfel'd, "Almost cocommutative Hopf algebras", Algebra i Analiz 1:2 (1989), 30-46. In Russian; translated in Leningrad Math. J. 1 (1990), 321-342. MR 91b:16046 Zbl 0718.16035

[Fedosov 1996] B. Fedosov, Deformation quantization and index theory, Mathematical Topics 9 , Akademie Verlag, Berlin, 1996. MR 97a:58179 Zbl 0867.58061

[Gelfand et al. 1998] I. Gelfand, V. Retakh, and M. Shubin, "Fedosov manifolds", Adv. Math. 136:1 (1998), 104-140. MR 99d:53023 Zbl 0945.53047

[Gomez and Majid 2003] X. Gomez and S. Majid, "Braided Lie algebras and bicovariant differential calculi over co-quasitriangular Hopf algebras", J. Algebra 261 (2003), 334-388. MR 2004h:17012 Zbl 1018.17009

[Hawkins 2004] E. Hawkins, "Noncommutative rigidity", Comm. Math. Phys. 246:2 (2004), 211235. MR 2005b:58011 Zbl 1055.58001

[Kosmann-Schwarzbach 1992] Y. Kosmann-Schwarzbach, "Jacobian quasi-bialgebras and quasiPoisson Lie groups", pp. 459-489 in Mathematical aspects of classical field theory (Seattle, WA, 1991), edited by M. J. Gotay et al., Contemp. Math. 132, Amer. Math. Soc., Providence, RI, 1992. MR 94b:17025 Zbl 0847.17020

[Kostant 1963] B. Kostant, "Lie group representations on polynomial rings", Amer. J. Math. 85 (1963), 327-404. MR 28 \#1252 Zbl 0124.26802

[Madore 1999] J. Madore, An introduction to noncommutative differential geometry and its physical applications, 2nd ed., London Math. Soc. Lecture Note Series 257, Cambridge University Press, Cambridge, 1999. MR 2000k:58008 Zbl 0942.58014

[Majid 1995] S. Majid, Foundations of quantum group theory, Cambridge University Press, Cambridge, 1995. MR 97g:17016 Zbl 0857.17009

[Majid 1998a] S. Majid, "Classification of bicovariant differential calculi", J. Geom. Phys. 25:1-2 (1998), 119-140. MR 99a:58013 Zbl 0952.17012

[Majid 1998b] S. Majid, "Quantum double for quasi-Hopf algebras”, Lett. Math. Phys. 45:1 (1998), 1-9. MR 2000b:16077 Zbl 0940.16018

[Majid and Oeckl 1999] S. Majid and R. Oeckl, "Twisting of quantum differentials and the Planck scale Hopf algebra”, Comm. Math. Phys. 205 (1999), 617-655. MR 2000h:58018 Zbl 0939.58007

[Woronowicz 1989] S. L. Woronowicz, "Differential calculus on compact matrix pseudogroups (quantum groups)”, Comm. Math. Phys. 122:1 (1989), 125-170. MR 90g:58010 Zbl 0751.58042 
EDWIN J. BEGGS

DEPARTMENT OF MATHEMATICS

UNIVERSITY OF WALES

SWANSEA, SA2 8PP

UNITED KINGDOM

E.J.Beggs@Swansea.ac.uk

\section{SHAHN MAJID}

SCHOOL OF Mathematical SCIENCES

QueEn Mary College

UNIVERSITY OF LONDON

327 MILE END RoAD

LONDON E1 4NS

UNITED KINGDOM

s.majid@qmul.ac.uk 